\title{
On the Codes Related to the Higman-Sims Graph
}

\author{
Wolfgang Knapp \\ Mathematisches Institut \\ Universität Tübingen, Germany \\ wolfgang . knapp@uni-tuebingen.de
}

\author{
Hans-Jörg Schaeffer \\ Mathematisches Institut \\ Universität Tübingen, Germany \\ hjs@hjschaeffer.de
}

Submitted: Apr 10, 2014; Accepted: Jan 10, 2015; Published: Jan 27, 2015

Mathematics Subject Classifications: 94B25 94B27 20B25 20D08 05C25

\begin{abstract}
All linear codes of length 100 over a field $F$ which admit the Higman-Sims simple group HS in its rank 3 representation are determined. By group representation theory it is proved that they can all be understood as submodules of the permutation module $F \Omega$ where $\Omega$ denotes the vertex set of the Higman-Sims graph. This module is semisimple if $\operatorname{char} F \neq 2,5$ and absolutely indecomposable otherwise. Also if $\operatorname{char} F \in\{2,5\}$ the submodule lattice is determined explicitly. The binary case $F=\mathbb{F}_{2}$ is studied in detail under coding theoretic aspects. The HS-orbits in the subcodes of dimension $\leqslant 23$ are computed explicitly and so also the weight enumerators are obtained. The weight enumerators of the dual codes are determined by MacWilliams transformation. Two fundamental methods are used: Let $v$ be the endomorphism determined by an adjacency matrix. Then in $H_{22}=\operatorname{Im} v$ the HS-orbits are determined as $v$-images of HS-orbits of certain low weight vectors in $F \Omega$ which carry some special graph configurations. The second method consists in using the fact that $H_{23} / H_{21}$ is a Klein four group under addition, if $H_{23}$ denotes the code generated by $H_{22}$ and a "Higman vector" $x(m)$ of weight 50 associated to a heptad $m$ in the shortened Golay code $G_{22}$, and $H_{21}$ denotes the doubly even subcode of $H_{22} \leqslant H_{78}=H_{22}{ }^{\perp}$. Using the mentioned observation about $H_{23} / H_{21}$ and the results on the HS-orbits in $H_{23}$ a model of G. Higman's geometry is constructed, which leads to a direct geometric proof that G. Higman's simple group is isomorphic to HS. Finally, it is shown that almost all maximal subgroups of the Higman-Sims group can be understood as stabilizers in HS of code words in $H_{23}$.
\end{abstract}

Keywords: Higman-Sims simple group, rank 3 representation, graph, linear code, Hamming weight, Higman's geometry

\section{Introduction}

In [20] a systematic program to determine all codes admitting a prescribed permutation group $G$ has been proposed. Hitherto in many cases good codes have been obtained from 
doubly-transitive permutation groups. In this paper the program of [20] is carried out for $G=$ HS, the simple group of Higman-Sims in its rank 3 permutation representation of degree 100. It turns out that any code over a field $F$ admitting $G$ can be obtained as a submodule of the permutation module $F \Omega$ over $F$, considering $\Omega$ as the ambient basis.

The binary codes are of particular interest. There is exactly one $G$-invariant subcode $H_{23}$ of dimension 23 over $\mathbb{F}_{2}$ which may be viewed as an amalgamation of 100 copies of the (shortened) binary Golay code of length 22. Let $v$ be the endomorphism determined by an adjacency matrix and let $H_{22}=\operatorname{Im} v$. Then $H_{22} \leqslant H_{78}=H_{22}{ }^{\perp}$. Let $H_{21}$ denote the doubly even subcode of $H_{22}$. Then $H_{22} \leqslant H_{23}$ and $H_{23} / H_{21}$ is a Klein four group under addition. The $G$-orbits on the weight classes of the codes of dimension at most 23 (which are all contained in $H_{23}$ ) are determined by elementary algebraic and combinatorial arguments. The two $G$-invariant subcodes $H_{22}^{\prime}$ and $H_{22}^{\prime \prime}$ of dimension 22 containing $H_{21}$ and different from $H_{22}$ are related to the $G$-orbits on the set of "Higman vectors" $x(m)$ of weight 50 associated to heptads $m$ in the shortened Golay code $G_{22}$. Two fundamental methods are used: the $G$-orbits in $H_{22}$ are determined as $v$-images of $G$-orbits of certain low weight vectors in $F \Omega$ which carry some special graph configurations. The second method consists in using the mentioned fact that $H_{23} / H_{21}$ is a Klein four group under addition. Also the natural action of the automorphism group $\bar{G}$ on $H_{23} / H_{21}$ is considered. Now, using also MacWilliams transformation, the weight enumerators for all non-trivial $G$-invariant binary subcodes are computed; some of them have rather good error correction capacity.

It turns out that the weight structure of $H_{23}$ is highly relevant for the combinatorial properties of the Higman-Sims graph. The structure of the subcodes of $H_{23}$ allows also the construction of a model of G. Higman's geometry [17] admitting an action of $G=\mathrm{HS}$. Thereby an isomorphism of HS and G. Higman's simple group is obtained. $H_{23}$ may also be used to describe a large part of the subgroup structure of HS in a similar way as it has been done for the Mathieu group $M_{24}$ and the (extended) binary Golay code of length 24 by Conway and Curtis. One can check that the parameters of several HS-invariant binary codes meet the Gilbert-Varšamov bound, see also [5].

The methods developed in this paper are not restricted to the case of the Higman-Sims simple group. In particular, one may obtain codes of length 77,56,50 and 16 from rank 3 groups corresponding to strongly regular subgraphs of the Higman-Sims graph. Note that N. Loebich has shown in [21] that $\mathcal{G}_{100}$ contains every known strongly regular graph having a vertex transitive group of automorphisms and no triangles as a subgraph.

\section{Remark}

This paper is based on talks of the first author in a research seminar in 1980 at the university of Tübingen and was completed essentially in 1982, in the time before $\mathrm{T}_{\mathrm{E}} \mathrm{X}$ or LTEX were available to the authors and before the Group ATLAS [1] and the Atlas of Brauer Characters [2] appeared. Due to its considerable length it could not be published at that time. Now, many years later, the first author found the time to rewrite the paper in $\mathrm{AT}_{\mathrm{E} X}$, so also making an electronic version available. Only a few changes of the original 
text were made, in particular some more recent references (e.g. to the ATLASes and to GAP) were added. It is clear that nowadays many of the results can be obtained easily just by computing using computer algebra systems like GAP (with packages GRAPE and GUAVA) or Sage. Note that in the present work only the MacWilliams transformations of several code enumerators were carried out by computer; all other results are obtained by arguments and computation by hand. It should be acknowledged that also some more recent research publications have some overlap with this paper, in particular V.D. Tonchev's paper [37]. Moreover, the code $H_{22}$ represents a particular instance of more recent general investigations, e.g. by Brouwer and Eijl [4] and Haemers, Peeters and van Ruckevorsel [12].

We thank the referees for some valuable suggestions.

\section{$1 \quad$ Background and preliminary results}

In this paper sets and groups are assumed finite, unless specified otherwise. If $\Omega$ is a set, then $\mathcal{P}(\Omega)$ denotes the set of all subsets of $\Omega ; \operatorname{Sym}_{\Omega}$ and $\mathrm{Alt}_{\Omega}$ denote the alternating and symmetric group of the set $\Omega, A_{n}$ and $\Sigma_{n}$ the alternating and symmetric group on $n$ letters. If $A$ and $B$ are groups then $A$ wr $B$ denotes the regular wreath product of order $|B|^{|A|}|A| . \quad A{ }^{k} \wedge B$ denotes a subdirect product of $A$ and $B$ where a factor group of order $k$ is identified. $M_{24-i}$ and $M_{12-i}$ denote the Mathieu groups of degree $24-i$ resp. $12-i$. $E_{p^{f}}$ denotes an elementary abelian group of order $p^{f}$.

Actions of a group $G$ onto a set $\Omega$ are right actions denoted by $(\alpha, g) \mapsto \alpha g$. If $G$ acts on $\Omega$ we write $(G, \Omega)$ for this action and - following a suggestion of H.Wielandt - we denote the set of $G$-orbits on $\Omega$ by $\Omega: G$. If $H \leqslant G$ then $G: H=\{H x \mid x \in G\}$ and $G:(H, H)=\{H x H \mid x \in G\}$.

$\mathbb{F}_{q}$ denotes a finite field of order $q$. Modules are always assumed to be finitely generated. If $R$ is a commutative ring with 1 and $M$ a free $R$-module then $\operatorname{rk}_{R} M$ denotes the $R$-rank of $M$. If $F$ is a field and $G$ a group then $F G$ denotes the group algebra of $G$ over $F$. If $U$ is an $F G$-module then $U^{*}$ denotes the dual $F G$-module. $U={ }_{F G} X \oplus Y$ denotes a direct decomposition of the $F G$-module $U$ into $F G$-submodules $X$ and $Y$. $J(A)$ denotes the Jacobson radical of an algebra $A$.

All other general notations are standard.

We shall need an elementary result concerning module reductions whose idea of proof is taken from Thompson [36, Theorem 1].

\section{(1.1) Proposition.}

Let $R$ be a principal ideal domain, $K$ its field of fractions, $P$ a maximal ideal of $R$ and $k=R / P$ its residue class field. Let $G$ be a finite group and $V$ an RG-lattice (i.e. a finitely generated $R$-free $R G$-module). Suppose $K \otimes_{R} V={ }_{K G} X \oplus Y$ is a direct sum of $K G$-submodules where $\operatorname{dim}_{K} X=s$. Then the following hold:

(1) $W=V \cap X$ is an $R G$-submodule and a pure $R$-submodule of $X$.

(2) $\operatorname{rk}_{R} V / W=\operatorname{dim}_{K} Y=\operatorname{rk}_{R} V-s$. 
(3) $k \otimes_{R} W$ is a $k G$-submodule of $k \otimes_{R} V$ of dimension $s$.

Proof. Clearly $W$ and $V$ are $R G$-submodules of $K \otimes_{R} V$. Since $V / W \cong(V+X) / X$ is isomorphic to an $R G$-submodule of $Y$ we see that $V / W$ is torsion free, hence $W$ is a pure submodule of $V$. Let $t=\operatorname{rk}_{R} V$. Then, of course, $\operatorname{rk}_{R} V / W=\operatorname{dim}_{K} Y=t-a$. By the theorem of elementary divisors there exists an $R$-basis $\left(b_{i}\right)_{1 \leqslant i \leqslant t}$ of $V$ and $\left(a_{i}\right)_{1 \leqslant i \leqslant t} \in R^{t}$ such that $a_{i} \mid a_{i+1}$ for $1 \leqslant i \leqslant t-1$ and $\left(a_{i} b_{i}\right)_{1 \leqslant i \leqslant t}$ is an $R$-basis of $W$. The fact that $W$ is a pure submodule entails that we may choose $a_{i}=1$ for $1 \leqslant i \leqslant t-s$ and $a_{i}=0$ for $t-s+1 \leqslant i \leqslant t$. Now it is obvious that $k \otimes_{R} W$ is an $s$-dimensional subspace of $k \otimes_{R} V$.

Proposition (1.1) is applied in this paper in the case $R=\mathbb{Z}, K=\mathbb{Q}, P=p \mathbb{Z}, k=\mathbb{F}_{p}$ and $V=\mathbb{Z} \Omega$, the integral permutation module of a group $G$ acting (transitively) on a set $\Omega$. In this case $\mathbb{Q} \otimes_{\mathbb{Z}} V=\mathbb{Q} \Omega$ and $\mathbb{F}_{p} \otimes_{\mathbb{Z}} V=\mathbb{F}_{p} \Omega$ are viewed in the obvious way as the permutation modules over $\mathbb{Q}$ resp. $\mathbb{F}_{p}$ defined by the same action of $G$ on $\Omega$.

\section{(1.2) Lemma.}

Let $G$ be a group and let $V$ be an $F G$-module over a finite field $F$ with dual module $V^{*}$. Let $u_{1} V$ and $u_{1} V^{*}$ denote the set of all 1-dimensional subspaces of $V$ and $V^{*}$, respectively. Then the following hold:

(1) $\left|u_{1} V^{*}: G\right|=\left|u_{1} V: G\right|$,

(2) If $F=\mathbb{F}_{2}$ then $\left|V^{*}: G\right|=|V: G|$.

Proof. Obviously, (2) is a consequence of (1). The natural $G$-invariant pairing $\phi: V \times$ $V^{*} \rightarrow F:(x, y) \mapsto x y$ may be used to show that $\left(u_{1} V, u_{1} V^{*},\{(F x, F y) \mid x y=0\}\right)$ is a projective space (with the hyperplanes in the rôle of blocks) admitting $G$ as a group of automorphisms. Brauer's Lemma ([3, p.933 f]) or the theorem of Dembowski-HughesParker $([9, \mathrm{p} .81])$ shows that $G$ has on $u_{1} V$ and on $u_{1} V^{*}$ the same permutation character, so (1) easily follows.

We shall use the following concepts from coding theory.

An ordered pair $(V, B)$, where $V$ is a finitely generated free $F$-module over a commutative ring $F$ and $B$ is an $F$-basis of $V$, is called a Hamming space over $F$. In this paper $F$ will always be a field. $\operatorname{dim}_{F} V$ is called the length of the Hamming space $(V, B)$. A Hamming space $(V, B)$ carries the following canonical structures.

Let $B=\left(e_{i}\right)$ and let $x=\sum x_{i} e_{i}$ for any $x \in V$.

(i) $V$ carries the nondegenerate symmetric bilinear form $(x, y) \mapsto\langle x, y\rangle=\langle x, y\rangle_{B}=$ $\sum x_{i} y_{i}$.

(ii) $V$ is a commutative and associative $F$-algebra with respect to the coordinatewise multiplication $(x, y) \mapsto \sum\left(x_{i} y_{i}\right) e_{i}$. 
(iii) $V$ carries the norm $w_{B}: x \mapsto w(x)=w_{B}(x):=\sum\left|x_{i}\right|$ where $|\cdot|$ denotes the trivial absolute value of $F . w(x)=w_{B}(x)$ is called the (Hamming) weight of $x$. Let $\operatorname{supp}(x)=\operatorname{supp}_{B}(x)=\left\{e_{i} \mid x_{i} \neq 0\right\}$ denote the support of $x$ with respect to $B$. Then, of course, $w(x)=|\operatorname{supp}(x)|$. To the norm $w_{B}$ there corresponds canonically the Hamming metric $d_{B}$ defined by $d_{B}(x, y):=w_{B}(x-y)$.

For any subset $X$ of $V$ let $W_{i}(X)$ denote the set of all vectors in $X$ of weight $i$. Furthermore let $X^{\perp}$ denote the set of all vectors in $V$ orthogonal to every element of $X$ with respect to $\langle\cdot, \cdot\rangle$. Of course, $X^{\perp}=\langle X\rangle^{\perp}$ is a (linear) subspace of $V$ of dimension $\operatorname{dim} V-\operatorname{dim}\langle X\rangle$.

Any triple $(V, B, C)$ where $C$ is a subspace of $V$ is called a linear code having ambient space $V$ and ambient basis $B$. If the Hamming space $(V, B)$ is given by the context we usually write $C=(V, B, C)$. $C$ is said to be an $(n, k)$-code if $\operatorname{dim} V=n$ and $\operatorname{dim} C=k$. Throughout the paper we shall follow the convention that a "code" is always understood to be a linear code.

If $C$ is a code of length $n$, the $(n+1)$-tuple $\left(\left|W_{i}(C)\right|\right)_{0 \leqslant i \leqslant n}$ is called the weight distribution of $C$, and the homogeneous polynomial $\sum\left|W_{i}(C)\right| \xi^{i} \eta^{n-i} \in \mathbb{C}[\xi, \eta]$ of degree $n$ is called the weight enumerator of $C$. The weight enumerators of a code $C$ and of its "dual" $C^{\perp}$ determine each other via the MacWilliams identities, see e.g. [16].

A morphism $(V, B, C) \rightarrow\left(V^{\prime}, B^{\prime}, C^{\prime}\right)$ of codes over $F$ is by definition an injective $F$ linear map $\mu: V \rightarrow V^{\prime}$ with $C \mu \subseteq C^{\prime}$ sending any $e \in B$ to a scalar multiple of some $e^{\prime} \in B^{\prime}$. The codes $C$ and $C^{\prime}$ are isomorphic if $\mu$ is bijective and $C \mu=C^{\prime}$.

$\operatorname{ML}(C)$ denotes the group of all (code) automorphisms from $(V, B, C)$ to itself, the monomial linear group of $C$. $(\mathrm{ML}(C)$ can be represented by monomial matrices, with respect to the ambient basis $B$.) Let $B=\left(e_{i}\right)_{i \in \Omega}$. Every $\mu \in \operatorname{ML}(C)$ determines a permutation $\bar{\mu}$ of $\Omega$ by $e_{i} \mu \in\left\langle e_{\bar{\mu}}\right\rangle=F e_{\bar{\mu}}$. The map $\mu \mapsto \bar{\mu}$ is an epimorphism of $\operatorname{ML}(C)$ onto a subgroup $P \operatorname{ML}(C)$ of the symmetric group $\operatorname{Sym}_{\Omega}$. We call $\operatorname{PML}(C)$ the permutation group of $C$. The code $C$ is said to admit a permutation group $G$ acting on $\Omega$ if $G$ is a subgroup of $\operatorname{PML}(C)$. The elements of $\operatorname{ker}(\mu \mapsto \bar{\mu})$ form the group $\operatorname{DL}(C)$ of diagonal automorphisms of $C$.

In the following we fix a $3-(22,6,1)$-design $\mathcal{W}_{22}=\left(P_{22}, B_{22}, I_{22}\right)$. It is a result of Witt $[39,40]$ that such a design exists and is unique up to isomorphism. $\mathcal{W}_{22}$ may be viewed as an extension of the projective plane $\mathrm{PG}(2,4)=\left(P_{21}, B_{21}, I_{21}\right)$ where $P_{22}=P_{21} \cup\{\infty\}$ and $B_{22}$ is the union of $B_{21}^{\prime}=\left\{\{\infty\} \cup g \mid g \in B_{21}\right\}$ and an orbit $\mathcal{H}_{1}$ of $\operatorname{PSL}(3,4)$ on the set of hyperovals of $\mathrm{PG}(2,4)$, see [15]. The full group of automorphisms is the automorphism group $\operatorname{Aut}\left(M_{22}\right)=\overline{M_{22}}$ of the Mathieu simple group $M_{22}$ of order 443,520 where $\left|\overline{M_{22}}: M_{22}\right|=2$. The permutation group $M_{22}$ acting on $P_{22}$ is a transitive extension of $M_{21}=\operatorname{PSL}(3,4)$.

The (shortened) binary Golay code of length 22 may be described in terms of $\mathcal{W}_{22}$ as follows, see also $[20,5.3]$. Let $V=\mathbb{F}_{2} P_{22}$ be the $\mathbb{F}_{2}$-vector space with basis $P_{22}$. Then $\left(V, P_{22}\right)$ is a Hamming space. Let $G_{11}$ be the subspace of $V$ generated by all characteristic functions of blocks of $\mathcal{W}_{22}$, i.e. $G_{11}=\left\langle b^{\bullet} \mid b \in B_{22}\right\rangle$ where $b^{\bullet}=\sum\left\{\delta(b, x) x \mid x \in P_{22}\right\}$ and $\delta(b, x)=0$ if $x x_{22} b, \delta(b, x)=1$ if $x \mathrm{I}_{22} b$. 
It is easily seen that $G_{11}$ is a $(22,11)$-code which is self-dual, i.e. $G_{11}^{\perp}=G_{11}$. Moreover $G_{11}=\left\langle\mathbb{1}_{22}\right\rangle \oplus G_{10}$ where $\mathbb{1}_{22}=\sum P_{22}$ and $G_{10}=\left\langle b^{\bullet}+c^{\bullet} \mid\{b, c\} \subseteq B_{22}\right\rangle=\left\langle\mathbb{1}_{22}+b^{\bullet}\right| b \in$ $\left.B_{22}\right\rangle$.

Let $G_{12}=G_{10}^{\perp}$. Then $G_{12}$ is called the (shortened) Golay code of length 22. Of course, $G_{12}$ is a $(22,12)$-code containig $G_{11}$. There are two more codes $G_{11}^{\prime}$ and $G_{11}^{\prime \prime}$ properly between $G_{10}$ and $G_{12}$. The following assertions about the binary Golay code of length 22 are well known and easy to prove.

\section{(1.3) Proposition.}

$G_{12}$ and its subcodes have the following properties.

(1) $\operatorname{ML}\left(G_{12}\right)=\operatorname{ML}\left(G_{11}\right)=\operatorname{ML}\left(G_{10}\right)=\overline{M_{22}}$.

(2) $\operatorname{ML}\left(G_{11}^{\prime}\right)=\operatorname{ML}\left(G_{11}^{\prime \prime}\right)=M_{22}$, and $\overline{M_{22}}$ interchanges $G_{11}^{\prime}$ and $G_{11}^{\prime \prime} \cdot\left(G_{11}^{\prime}\right)^{\perp}=G_{11}^{\prime \prime}$.

(3) $M_{22}$ acts (as a linear group) irreducibly on $G_{10}$ and trivially on $G_{12} / G_{10}$.

(4) The weight distributions of the codes are as follows:

\begin{tabular}{|c|c|c|c|c|}
\hline$i$ & $\left|W_{i}\left(G_{10}\right)\right|$ & $\left|W_{i}\left(G_{11}\right)\right|$ & $\left|W_{i}\left(G_{11}^{\prime}\right)\right|$ & $\left|W_{i}\left(G_{12}\right)\right|$ \\
\hline 0 & 1 & 1 & 1 & 1 \\
\hline 6 & - & 77 & - & 77 \\
\hline 7 & - & - & 176 & 352 \\
\hline 8 & 330 & 330 & 330 & 330 \\
\hline 10 & - & 616 & - & 616 \\
\hline 11 & - & - & 672 & 1,344 \\
\hline 12 & 616 & 616 & 616 & 616 \\
\hline 14 & - & 330 & - & 330 \\
\hline 15 & - & - & 176 & 352 \\
\hline 16 & 77 & 77 & 77 & 77 \\
\hline 22 & - & 1 & - & 1 \\
\hline
\end{tabular}

In the table only occurring weights $i$ are displayed; - is printed in place of 0.

Note that $\left|W_{i}\left(G_{11}^{\prime}\right)\right|=\left|W_{i}\left(G_{11}^{\prime \prime}\right)\right|$, since $G_{11}^{\prime}$ and $G_{11}^{\prime \prime}$ are conjugate under $\overline{M_{22}}$.

Moreover,

$$
\begin{aligned}
& G_{11}=\left\{x \in G_{12} \mid w(x) \equiv 0 \quad(\bmod 2)\right\} \\
& G_{10}=\left\{x \in G_{12} \mid w(x) \equiv 0 \quad(\bmod 4)\right\}
\end{aligned}
$$

(5) $M_{22}$ acts transitively on each nonempty $W_{i}\left(G_{12}\right), i \neq 7,11,15$.

$W_{i}\left(G_{12}\right), i \in\{7,11,15\}$, splits into two $M_{22}$-orbits $W_{i}\left(G_{11}^{\prime}\right)$ and $W_{i}\left(G_{11}^{\prime \prime}\right)$ which are fused under $\overline{M_{22}}$.

$\overline{M_{22}}$ acts transitively on every nonempty $W_{i}\left(G_{12}\right)$. 
(6) Let $x \in W_{i}\left(G_{12}\right), 0 \neq i \neq 22$. Then the stabilizer subgroup $\left(M_{22}\right)_{x}$ is a maximal subgroup of $M_{22}$ having the following structure:

\begin{tabular}{|c|c|}
\hline$i$ & structure of $\left(M_{22}\right)_{x}$ \\
\hline $6 / 16$ & $E_{16} A_{6}$ \\
\hline $7 / 15$ & $A_{7}$ \\
\hline $8 / 14$ & $E_{8} \mathrm{GL}(3,2)$ \\
\hline $10 / 12$ & $M_{10}$ \\
\hline 11 & $\operatorname{PSL}(2,11)$ \\
\hline
\end{tabular}

In each case $\left(M_{22}\right)_{x}$ acts transitively on $\operatorname{supp}(x)$. There are exactly two conjugacy classes in $M_{22}$ of such stabilizers for $i \in\{7,11,15\}$ which are fused under $\operatorname{Aut}\left(M_{22}\right) \cong \overline{M_{22}}$.

Remark. Of course, $\left(M_{22}\right)_{x}=\left(M_{22}\right)_{x+\mathbb{1}_{22}}$. There is exactly one more conjugacy class of maximal subgroups of $M_{22}$, namely the stabilizers in $M_{22}$ of 2 -subsets of $P_{22}$. These maximal subgroups, of index 231, are isomorphic to $E_{16} \Sigma_{5}$, see [7, Table 3].

It will be convenient to identify the vectors of $\mathbb{F}_{2} P_{22}$ with their supports in $P_{22}$. So $\mathbb{F}_{2} P_{22}$ may be viewed as the (power) set $\mathcal{P}\left(P_{22}\right)$ of all subsets of $P_{22}$. We recall the following notions.

\section{(1.4) Definition.}

The elements of $W_{i}\left(G_{12}\right)$, resp. their supports, are called hexads $(i=6)$, heptads $(i=7)$, octads $(i=8)$, decads $(i=10)$, endecads $(i=11)$ and dodecads $(i=12)$ of $\mathcal{W}_{22}$.

The hexads of the definition are the blocks of $\mathcal{W}_{22}$. For later use we set $\mathcal{M}^{\prime}=W_{7}\left(G_{11}^{\prime}\right)$ and $\mathcal{M}^{\prime \prime}=W_{7}\left(G_{11}^{\prime \prime}\right)$. Then $\mathcal{M}^{\prime}$ and $\mathcal{M}^{\prime \prime}$ are the orbits of $M_{22}$ in the set of heptads of $\mathcal{W}_{22}$.

We shall need some more detailed properties of $G_{12}$. A subset $T$ of $P_{22}$ is called independent if there is no hexad of $\mathcal{W}_{22}$ incident with every element of $T$, otherwise dependent. Furthermore, we introduce the block graph $\mathcal{G}_{77}=\left(B_{22}, \Delta_{77}\right)$ by taking the 77 blocks of $\mathcal{W}_{22}$ as vertices, joining two vertices by an edge in $\Delta_{77}$ if and only if they are disjoint. $\left(\mathcal{G}_{77}\right.$ is a strongly regular graph of valency 16 , having $\overline{M_{22}}$ in its action on the blocks of $\mathcal{W}_{22}$ as its group of automorphisms.)

\section{(1.5) Lemma.}

Let $B$ be a set of at most 5 blocks of $\mathcal{W}_{22}$ such that $\sum\left\{b^{\bullet} \mid b \in B\right\}=\mathbb{1}_{22}$. Then $|B|=5$ and exactly one of the following holds:

(i) There exists a 2-subset $T=\{x, y\}$ of $P_{22}$ such that $B$ is the set of all blocks incident with $x$ and $y . \mathcal{G}_{77}$ induces on $B$ the null graph.

(ii) There exists an independent 4-subset $T$ of $P_{22}$ such that $B$ is the union of the set $B_{0}$ of all blocks defined by the 4 triangles in $T$ and the unique block $b_{1}$ disjoint from $\bigcup\left\{b \mid b \in B_{0}\right\}$. $\mathcal{G}_{77}$ induces on $B$ a 4-claw. 
(iii) There exist two disjoint 2-subsets $T_{1}=\left\{x_{1}, y_{1}\right\}$ and $T_{2}=\left\{x_{2}, y_{2}\right\}$ such that $T_{1} \cup T_{2}$ is dependent and $B$ consists of the unique block $b_{0}$ determined by $T_{1} \cup T_{2}$ and of four blocks $b_{1}, b_{2}, b_{3}, b_{4}$ such that $b_{1}$ intersects $b_{2}$ in $T_{1}, b_{3}$ intersects $b_{4}$ in $T_{2}$ and $b_{1} \cup b_{2}$ is disjoint from $b_{3} \cup b_{4}$. $\mathcal{G}_{77}$ induces on $B$ a quadrangle and an isolated point.

Moreover, any block configuration $B$ described above has the property $\sum\left\{b^{\bullet} \mid b \in B\right\}=$ $\mathbb{1}_{22}$.

Proof. Assume $|B| \leqslant 4$. Then $|B|=4$ since $3 \cdot 6=18<22$. Let $B=\left\{b_{0}, b_{1}, b_{2}, b_{3}\right\}$. The $b_{i}$ are not pairwise disjoint. Without loss of generality we may suppose that $b_{1}, b_{2}$ and $b_{3}$ intersect in the point $\infty$ which implies that $b_{1}, b_{2}$ and $b_{3}$ correspond to lines of PG $(2,4)$ and $b_{0}$ corresponds to a hyperoval which immediately leads to a contradiction. Therefore we have $|B|=5$.

Let $M_{i}=\left\{x \mid x \in P_{22}\right.$ and $x$ is incident with exactly $i$ elements of $\left.B\right\}$ for $0 \leqslant i \leqslant 5$. We have the partition $P_{22}=M_{1} \uplus M_{3} \uplus M_{5}$.

If $\left|M_{5}\right| \geqslant 2$ then $\left|M_{5}\right|=2$ and (i) holds. $\left|M_{5}\right|=1$ is impossible, as is seen by considering the projective plane $\left(\mathcal{W}_{22}\right)_{x}$ where $M_{5}=\{x\}$.

If $M_{5}=\emptyset$ then we have $M_{3} \neq \emptyset$ since the blocks of $B$ cannot be pairwise disjoint. We may assume without loss of generality that $\infty \in M_{3}$. Let $B=\left\{b_{0}, b_{1}, b_{2}, b_{3}, b_{4}\right\}$ such that $\infty$ is incident with $\left\{b_{0}, b_{1}, b_{2}\right\}$. Then $b_{0}, b_{1}, b_{2}$ may be viewed as lines and $b_{3}, b_{4}$ as hyperovals in $\mathrm{PG}(2,4)$. If $b_{0}, b_{1}, b_{2}$ are confluent lines then it easily follows that (iii) holds. If $b_{0}, b_{1}, b_{2}$ are not confluent it follows that (ii) holds. Note that in both cases $\left|M_{3}\right|=4$ holds.

It is immediate that all configurations $B$ of types (i), (ii), (iii) have the required property $\sum\left\{b^{\bullet} \mid b \in B\right\}=\mathbb{1}_{22}$.

\section{(1.6) Lemma.}

Let $C$ be a set of blocks of $\mathcal{W}_{22}$ such that $|C|=4$ and $\sum\left\{b^{\bullet} \mid b \in C\right\}=0$. Set $M_{i}=\{x \mid$ $x \in P_{22}$ and $x$ is incident with exactly $i$ elements of $\left.C\right\}, m_{i}=\left|M_{i}\right|$ for $0 \leqslant i \leqslant 4$. Then $m_{0}=10$ and $m_{2}=12$.

Proof. Of course $m_{0}+m_{2}+m_{4}=22$. It is easy to derive a contradiction if $m_{4} \neq 0$. Therefore $m_{4}=0$ and we may assume without loss that $\infty \in M_{2}$. In this case $C$ consists of 2 lines and 2 hyperovals and the assertion is easily verified. (Note that $C$ cannot contain two disjoint blocks.)

\section{(1.7) Lemma.}

Let $C$ be a set of blocks of $\mathcal{W}_{22}$ such that $|C|=6$ and $\sum\left\{b^{\bullet} \mid b \in C\right\}=0$. Set $M_{i}=\{x \mid$ $x \in P_{22}$ and $x$ is incident with exactly $i$ elements of $\left.C\right\}, m_{i}=\left|M_{i}\right|$ for $0 \leqslant i \leqslant 6$.

Then exactly one of the following holds:

(i) Two elements of $C$ are disjoint.

(ii) $m_{6} \neq 0$.

(iii) $m_{0}=7, m_{2}=12$ and $m_{4}=3$. 
Proof. Suppose that $m_{6}=0$ and that no two elements of $C$ are disjoint. Let $b \in C$. The remaining 5 blocks in $C$ intersect $b$ in exactly 2 points. Counting incidences yields that $b$ contains exactly 2 elements of $M_{4}$ and 4 elements of $M_{2}$. Now it easily follows that $m_{4}=\left|M_{4}\right|=(6 \cdot 2) / 4=3$ and $m_{2}=(6 \cdot 4) / 2=12$. So (iii) holds.

Note that examples for the cases (ii) and (iii) in (1.7) easily can be constructed. In case (ii) $C$ is formed by a dual hyperoval in the projective plane $\operatorname{PG}(2,4)$ if $\infty \in M_{6}$.

\section{(1.8) Lemma.}

Let $C$ be a set of blocks of $\mathcal{W}_{22}$ such that $|C|=7$ and $\sum\left\{b^{\bullet} \mid b \in C\right\}=\mathbb{1}_{22}$. Set $M_{i}=\left\{x \mid x \in P_{22}\right.$ and $x$ is incident with exactly $i$ elements of $\left.C\right\}, m_{i}=\left|M_{i}\right|$ for $0 \leqslant i \leqslant 7$. Then either $m_{5}+m_{7}>0$ or $m_{1}=12$ and $m_{3}=10$.

Proof. Suppose $m_{5}+m_{7}=0$. Then $m_{1}+m_{3}=22$, and by counting incidences we obtain $42=7 \cdot 6=m_{1}+3 m_{3}$. It follows $m_{1}=12$ and $m_{3}=10$.

(1.9) Proposition. (1) The heptads of $\mathcal{W}_{22}$ are exactly the hyperblocks in the sense of Lüneburg [22, p.98].

(2) Recall that $V=\mathbb{F}_{2} P_{22}$. Let $y \in W_{7}(V)$. Then the following assertions are equivalent:

(a) y is a heptad.

(b) $\left\langle y, b^{\bullet}\right\rangle=1$ for all $b \in B_{22}$.

(c) $|\operatorname{supp}(y) \cap b| \in\{1,3\}$ for all $b \in B_{22}$.

(3) Let $y, z$ be heptads of $\mathcal{W}_{22}$. Then the following assertions are equivalent:

(a) $y$ and $z$ belong to the same $M_{22}$-orbit.

(b) $\langle y, z\rangle=1$.

(c) $|\operatorname{supp}(y) \cap \operatorname{supp}(z)| \in\{1,3,7\}$.

(4) Let $y, z$ be heptads of $\mathcal{W}_{22}$. Then the following assertions are equivalent:

(a) $y$ and $z$ belong to distinct $M_{22}$-orbits.

(b) $\langle y, z\rangle=0$.

(c) $|\operatorname{supp}(y) \cap \operatorname{supp}(z)| \in\{0,2,4\}$.

Proof. (2), (3) and (4) immediately follow from (1.3). (1) is then obvious.

For any $x \in V=\mathbb{F}_{2} P_{22}$ set $B_{i}(x)=\left\{b \in B_{22}|| \operatorname{supp}(x) \cap \operatorname{supp}(b) \mid=i\right\}, 0 \leqslant i \leqslant 6$.

\section{(1.10) Lemma.}

Let $y$ be a heptad of $\mathcal{W}_{22}$. Then the following hold.

(1) $B_{22}=B_{1}(y) \uplus B_{3}(y),\left|B_{1}(y)\right|=42$ and $\left|B_{3}(y)\right|=35$.

(2) $\left(M_{22}\right)_{y} \cong A_{7}$ has the orbits $B_{1}(y)$ and $B_{3}(y)$ in $B_{22}$. 
Proof. The assertion follows from (1.3) and (1.9), see also [22, 14.12].

\section{(1.11) Lemma.}

Let $y$ be a heptad of $\mathcal{W}_{22}, y \in \mathcal{M}^{\prime}$, say. For $0 \leqslant i \leqslant 7$ let $D_{i}(y)=\{z \mid z$ a heptad such that $|\operatorname{supp}(y) \cap \operatorname{supp}(z)|=i\}$. Then the following hold:

(1) If $D_{i}(y) \neq \emptyset$ then $\left(M_{22}\right)_{y} \cong A_{7}$ acts transitively on $D_{i}(y)$.

(2) $\mathcal{M}^{\prime}=D_{1}(y) \uplus D_{3}(y) \uplus D_{7}(y)$ and $\mathcal{M}^{\prime \prime}=D_{0}(y) \uplus D_{2}(y) \uplus D_{4}(y)$.

(3) The cardinalities of $D_{i}(y)$ are given in the following table:

\begin{tabular}{|c||c|c|c|c|c|c|}
\hline$i$ & 0 & 1 & 2 & 3 & 4 & 7 \\
\hline$\left|D_{i}(y)\right|$ & 15 & 70 & 126 & 105 & 35 & 1 \\
\hline
\end{tabular}

Proof. (3) may be obtained from the Leech triangle [7, p.226]. It is clear from the definition that the sets $D_{i}(y)$ are $\left(M_{22}\right)_{y}$-invariant. Inspection of the character table of $M_{22}$ and Frobenius reciprocity then yield (1) and (2).

We also need a result on endecads of $\mathcal{W}_{22}$.

\section{(1.12) Lemma.}

Let $e$ be an endecad of $\mathcal{W}_{22}, \bar{e}=e+\mathbb{1}_{22}$ its complement. Then the following hold.

(1) $\left|B_{1}(e)\right|=\left|B_{5}(e)\right|=11$ and $\left|B_{3}(e)\right|=55$.

(2) $B_{22}=B_{1}(e) \uplus B_{3}(e) \uplus B_{5}(e)$.

(3) $B_{3}(e)=B_{3}(\bar{e}), B_{1}(e)=B_{5}(\bar{e})$ and $B_{5}(e)=B_{1}(\bar{e})$.

(4) $\left(M_{22}\right)_{e} \cong \operatorname{PSL}(2,11)$ acts transitively on $\operatorname{supp}(e), \operatorname{supp}(\bar{e})$ and any nonempty $B_{i}(e)$.

Proof. (1) and (2) may be obtained from (1.3). (3) is obvious from the definition of the $B_{i}(x)$. (4) is verified by inspection. (Note that $\operatorname{PSL}(2,11)$ acts 3 -homogeneously on 11 points; this forces $\left(M_{22}\right)_{e}$ to act transitively on $B_{3}(e)$.)

We use the original definition of the Higman-Sims graph and group [16, 22] as follows.

Let $\alpha \notin P_{22} \uplus B_{22}$ and set $\Omega=\{\alpha\} \uplus P_{22} \uplus B_{22}$. Then $|\Omega|=1+22+77=100$. A graph $\mathcal{G}_{100}=(\Omega, E)$ with vertex set $\Omega$ is defined by

$$
\begin{aligned}
E & =\left\{\{\alpha, x\} \mid x \in P_{22}\right\} \\
& \uplus\left\{\{x, y\} \mid x \in P_{22}, y \in B_{22} \text { and }(x, y) \in \mathrm{I}_{22}\right\} \\
& \uplus \quad\left\{\{y, z\} \mid y, z \in B_{22} \text { and } y \text { disjoint from } z \text { in } \mathcal{W}_{22}\right\} .
\end{aligned}
$$


It is easily shown that $\mathcal{G}_{100}$ is a strongly regular graph with valency $k=22$ and intersection parameters $\lambda=0, \mu=6$ in D.G. Higman's sense [14]. $\mathcal{G}_{100}$ is called the Higman-Sims graph.

Let $\bar{G}=\operatorname{Aut}\left(\mathcal{G}_{100}\right)$. Then $\bar{G}$ contains a simple normal subgroup $G=$ HS of index 2 , the Higman-Sims simple group of order $44,352,000=2^{9} 3^{2} 5^{3} \cdot 7 \cdot 11 . \bar{G}$ acts as a rank 3 permutation group primitively on $\Omega$. Of course, $G_{\alpha} \cong M_{22}$ and $\bar{G}_{\alpha} \cong \bar{M}_{22}$. Moreover, $\bar{G} \cong \operatorname{Aut}(G)$.

We need some more detailed notation for the action of $G(\operatorname{resp} . \bar{G})$ on $\Omega$.

$G$ and $\bar{G}$ have the orbits $\Delta_{0}, \Delta_{1}, \Delta_{2}$ in $\Omega^{2}$ where

$$
\begin{aligned}
& \Delta_{0}=\{(\xi, \xi) \mid \xi \in \Omega\} \text { is the diagonal, } \\
& \Delta_{1}=\{(\xi, \eta) \mid\{\xi, \eta\} \in E\} \text { and } \\
& \Delta_{2}=\{(\xi, \eta) \mid \xi, \eta \in \Omega, \xi \neq \eta \text { and }\{\xi, \eta\} \notin E\} .
\end{aligned}
$$

We use the notation $\Delta_{i}(\xi)=\left\{\eta \mid(\xi, \eta) \in \Delta_{i}\right\}$ for the corresponding $G_{\xi}$-orbits. Of course, $\left|\Delta_{i}(\xi)\right|=1,22,77$ respectively.

The matrix $V_{i}$ in the centralizer algebra of $(G, \Omega)$ is defined by

$$
V_{i}=\left(f_{i}(\xi, \eta)\right)_{(\xi, \eta) \in \Omega^{2}}
$$

where $f_{i}(\xi, \eta)=1$ if $(\xi, \eta) \in \Delta_{i}$ and $f_{i}(\xi, \eta)=0$ otherwise $(i=0,1,2)$.

$V_{i}$ may be viewed as a matrix over any integral domain. If $F$ is a field and $F \Omega$ denotes the permutation module of $(G, \Omega)$ over $F$, to each $V_{i}$ there is naturally associated an endomorphism $v_{i}$ with the property

$$
\xi \mapsto \xi v_{i}=\sum f_{i}(\xi, \eta) \eta
$$

We use the convention $\Delta=\Delta_{1}$ and $v=v_{1}$ throughout the paper.

It is convenient to identify the $\mathbb{F}_{2}$ vector spaces $\mathcal{P}(\Omega)$ and $\mathbb{F}_{2} \Omega$ in the obvious way. We introduce special names for some interesting elements:

The elements $\Delta(\xi), \xi \in \Omega$, are called adjacency vectors of $\mathcal{G}_{100}$; arranged in a suitable way they form a (binary) adjacency matrix of $\mathcal{G}_{100}$.

$x \in \mathbb{F}_{2} \Omega$ is called a $\beta$-hexad for some $\beta \in \Omega$ if and only if there exists a $g \in G$ such that $\beta g=\alpha$ and $x g$ is a hexad of $\mathcal{W}_{22}$ (viewed as a subset of $P_{22}$ ). In the same way $\beta$-heptads, $\beta$-octads, $\beta$-decads, $\beta$-endecads and $\beta$-dodecads are defined.

If $m$ is an $\alpha$-heptad then the vector $x(m)=\alpha+m+B_{1}(m) \in W_{50}\left(\mathbb{F}_{2} \Omega\right)$ is called a Higman vector, see (1.10). (The terminus "Higman vector" refers to G. Higman's geometry and will be justified in Section 4.)

Note that if $x$ is a $\beta$-hexad and a $\gamma$-hexad then $\beta=\gamma$ or $\operatorname{supp}(x)=\Delta(\beta) \cap \Delta(\gamma)$; in the latter case $\{\beta, \gamma\}$ is uniquely determined by $x$. For $\beta$-heptads, $\beta$-octads, $\beta$-decads, $\beta$-endecads and $\beta$-dodecads $y$ the vertex $\beta$ is uniquely determined by $y$.

Let $U \leqslant \bar{G}=\operatorname{Aut}\left(\mathcal{G}_{100}\right)$ and let $\Omega: U=\left\{\Phi_{i} \mid 0 \leqslant i \leqslant t-1\right\}$. Then the matrix of the Higman-Sims graph with respect to $\left(\Phi_{i}\right)_{0 \leqslant i \leqslant t-1}$ (or less precisely with respect to $U$ ) is the matrix 


$$
A=\left(a_{i j}\right) \in \mathbb{Z}^{t \times t} \text { where } a_{i j}=\left|\Delta(\xi) \cap \Phi_{j}\right| \text { for } \xi \in \Phi_{i} .
$$

Note that $A$ is an adjacency matrix of $\mathcal{G}_{100}$ if $U=1$ and the transpose of an intersection matrix of $(\Omega, G)$ in D.G. Higman's sense [14] if $U=G_{\alpha}$.

\section{(1.13) Lemma.}

Let $m$ be an $\alpha$-heptad. Then $G_{\alpha, m}=G_{\alpha+m} \cong A_{7}$ and the $G_{\alpha, m}$-orbits in $\Omega$ are

$$
\left(\Psi_{i}\right)_{0 \leqslant i \leqslant 4}=\left(\{\alpha\}, m, B_{1}(m), \Delta(\alpha)+m, B_{3}(m)\right)
$$

with orbit lengths

$$
\left(\left|\Psi_{i}\right|\right)_{0 \leqslant i \leqslant 4}=(1,7,42,15,35) .
$$

The matrix of the Higman-Sims graph with respect to $\left(\Psi_{i}\right)$ is

$$
A=\left[\begin{array}{ccccc}
0 & 7 & 0 & 15 & 0 \\
1 & 0 & 6 & 0 & 15 \\
0 & 1 & 6 & 5 & 10 \\
1 & 0 & 14 & 0 & 7 \\
0 & 3 & 12 & 3 & 4
\end{array}\right]
$$

Proof. The assertion follows from (1.10) or [22, 14.12].

\section{(1.14) Proposition.}

Let $\mathbb{F}_{2} B_{22}$ be the permutation module over $\mathbb{F}_{2}$ for $M_{22}$ of dimension 77 given by the action on the blocks of $\mathcal{W}_{22}, \mathbb{1}_{77}=\sum B_{22}$. Then $\mathbb{F}_{2} B_{22}=\mathbb{F}_{2} M_{22}\left\langle\mathbb{1}_{77}\right\rangle \oplus Y$ where $Y=\left\langle\mathbb{1}_{77}\right\rangle^{\perp}$ is an indecomposable $\mathbb{F}_{2} M_{22}$-module of dimension 76 .

Proof. Since 77 is odd we have the decomposition $\mathbb{F}_{2} B_{22}=\left\langle\mathbb{1}_{77}\right\rangle \oplus Y$ where $Y=\left\langle\mathbb{1}_{77}\right\rangle^{\perp}$. Since $M_{22}$ acts as a rank 3 permutation group on $B_{22}$ we infer that either $Y$ is indecomposable or $E=\operatorname{End}_{\mathbb{F}_{2} M_{22}}\left(\mathbb{F}_{2} B_{22}\right)$ is semisimple. Now consider the intersection matrix of $M_{22}$ acting on $B_{22}$

$$
S=\left[\begin{array}{ccc}
0 & 1 & 0 \\
16 & 0 & 4 \\
0 & 15 & 12
\end{array}\right]
$$

(see [14] for example). It follows that $E$ contains a proper nilpotent element. Hence $E$ is not semisimple, therefore $Y$ is indecomposable.

\section{(1.15) Corollary.}

Let $\mathbb{F}_{2} \Omega$ be the permutation module over $\mathbb{F}_{2}$ for $G=\mathrm{HS}$ of dimension 100 given by the action on the vertices of $\mathcal{G}_{100}, \mathbb{1}=\sum \Omega$. Then $\langle\mathbb{1}\rangle^{\perp} /\langle\mathbb{1}\rangle=\mathbb{F}_{2} M_{22} Y \oplus Z$ where $Y$ and $Z$ are indecomposable $\mathbb{F}_{2} M_{22}$-modules of dimension 76 and 22 respectively.

Proof. Restriction of coordinates shows that $\langle\mathbb{1}\rangle^{\perp} \cong \mathbb{F}_{2} P_{22} \oplus \mathbb{F}_{2} B_{22}$ as an $\mathbb{F}_{2} M_{22}$-module. From (1.3) it follows that $\mathbb{F}_{2} P_{22}$ is indecomposable of dimension 22. Now (1.14) and the isomorphism theorems for modules yield the assertion. 
In an appendix to this section for the convenience of the reader two distinct adjacency matrices of the Higman-Sims graph $\mathcal{G}_{100}$ are displayed. We thank an anonymous referee for pointing out that an adjacency matrix for $\mathcal{G}_{100}$ is provided also by the graph database of Sage [34]. An adjacency matrix of $\mathcal{G}_{100}$ can also easily be computed directly by GAP using the Package AtlasRep. But such a matrix still would have to be transformed by additional programs to one of the shapes in Figure 1 or Figure 2 which visualize a better understanding of the graph structure.

In Figure 1 an adjacency matrix in a "canonical" form according to the definition of $\mathcal{G}_{100}$ is given. Here $\Omega$ is ordered according to a particular rank 3 stabilizer series: $\alpha$ is taken first, then two Singer-cycle orbits separated by a fixed-point follow. In this ordering of $\Omega$ the adjacency matrix displays the well known fact that $\mathcal{G}_{100}$ contains strongly regular subgraphs with vertex number 77 and valency 16 (the graph $\mathcal{G}_{77}$ mentioned above) and with vertex number 56 and valency 10 (called the "Gewirtz graph").

Detached at the bottom of the matrix a Higman vector $x(m)$ in the same ordering of $\Omega$ is printed.

In Figure 2 the ordering of $\Omega$ is such that the support of the given Higman vector $x(m)$ is taken first. The ordering is chosen also such that $\mathcal{G}_{100}$ induces on $\operatorname{supp}(x(m))$ and on its complement the "same" strongly regular graph of valency 7 (called the "HoffmanSingleton graph"). Figure 2 also obviously displays the $2 \cdot 50$ cocliques of size 15 of the Hoffman-Singleton graph in the sense of [6] where they are of fundamental importance for the code construction. These cocliques were also used by Hafner [13] in his alternative construction of the Higman-Sims graph from the Hoffman-Singleton graph which is used in Sage [34].

Note: For better reading in the figures the matrix entries " 0 " are printed as "." .

\section{The codes of length 100 which admit $\mathrm{G}=\mathrm{HS}$}

In this section all linear codes $C$ of length 100 which admit $G$ in the sense of [20] are determined.

Let $E$ be a stem cover of $G$ and $E_{\alpha}$ be the inverse image of the stabilizer $G_{\alpha}$. Then by $[20,3.2]$, all linear codes over a field $F$ admitting $G$ as a permutation group are obtained by the following procedure:

Induce up to $E$ all 1-dimensional $F E_{\alpha}$-modules. The submodules of the resulting $F E$-modules provide for a complete list of codes admitting $(G, \Omega)$ as permutation group.

In our case $G=$ HS there exists only one 1-dimensional $F E_{\alpha}$-module, namely the trivial on $F$, which induces up to the permutation module. This result is stated in [29] without reference. Since it is relevant for our purpose, we indicate a proof.

\section{(2.1) Lemma.}

Let $E$ be a stem cover of $G=\mathrm{HS}$ and let $E_{\alpha}$ be the inverse image of $G_{\alpha} \cong M_{22}$ under the canonical epimorphism $E \rightarrow G$. Then $E_{\alpha}$ is a stem extension of $G_{\alpha}$.

(Note that $H_{2}\left(M_{22}\right) \cong Z_{12}$ by a theorem of Mazet [26].) 


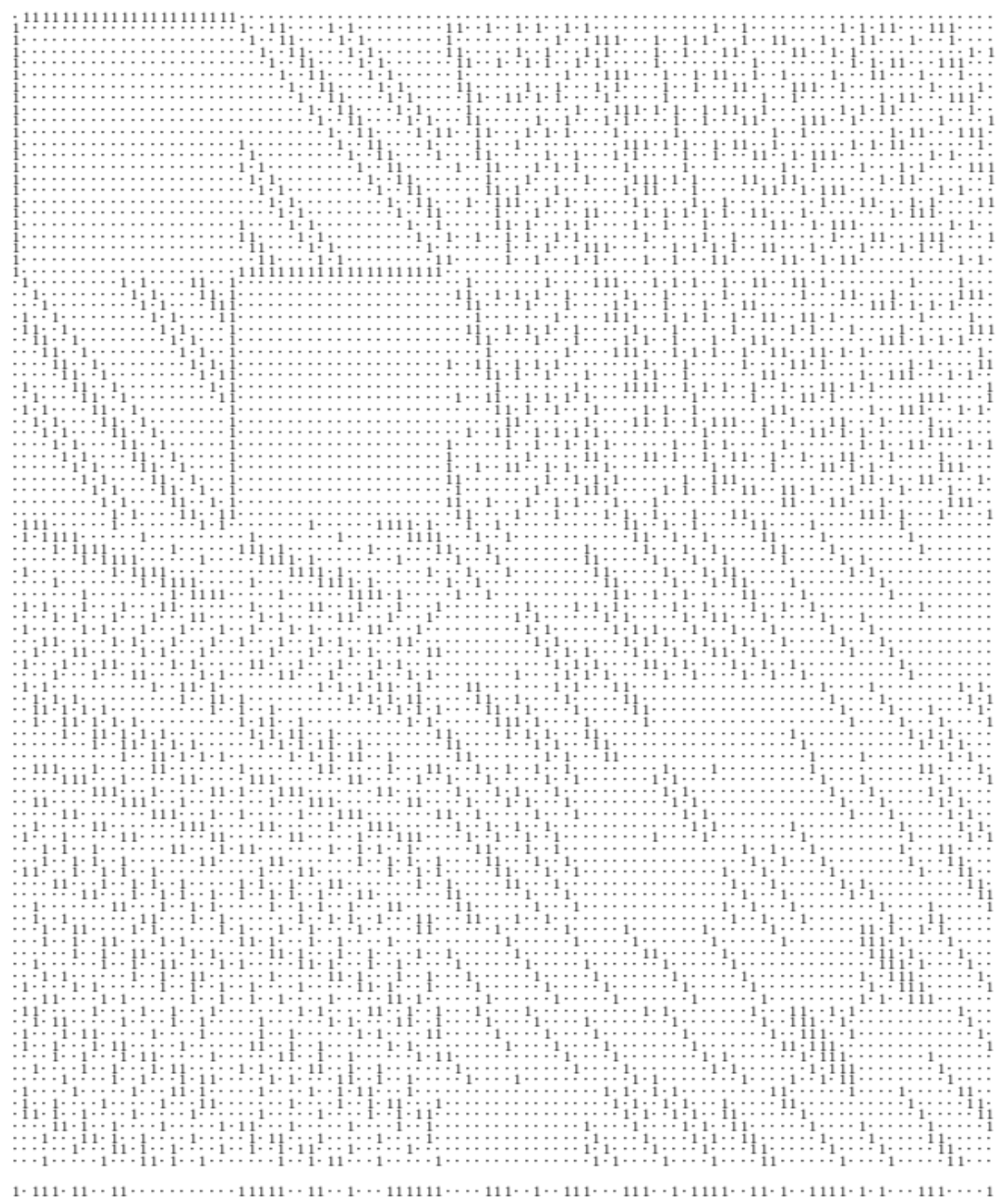

Figure 1: An adjacency matrix of the Higman-Sims graph and a Higman vector.

Proof. By [25] $G$ has exactly two conjugacy classes of involutions, say $2_{1}$ and $2_{2}$. The involutions in $2_{1}$ have exactly 20 fixed-points in $\Omega$ while the involutions in $2_{2}$ act fixedpoint freely on $\Omega$. Thus any involution in $2_{2}$ moves exactly $100 \equiv 4(\bmod 8)$ points. Following Griess [11] we consider now the stem cover $\widehat{A}$ of $\operatorname{Alt}_{\Omega} \geqslant G$, and we conclude that the inverse image $E$ of $G$ under the canonical epimorphism $\widehat{A} \rightarrow \operatorname{Alt}_{\Omega}$ is a stem extension. By [27] $E$ is the stem cover of $G$ (uniquely determined up to isomorphism).

To be explicit, Griess' argument shows that any inverse image $\widehat{t}$ of an involution $t \in 2_{2}$ 


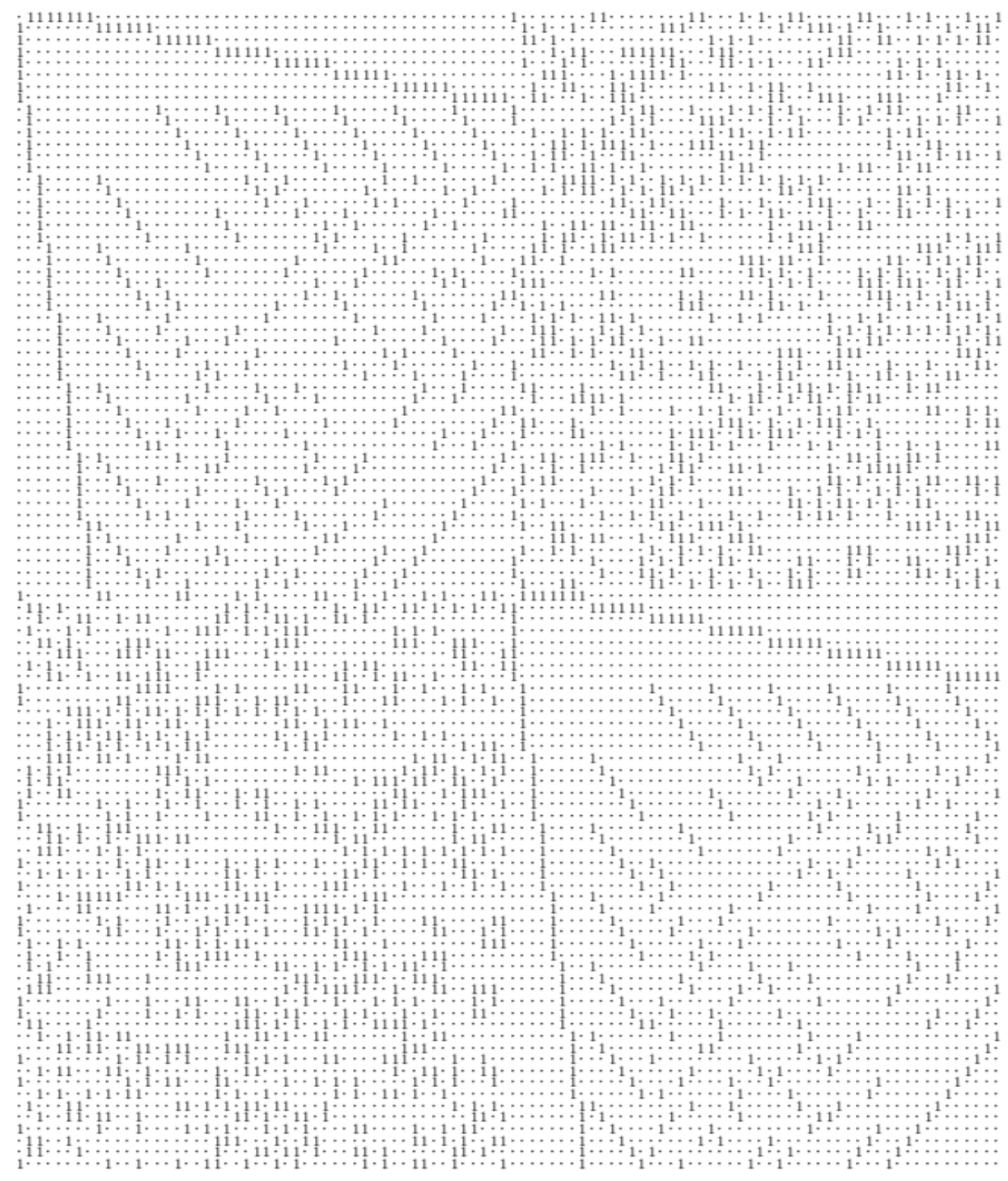

Figure 2: An adjacency matrix of the Higman-Sims graph displaying also the HoffmanSingleton graph.

is an element of order 4 such that $\widehat{t}^{2}=z$, where $\langle z\rangle=Z(A)=Z(E)$, see [18]. Now let $G_{\alpha}=M$ and let $h$ be an $\alpha$-hexad. By [25] the stabilizer $H$ of $h$ in $G$ is a semidirect product of $H_{1} \cong \Sigma_{6}$ by $N \cong Z_{2}^{4}$ such that $H_{2}=H_{1} \cap M \cong A_{6}$ acts irreducibly on $N$; of course, $\mathrm{NH}_{2}=H \cap M$ and $|H: H \cap M|=2$. We claim that there exists an involution $t \in 2_{2}$ such that $H=(H \cap M)\langle t\rangle$. To see this fact we recall the following results of [25]:

Let $S$ be a Sylow 2-subgroup of $G$ containing a Sylow 2-subgroup $S_{2}$ of $M . S$ contains an elementary abelian subgroup $V$ of order 4 whose involutions all belong to $2_{2}$. Since 
$S_{2} \cap V=1$ we conclude that $S=S_{2} V . S_{2}$ is contained in a Sylow 2-subgroup $S_{1}$ of $H$, therefore $S_{1} \cap V=\langle t\rangle$ for some involution $t \in 2_{2}$ and the claim is established.

We denote by ${ }^{\wedge}$ any inverse image under the canonical epimorphism $E \rightarrow G$. Since $\widehat{t}^{2}=z$ we infer that $\widehat{H}$ is stem extension of $H$. It is fairly obvious that $\widehat{N}$ is elementary abelian (recall that $\widehat{N} /\langle z\rangle \cong Z_{2}^{4}$ is an irreducible module). Since $\widehat{H}$ is a stem extension, $\widehat{N}$ is an indecomposable $\widehat{H} / \widehat{N}$-module where $\widehat{H} / \widehat{N} \cong \operatorname{Sym}_{6}$. But then $N$ is also an indecomposable $\widehat{H \cap M} / \widehat{N}$-module where $\widehat{H \cap M} / \widehat{N} \cong \mathrm{Alt}_{6}$, as one can see by restricting the natural permutation module over $\mathbb{F}_{2}$ from $\Sigma_{6}$ to $A_{6}$. Consequently we infer that $\widehat{H \cap M}$ is a stem extension of $H \cap M$ and so also $\widehat{M}=E_{\alpha}$ is a stem extension of $G_{\alpha}$.

As a consequence of (2.1) and [20, (3.2)], every code admitting $G=$ HS may be viewed as a submodule of the permutation module of $(G, \Omega)$. So the next step is to determine all such submodules. Clearly, we may consider first the case of characteristic 0 and then proceed by reduction modulo the prime characteristic $p$. In the modular case we first determine those submodules which are kernels or images of module endomorphisms; this type of submodules will be called endo-submodules. The complete lattice of submodules then is obtained from relations between endo-submodules, their perpendicular spaces and some extra considerations.

We recall from Section 1 the notations: $\Omega$ denotes the set of vertices of the HigmanSims graph, the Higman-Sims simple group $G=$ HS has the orbitals $\Delta_{0}, \Delta_{1}, \Delta_{2}$ where $\left|\Delta_{i}(\alpha)\right|=1,22,77$ respectively. $V_{0}, V_{1}, V_{2}$ are the matrices in the centralizer algebra of $(G, \Omega)$ corresponding to the orbitals $\Delta_{0}, \Delta_{1}, \Delta_{2}$ and $v_{i}$ denotes the endomorphism of the permutation module $F \Omega$ associated with the matrix $V_{i}$ or the orbital $\Delta_{i}$. As a general convention we write $\Delta=\Delta_{1}$ and $v=v_{1}$.

Clearly, the endomorphism algebra $E(F \Omega):=\operatorname{End}_{F G}(F \Omega)$ has basis $\left(v_{0}, v_{1}, v_{2}\right)$ where $v_{0}=\operatorname{id}_{F \Omega}$.

The right regular representation of $E(F \Omega)$ on itself defines a faithful matrix representation of $E(F \Omega)$ into $F^{3 \times 3}$ by

$$
a \mapsto\left(a_{i k}\right) \text { where } v_{i} a=\sum a_{i k} v_{k}
$$

The matrices $S_{j}=\left(\left(v_{j}\right)_{i k}\right)$ are the intersection matrices of the graphs $\left(\Omega, \Delta_{j}\right)$ in the sense of D.G. Higman [14] if char $F=0$. The structure of the Higman-Sims graph gives the following values:

$$
S_{0}=\mathrm{I}_{3}, \quad S_{1}=\left[\begin{array}{ccc}
0 & 1 & 0 \\
22 & 0 & 6 \\
0 & 21 & 16
\end{array}\right], \quad S_{2}=\left[\begin{array}{ccc}
0 & 0 & 1 \\
0 & 21 & 16 \\
77 & 56 & 60
\end{array}\right] \text {. }
$$

(In abuse of notation $m$ also denotes the element $m \cdot 1$ in the field $F$ for any integer m.)

$S_{1}$ has eigenvalues $22,-8,2$ and $S_{2}$ has eigenvalues $77,7,-3$. By construction, $E(F \Omega)$ is isomorphic to the algebra $A$ generated by $S_{0}, S_{1}$ (and $S_{2}$ ). Therefore we get the following. 


\section{(2.2) Proposition.}

Suppose $F$ is a field of characteristic 0 . Then the following hold:

(1) $F \Omega={ }_{F G} C \oplus X \oplus Y$ is the unique decomposition of $F \Omega$ into irreducible $F G$ submodules, where $\operatorname{dim} C=1, \operatorname{dim} X=22$ and $\operatorname{dim} Y=77$.

(2) The eigenvalues of the $v_{i}$ are distributed as follows:

\begin{tabular}{|c|ccc|}
\hline & $v_{0}$ & $v_{1}$ & $v_{2}$ \\
\hline$C$ & 1 & 22 & 77 \\
$X$ & 1 & -8 & 7 \\
$Y$ & 1 & 2 & -3 \\
\hline
\end{tabular}

(3) The projections in $E(F \Omega)$ onto the irreducible submodules $C, X, Y$ are given by

$$
\pi_{C}=\frac{1}{100}\left(v_{0}+v_{1}+v_{2}\right), \pi_{X}=\frac{1}{100}\left(22 v_{0}-8 v_{1}+2 v_{2}\right), \pi_{Y}=\frac{1}{100}\left(77 v_{0}+7 v_{1}-3 v_{2}\right) .
$$

Furthermore we have

$$
C=\operatorname{Im}\left(v_{0}+v_{1}+v_{2}\right), X=\operatorname{Im}\left(11 v_{0}-4 v_{1}+v_{2}\right), Y=\operatorname{Im}\left(77 v_{0}+7 v_{1}-3 v_{2}\right) .
$$

Proof. The algebra $A$ is generated by $S_{1}$ (or $S_{2}$ ); $A$ is commutative, semisimple and 3 -dimensional over $F$. The same holds for $E(F \Omega) \cong A$. Since $F \Omega$ is a completely reducible $F G$-module and since $v_{1}$ has 3 distinct eigenvalues, the permutation module $F \Omega$ decomposes into a sum of 3 absolutely irreducible submodules $C, X$ and $Y$. We choose the notation so that $C$ is the eigenspace of $v_{1}$ belonging to the eigenvalue 22 and that $X$ is the eigenspace of $v_{1}$ belonging to the eigenvalue $-8 . \quad j=v_{0}+v_{1}+v_{2}$ is the endomorphism with matrix $J=V_{0}+V_{1}+V_{2}$ whose entries are all 1 . Hence we may conclude that the eigenvalues of the $v_{i}$ are distributed as claimed in (2). Obviously $\operatorname{dim} C=1$ holds. Let $x=\operatorname{dim} X$ and $y=\operatorname{dim} Y$. Then we have $0=\operatorname{trace} v_{1}=22-8 x+2 y$ and $2,200=\operatorname{trace} v_{1}^{2}=484+64 x+4 y$, since $V_{1}$ is symmetric (see [38, 28.10]). It follows that $x=22$ and $y=77$ as asserted in (1). From (2) it now may be deduced that the projection idempotents in $E(F \Omega)$ onto the irreducible submodules are those given in (3). The rest follows.

The submodule structure of $F \Omega$ is completely clear by the results of (2.2) if $\operatorname{char} F=$ 0 . If $\operatorname{char} F$ does not divide $|G|=44,352,000=2^{9} \cdot 3^{2} \cdot 5^{3} \cdot 7 \cdot 11$ then we have the same situation. Note that in all these cases the irreducible submodules are unique and absolutely irreducible over the prime field.

For notation of characters and Brauer characters we use the following conventions:

If $\chi$ denotes a (Brauer) character of $G=$ HS then $\chi_{m}$ denotes the restriction of $\chi$ to $G_{\alpha} \cong M_{22}$. If there is no ambiguity characters (of any group) are denoted by their degrees.

\section{(2.3) Lemma.}

Let 100 be the permutation character of $G=\mathrm{HS}$ acting on $\Omega$ and let 1,22 and 77 denote 
the characters belonging to the modules $C, X$ and $Y$ respectively. Then the following holds:

$$
\begin{aligned}
\text { (i) } 22_{m} & =1+21 . \\
\text { (ii) } 77_{m} & =1+21+55 . \\
\text { (iii) } 100_{m} & =3 \cdot 1+2 \cdot 21+55 .
\end{aligned}
$$

Proof. The assertion follows from the well known suborbit structure of $G=$ HS and of $M_{22}$ acting on the points and blocks of $\mathcal{W}_{22}$, see Section 1.

\section{(2.4) Proposition.}

If $F$ is a field of prime characteristic $p \neq 2,5$ then all assertions of (2.2) hold (with scalars read modulo $p$ ).

Proof. It suffices to consider the case $F=\mathbb{F}_{p}$ for $p \in\{3,7,11\}$. Since $p$ does not divide 100 the reductions modulo $p$ of the projections $\pi_{C}, \pi_{X}$ and $\pi_{Y}$ are distinct pairwise orthogonal idempotents. So it follows that $F \Omega$ is the direct sum of the reductions modulo $p \bar{C}, \bar{X}$ and $\bar{Y}$ of $C, X$ and $Y$. It is now sufficient to show that $\bar{C}, \bar{X}$ and $\bar{Y}$ are absolutely irreducible. This is trivial for $\bar{C}$. Of course, we have $\operatorname{dim} \bar{X}=22$ and $\operatorname{dim} \bar{Y}=77$.

(i) If $p=11$ then $X$ and $Y$ both belong to blocks of defect 0 . We immediately conclude that $X$ and $Y$ are absolutely irreducible.

(ii) Let $p=7$. Then $Y$ belongs to a block of defect 0 , and again $\bar{Y}$ must be absolutely irreducible. Let $\beta$ denote the Brauer character of $\bar{X}$. Then by James' [19, 5.1], we have $\beta_{m}=1+21$. Again it follows that $\bar{X}$ is absolutely irreducible, since $\bar{C}$ contains the fixed-points of $G$ in $F \Omega$.

(iii) Let $p=3$. By James' $[19,7.3]$, the characters 21 and 55 of $M_{22}$ remain irreducible when reduced modulo 3. Hence we may conclude from (2.2) that $\bar{X}$ and $\bar{Y}$ are again absolutely irreducible, using the obvious fact that $\bar{X}$ and $\bar{Y}$ are self-dual modules.

It remains to consider the case that $\operatorname{char} F$ divides 100 . At first we consider the direct decomposition of $F \Omega$.

\section{(2.5) Proposition.}

If char $F=p \in\{2,5\}$ then the following hold:

(1) $F \Omega$ is absolutely indecomposable.

(2) $E(F \Omega)$ is a local F-algebra such that $\operatorname{dim} E(F \Omega) / J(E(F \Omega))=1$.

(3) If $p=5$ then $J(E(F \Omega))$ has $F$-basis $\left(w, w^{2}\right)$ where $w=2 v_{0}-v_{1}$. If $p=2$ then $J(E(F \Omega))$ has $F$-basis $\left(v_{1}, v_{0}+v_{1}+v_{2}\right)$. 
Proof. (1) and (2) follow from (3). (3) may be established by direct computation.

\section{(2.6) Proposition.}

Let $\operatorname{char} F=5$ and set $w=2 v_{0}-v_{1} \in E(F \Omega)$. Let $H_{100}=F \Omega, H_{0}=0, H_{99}=\operatorname{Ker} w^{2}$, $H_{1}=\operatorname{Im} w^{2}, H_{77}=\operatorname{Ker} w, H_{23}=\operatorname{Im} w, H_{78}=H_{77}+H_{23}$ and $H_{22}=H_{77} \cap H_{23}$. Then the following hold.

(1) $\left\{H_{i} \mid i \in\{0,1,22,23,77,78,99,100\}\right\}$ is the complete set of $F G$-submodules of $F \Omega$ and $\operatorname{dim} H_{i}=i$ for all $i$.

(2) Every composition factor of $F \Omega$ remains irreducible when restricted to $G_{\alpha} \cong M_{22}$.

(3) $H_{i}^{\perp}=H_{100-i}$ for all $i$.

Proof. From James' [19, 4.3], it follows that the characters 1, 21 and 55 of $G_{\alpha} \cong M_{22}$ remain irreducible when reduced modulo 5 . Therefore we conclude from $(2.2)$ that in a composition series of $F \Omega$ viewed as an $F G$-module we have exactly 3 (trivial) composition factors of dimension 1, 2 composition factors of dimension 21 and 1 composition factor of dimension 55 .

From (2.5) it follows that the $F G$-submodules $H_{i}$ of $F \Omega$ defined above are pairwise distinct and that $H_{1} \leqslant H_{22}$ and $H_{78} \leqslant H_{99}$. It follows that $0=H_{0}<H_{1}<H_{22}<H_{k}<$ $H_{78}<H_{99}<H_{100}=F \Omega$, where $k \in\{23,77\}$ are two composition series of $F \Omega$ as an $F G$-module. hence (2) holds.

The matrices in the centralizer algebra of $F \Omega$ which is spanned by $V_{0}, V_{1}$ and $V_{2}$ are all symmetric. Therefore $\operatorname{Ker} a=(\operatorname{Im} a)^{\perp}$ for any $a \in E(F \Omega)$. Referring to the definition of the $H_{i}$ we see that (3) holds.

Using (3), from $\operatorname{dim} E(F \Omega)=3$ it follows that $H_{23}$ and $H_{100} / H_{77}$ are uniserial. We claim that also $H_{77}$ (and therefore $H_{100} / H_{23} \cong H_{77}^{*}$ ) is uniserial.

First of all, from (2.5) it follows that $H_{77}$ is indecomposable. For the claim it is sufficient to prove that also $H_{77} / H_{1}$ is indecomposable. Otherwise there would be an $F G$-submodule $Y$ of $H_{77}$ such that $H_{77}=Y+H_{22}$ and $Y \cap H_{22}=H_{1}$. For $M=G_{\alpha}$ we have the double module decomposition

$$
G:(M, M)=\{M, M x M, M y M\}
$$

for some $x, y, \in G$, notation so that $|\{z M \mid z M \subseteq M x M\}|=22$. The $F M$-Module $Y / H_{1}$ is irreducible in a block of defect 0 , hence $Y / H_{1}$ is a projective $F M$-module. It follows that there must exist an irreducible $F M$-submodule $X$ of dimension 55 such that $Y=X \oplus H_{1}$. But then $Y_{0}=\bigcap\{X x g \mid g \in M\}$ would be a proper $F M$-submodule of $Y$ of codimension at most 22 and so it would follow that $X=Y_{0}$, therefore in particular $X=X x$. Since $G=\langle M, x\rangle, X$ would be an $F G$-submodule, contradicting the indecomposability of $H_{77}$.

Now let $U$ be any $F G$-submodule of $F \Omega$. We have to show that $U$ is one of the $H_{i}$ :

By taking orthogonal subspaces, if necessary, we may suppose $\operatorname{dim} U \leqslant 50$ because of (3). Since $F \Omega / H_{23}$ has a unique minimal irreducible submodule of dimension 55, it follows $U \leqslant H_{23}$. But then $U=H_{i}$ for some $i$, hence (1) holds. 
The only remaining case is now char $F=2$. Of course, this is the most interesting case in view of possible applications. It turns out that it is also highly relevant for the combinatorial properties of the Higman-Sims graph and for the structure of $G=$ HS. We set again $\mathbb{1}=\sum \Omega \in F \Omega$.

\section{(2.7) Proposition.}

If $F=\mathbb{F}_{2}$ the following hold:

(1) $F \Omega$ has precisely the following endo-submodules $H_{i}$ with $\operatorname{dim} H_{i}=i$ :

$$
\begin{aligned}
& H_{100}=F \Omega, \quad H_{0}=0, \quad H_{99}=\operatorname{Ker}\left(v_{0}+v_{1}+v_{2}\right), \\
& H_{1}=\operatorname{Im}\left(v_{0}+v_{1}+v_{2}\right), \quad H_{78}=\operatorname{Ker} v_{1}, \quad H_{22}=\operatorname{Im} v_{1} .
\end{aligned}
$$

These submodules form a series $H_{0}<H_{1}<H_{22}<H_{78}<H_{99}<H_{100}$.

(2) For every $a \in E(F \Omega)$ we have $\operatorname{Ker} a=(\operatorname{Im} a)^{\perp}$, thus $H_{i}^{\perp}=H_{100-i}$ for the endosubmodules $H_{i}$.

(3) $H_{21}=\left\{x \mid x \in H_{22}\right.$ and $\left.w(x) \equiv 0(\bmod 4)\right\}$ is an FG-submodule of codimension 1 in $\mathrm{H}_{22}$.

Set $H_{79}=H_{21}{ }^{\perp}$. Then $\operatorname{dim} H_{i}=i$ for $i \in\{21,79\}$ and $0=H_{0}<H_{1}<H_{21}<$ $H_{22}<H_{78}<H_{79}<H_{99}<H_{100}=F \Omega$ is a composition series of $F \Omega$ as an $F G$ module. The dimensions of the composition factors in this composition series are 1,20,1,56,1,20,1. All composition factors of $F \Omega$ are absolutely irreducible.

(4) $F \Omega$ has exactly one $F G$-submodule $H_{23}$ of dimension 23. Set $H_{77}=H_{23}{ }^{\perp}$; then also $\operatorname{dim} H_{77}=77$. We have $H_{22}<H_{23}<H_{79}$ and $H_{21}<H_{77}<H_{78}$.

Between $H_{21}$ and $H_{23}$ there are exactly 3 distinct $F G$-submodules $H_{22}, H_{22}^{\prime}$ and $H_{22}^{\prime \prime}$. Set $H_{78}^{\prime}=\left(H_{22}^{\prime}\right)^{\perp}$ and $H_{78}^{\prime}=\left(H_{22}^{\prime \prime}\right)^{\perp}$. Then $\operatorname{dim} H_{i}^{\prime}=i=\operatorname{dim} H_{i}^{\prime \prime}$ for $i \in\{22,78\}$ and $H_{78}, H_{78}^{\prime}$ and $H_{78}^{\prime \prime}$ are the only $F G$-submodules between $H_{77}$ and $H_{79}$. We have $H_{22}^{\prime}<H_{78}^{\prime}$ and $H_{22}^{\prime \prime}<H_{78}^{\prime \prime}$.

(5) $\left\{H_{0}, H_{1}, H_{21}, H_{22}, H_{22}^{\prime}, H_{22}^{\prime \prime}, H_{23}, H_{77}, H_{78}, H_{78}^{\prime}, H_{78}^{\prime \prime}, H_{79}, H_{99}, H_{100}\right\}$ is the complete set of $F G$-submodules of $F \Omega$.

(6) $H_{22}$ is generated by all adjacency vectors $\Delta(\beta)$ of the Higman-Sims graph; $H_{78}$ is generated by all $\beta$-hexads, $\beta \in \Omega$.

(7) $H_{21}$ (respectively $H_{77}$ ) is the set of all sums of an even number of adjacency vectors of the Higman-Sims graph (respectively of $\beta$-hexads).

(8) $H_{79} \backslash H_{78}=\{x \mid x \in F \Omega$ and $(x, \Delta(\beta))=1$ for all $\beta \in \Omega\}$,

$$
H_{23} \backslash H_{22}=\{x \mid x \in F \Omega \text { and }(x, h)=1 \text { for all } \beta \text {-hexads } h, \beta \in \Omega\} .
$$


(9) For any heptad $m$ of $\mathcal{W}_{22}$ in the sense of (1.4) let $x(m)=\alpha+m+B_{1}(m)$ be the corresponding Higman vector. Let $\mathcal{M}^{\prime}$ and $\mathcal{M}^{\prime \prime}$ be the two orbits of $G_{\alpha} \cong M_{22}$ on the set of heptads in $\mathcal{W}_{22}$ (see Section 1). Then, with appropriate notation, we have

$$
H_{22}^{\prime}=H_{21}+F x(m) \text { for any } m \in \mathcal{M}^{\prime} \text { and } H_{22}^{\prime \prime}=H_{21}+F x(m) \text { for any } m \in \mathcal{M}^{\prime \prime} \text {. }
$$

(10) $H_{21}=\left\{x \mid x \in H_{23}\right.$ and $\left.w(x) \equiv 0(\bmod 4)\right\}$.

(11) The action of $G$ on $F \Omega$ extends in a natural way to $\bar{G} \cong \operatorname{Aut}(G)$. Every $H_{i}$ is invariant under $\bar{G}$, but $\bar{G}$ interchanges the submodules $H_{22}^{\prime}, H_{22}^{\prime \prime}$ and $H_{78}^{\prime}, H_{78}^{\prime \prime}$.

In the case of arbitrary fields $F \supseteq \mathbb{F}_{2}$ we have essentially the same situation, since $F \Omega \cong$ $F \otimes_{\mathbb{F}_{2}} \mathbb{F}_{2} \Omega$ and almost all completely reducible factors are multiplicity-free. Only the factors $H_{23} / H_{21}$ and $H_{79} / H_{77}$ yield a number of submodules increasing with the size of the field $F$.

Remark. Because of the last statement of the Proposition, and since all composition factors of $\mathbb{F}_{2} \Omega$ as an $\mathbb{F}_{2} G$-module are absolutely irreducible, we restrict our attention in characteristic 2 to the case $F=\mathbb{F}_{2}$. It does not seem promising to consider larger fields $F$ for code theoretic applications.

Proof.

(i) From (2.5) it follows that $R=J(E(F \Omega))=\left\{0, v_{1}, v_{0}+v_{2}, v_{0}+v_{1}+v_{2}\right\}$. It is immediate that $R^{2}=0$. We also see that the submodules $H_{i}$ defined in (1) are endo-submodules and that for all $a \in E(F \Omega) \operatorname{Ker} a=(\operatorname{Im} a)^{\perp}$, since the matrix belonging to $a$ is symmetric. From $R^{2}=0$ we infer that $0=H_{0} \leqslant H_{1}, H_{22} \leqslant$ $H_{78}, H_{99} \leqslant H_{100}=F \Omega$. To establish that there are no other endo-submodules it suffices to show that $\operatorname{Im}\left(v_{0}+v_{2}\right)=\operatorname{Im} v_{1}=H_{22}$. Clearly $H_{1}=F \mathbb{1}$ holds. Since $V_{1}$ and $V 0+V_{2}$ are complementary 0,1-matrices, we have $\operatorname{Im}\left(v_{0}+v_{2}\right)+H_{1}=$ $\operatorname{Im} v_{1}+H_{1}$. So it is sufficient to show that $H_{1} \leqslant \operatorname{Im} v_{1}=H_{22}$. If $H_{1} \nless H_{22}$ then $U=H_{22} \cap \operatorname{Im}\left(v_{0}+v_{2}\right) \cong U^{*}$ would be the image of an endomorphism $u \in R$, which is impossible. Therefore we have $\operatorname{Im}\left(v_{0}+v_{2}\right)=H_{22}$ as claimed. It also follows that we have a series $0=H_{0}<H_{1}<H_{22}<H_{78}<H_{99}<H_{100}=F \Omega$ of $F G$ submodules. Of course, $\operatorname{dim} H_{0}=0, \operatorname{dim} H_{1}=1, \operatorname{dim} H_{99}=99$ and $\operatorname{dim} H_{100}=100$. We assert that $\operatorname{dim} H_{22}=22$ and $\operatorname{dim} H_{78}=78$. From $(2.2)(3)$ it follows that $H_{22}$ is the reduction modulo 2 of $X \cap \mathbb{Z} \Omega$, hence $\operatorname{dim} H_{22} \leqslant \operatorname{dim} X=22$. From (1.3) it follows that the matrix $V_{1}$ of $v_{1}$ which is the adjacency matrix of the Higman-Sims graph over $F=\mathbb{F}_{2}$ has rank at least 22. Therefore we have $\operatorname{dim} H_{22}=22$ and $\operatorname{dim} H_{78}=78$. (1) and (2) of the proposition are now proved completely.

(ii) Since $H_{22} \leqslant H_{22}{ }^{\perp}=H_{78} \leqslant H_{99}=\{x \mid x \in F \Omega$ and $w(x) \equiv 0(\bmod 2)\}$, we get that

$$
H_{21}=\left\{x \mid x \in H_{22} \text { and } w(x) \equiv 0 \quad(\bmod 4)\right\}
$$

is an FG-submodule of dimension 21, contained in $H_{22}$. Set $H_{79}=H_{21}{ }^{\perp}$. Of course, then $\operatorname{dim} H_{79}=\operatorname{dim} H_{21}{ }^{\perp}=100-21=79$ holds. The series of $F G$-submodules of 
$F \Omega 0=H_{0}<H_{1}<H_{21}<H_{22}<H_{78}<H_{79}<H_{99}<H_{100}=F \Omega$ has successive factors of dimensions $1,20,1,56,1,20,1$ respectively. According to J. Thackray [35] (see also the atlas of Brauer Characters [2]) all these factor modules are absolutely irreducible. (For the factors of dimension 1 and 20 this is quite elementary; for the factor of dimension 56 a direct elementary proof should also be possible. Note that we can use Lemma (2.3).) The proof of (3) is now complete.

(iii) From (2) and (1) it follows that the $F G$-modules $H_{100} / H_{78}$ and $H_{22}$ are self-dual and uniserial. Proposition (1.1) gives that the reduction modulo 2 of $(X+C) \cap \mathbb{Z} \Omega$ is a 23-dimensional submodule $H_{23}$ of $F \Omega . H_{23}$ contains $H_{22}$ which is the reduction of $X \cap \mathbb{Z} \Omega$. Set $H_{77}=H_{23}{ }^{\perp}$. Of course, we then have $\operatorname{dim} H_{23}=23$ and $\operatorname{dim} H_{77}=77$. Since $H_{23} \cap H_{78}=H_{22}$ we obtain from the structure of $H_{100} / H_{78}$ that $H_{79}=H_{78}+H_{23}$ and therefore $H_{77} \cap H_{22}=H_{21}$. We claim that $H_{77}$ is uniserial. Otherwise there were an $F G$-submodule $Y$ such that $H_{77}=Y+H_{21}$ and $Y \cap H_{21}=H_{1}$, and we had the direct decomposition $H_{99} / H_{1}=Y / H_{1} \oplus Y / H_{1}$ into submodules of dimensions 56 and 42. Viewing $H_{99} / H_{1}$ as an $F G_{\alpha}$-module this contradicts (1.15) by the Krull-Schmidt theorem. Since $H_{100} / H_{23} \cong H_{77}{ }^{*}$, also $H_{100} / H_{23}$ is uniserial.

By counting we see that there are besides $H_{22}$ precisely two further $F G$-submodules $H_{22}^{\prime}$ and $H_{22}^{\prime \prime}$ between $H_{21}$ and $H_{23}$. We set $H_{78}^{\prime}=\left(H_{22}^{\prime}\right)^{\perp}$ and $H_{78}^{\prime \prime}=\left(H_{22}^{\prime \prime}\right)^{\perp}$. Then by duality $H_{78}, H_{78}^{\prime}$ and $H_{78}^{\prime \prime}$ are precisely the $F G$-submodules between $H_{77}$ and $H_{79}$. We have $H_{22}^{\prime}=H_{21}+F x^{\prime}$ for some vector $x^{\prime}$ of even weight. Since $H_{21}{ }^{\perp}=H_{79} \geqslant H_{22}^{\prime}$ it follows that $H_{22}^{\prime} \leqslant\left(H_{22}^{\prime}\right)^{\perp}=H_{78}{ }^{\perp}$. Similarly, also $H_{22}^{\prime \prime} \leqslant H_{78}^{\prime \prime}$.

(iv) Now let $X$ be any $F G$-submodule of $F \Omega$. We claim that $X=H_{i}$ or $X=H_{i}^{\prime}$ or $X=H_{i}^{\prime \prime}$ for some $i$. Since the set of the $H_{i}, H_{i}^{\prime}, H_{i}^{\prime \prime}$ is closed under orthogonal complements, we may assume that $\operatorname{dim} X \leqslant 50$. If $X$ were not contained in $H_{23}$ we would have $X+H_{23}>H_{23}$ and $\operatorname{dim}\left(X+H_{23} / H_{23}\right) \geqslant 56$, since $H_{100} / H_{23}$ contains a unique minimal $F G$-submodule of dimension 56, see (iii). This contradicts $\operatorname{dim} X \leqslant$ 50. Therefore $X \leqslant H_{23}$ holds. Taking into account that $H_{1}=\langle\mathbb{1}\rangle$ is the $F G$ submodule of $F \Omega$ containing all vectors fixed by the perfect group $G$ we now get immediately that $X=H_{i}$ or $X=H_{i}^{\prime}$ or $X=H_{i}^{\prime \prime}$ for some $i \leqslant 23$. (4) and (5) are now proved completely.

(v) Since $H_{22}=\operatorname{Im} v_{1}$ it is plainly clear that $H_{22}$ is generated by all the adjacency vectors of the Higman-Sims graph. From (1) it follows that every $\beta$-hexad is orthogonal to every adjacency vector $\Delta(\beta)$ of the Higman-Sims graph. Therefore the $F G$ submodule $H$ of $F \Omega$ generated by all $\beta$-hexads, $\beta \in \Omega$, is contained in $H_{78}=H_{22}{ }^{\perp}$. From (1.3) we infer that $H_{22}$ is contained in $H$, since $\Delta(\alpha)$ is a sum of $\alpha$-hexads. We shall show later in Section 3 (independently) that the minimum weight of $H_{22}$ is 22 . Therefore no $\beta$-hexad is contained in $H_{22}$, so it follows that $H=H_{78}$, since $H_{78} / H_{22}$ is irreducible. The proof of (6) is complete; (7) immediately follows from (3) and (6).

(vi) By the homomorphism theorem for $F G$-modules we have $H_{79} \backslash H_{78}=\{x \in F \Omega \mid$ $x v=\mathbb{1}\}$. Since $v$ is the linear extension of the map $\beta \mapsto \Delta(\beta)$ from (6) and 
(7) it follows that $H_{79} \backslash H_{78}=\{x \in F \Omega \mid(x, \Delta(\beta))=1$ for all $\beta \in \Omega\}$. Clearly $A=\{x \in F \Omega \mid(x, h)=1$ for all $\beta$-hexads $\}$ is contained in $H_{23} \backslash H_{22}$ by (7). Also $A+H_{22}=A$ holds. Since $\mathbb{1} \in H_{78}$ from (6) and elementary linear algebra it follows that $A \neq \emptyset$ and hence $A=H_{23} \backslash H_{22}$. The proof of (8) is complete.

(vii) Let $m$ be any heptad of $\mathcal{W}_{22}$ in the sense of Section 1 and let $x(m)=\alpha+m+B_{1}(m)$ be the corresponding Higman vector. From (1.10) it follows that $(x(m), h)=1$ for all $\beta$-hexads $h, \beta \in \Omega$. Therefore $x(m) \in H_{23} \backslash H_{22}$ follows from (8). Consequently we have $H_{23}=H_{22}+F x(m)$ and $H_{21}+F x(m) \in\left\{H_{22}^{\prime}, H_{22}^{\prime \prime}\right\}$.

$G=$ HS has two orbits $\mathcal{M}^{\prime}$ and $\mathcal{M}^{\prime \prime}$ in the set of heptads of $\mathcal{W}_{22}$. Independently, it will be shown later in (4.1), that if $m^{\prime} \in \mathcal{M}^{\prime}$ and $m^{\prime \prime} \in \mathcal{M}^{\prime \prime}$ then $x\left(m^{\prime}\right)+x\left(m^{\prime \prime}\right) \in$ $H_{22} \backslash H_{21}$. Hence we may choose the notation so that $H_{22}^{\prime}=H_{21}+F x\left(m^{\prime}\right)$ if $m^{\prime} \in \mathcal{M}^{\prime}$ and $H_{22}^{\prime \prime}=H_{21}+F x\left(m^{\prime \prime}\right)$ if $m^{\prime \prime} \in \mathcal{M}^{\prime \prime}$. Since the $G$-orbits $\mathcal{M}^{\prime}$ and $\mathcal{M}^{\prime \prime}$ are interchanged by $\overline{M_{22}} \cong \operatorname{Aut}\left(M_{22}\right)$ we also conclude that $\bar{G}$ interchanges $H_{22}^{\prime}$ and $H_{22}^{\prime \prime}$.

The assertions (9) and (11) now readily follow. The rest of the Proposition is clear.

We may collect the results of this section:

(2.8) Theorem.

Let $G=\mathrm{HS}$ be the Higman-Sims simple group in its rank 3 representation on $\Omega$ of degree 100. Then every linear code $C$ over a field $F$ admitting $G$ is obtained within isomorphy from one of the FG-submodules of the permutation module F $\Omega$ which are given in the propositions (2.2), (2.4), (2.6) and (2.7).

Proof. The theorem follows from [20, (3.2)], Lemma (2.1) and the preceding propositions.

The submodule structure of $H_{100}=\mathbb{F}_{2} \Omega$ is displayed in Figure 3.

\section{The binary linear codes of length 100 admitting $\mathrm{G}=\mathrm{HS}$ and their relation to the combinatorial structure of the Higman- Sims graph}

Our aim is to determine the weight structure of the binary linear codes of length 100 which admit the Higman-Sims group $G=$ HS and to discuss its combinatorial meaning. Throughout this section let $F=\mathbb{F}_{2}$. We may tacitly assume that $F \Omega$ is always the ambient space and that $\Omega$ is the ambient basis. By abuse of notation we denote these linear codes by the corresponding submodules of $F \Omega$, as introduced in Section 2. We may identify $F \Omega$ with the power set $\mathcal{P}(\Omega)$ in the canonical way whenever it is convenient.

Let $\mathbb{1}=\sum \Omega=\sum\{\beta \mid \beta \in \Omega\}$ denote the all 1 vector in $F \Omega$. We recall from Section 1 the notation $W_{i}(X)=\{x \in X \mid w(x)=i\}$ for $X \subseteq F \Omega$ and $0 \leqslant i \leqslant 100$. Set 


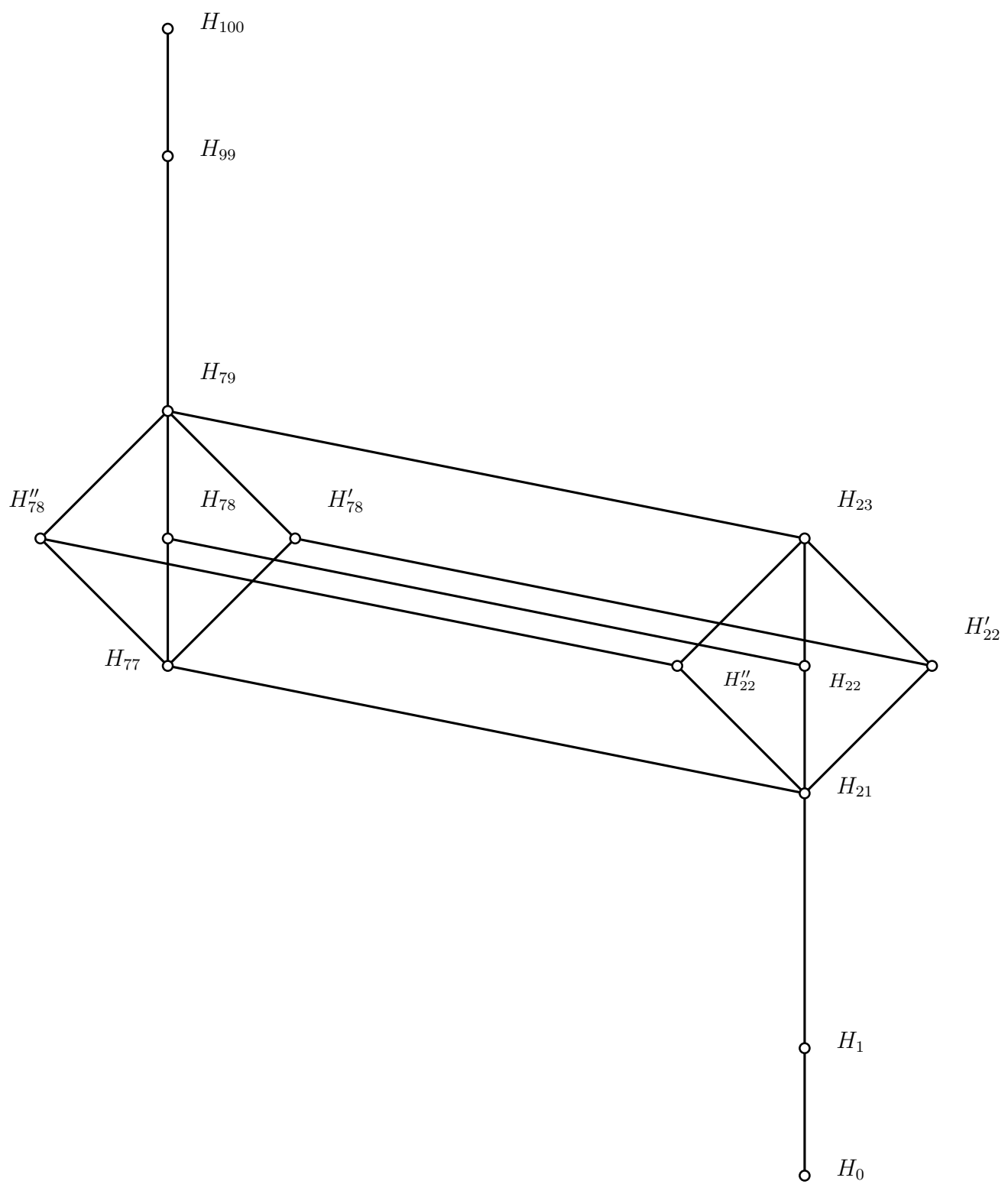

Figure 3: The binary permutation module of $G=\mathrm{HS}$ and its submodules. 
$w_{i}(X):=\left|W_{i}(X)\right|$. Let $m(X)$ denote the minimum (non-zero) weight of $X$ (whenever it is defined).

We begin by studying the vectors of small weight in $H_{79}, H_{78}$ and $H_{22}$. It will be useful for this purpose to have a classification of vectors in $F \Omega$ of weight at most 4 under the action of $G=$ HS.

\section{(3.1) Proposition.}

The $G$-orbits in $W_{i}(F \Omega)$ for $1 \leqslant i \leqslant 4$ are as follows:

(1) $G$ acts transitively on $W_{1}(F \Omega)=\Phi_{1},\left|\Phi_{1}\right|=100$.

(2) $W_{2}(F \Omega): G=\left\{\Phi_{21}, \Phi_{22}\right\}$, where $\left|\Phi_{21}\right|=1,100$ and $\left|\Phi_{22}\right|=3,850$.

(3) $W_{3}(F \Omega): G=\left\{\Phi_{31}, \Phi_{32}, \Pi_{33}\right\}$, where $\left|\Phi_{31}\right|=77,000,\left|\Phi_{32}\right|=61,000$ and $\left|\Phi_{33}\right|=$ 23,100 .

(4) $W_{4}(F \Omega): G=\left\{\Phi_{4, i} \mid 1 \leqslant i \leqslant 9\right\}$, where $\left|\Phi_{41}\right|=57,750,\left|\Phi_{42}\right|=616,000,\left|\Phi_{43}\right|=$ 231,000. $\left|\Phi_{44}\right|=1,386,000,\left|\Phi_{45}\right|=154,000,\left|\Phi_{46}\right|=924,000 . \quad\left|\Phi_{47}\right|=369,600$, $\left|\Phi_{48}\right|=154,000$ and $\left|\Phi_{49}\right|=28,875$.

Representatives of the orbits are given in the table on the following page.

In the table - - indicates two vertices not joined by an edge in the Higman-Sims graph, $\bullet-\bullet$ indicates two vertices joined by an edge in the Higman-Sims graph and $\bullet<\bullet$ as well as $\bullet \bullet$ indicate a vertex joined by edges to two distinct vertices etc.

The type of an element $x \in F \Omega$ has the obvious meaning indicated by the edges and non-edges in the support of $x$.

The term " $i$-vertical" for some $x \in F \Omega$ means that the maximum number of vertices $\xi \in \Omega$ such that $\operatorname{supp}(x) \subseteq \Delta(\xi)$ is precisely $i$.

\begin{tabular}{|c|c|c|}
\hline orbit & type of orbit element & orbit length \\
\hline$\Phi_{1}$ & $\bullet$ & 100 \\
\hline$\Phi_{21}$ & $\bullet-\bullet$ & 1,100 \\
\hline$\Phi_{22}$ & & 3,850 \\
\hline$\Phi_{31}$ & $\bullet \bullet$ & 77,000 \\
\hline$\Phi_{32}$ & $\bullet-\bullet$ & 61,600 \\
\hline$\Phi_{33}$ & $\bullet-\bullet-\bullet$ & 23,100 \\
\hline$\Phi_{41}$ & - • • • 2-vertical & 57,750 \\
\hline$\Phi_{42}$ & - $-\bullet 1$-vertical & 616,000 \\
\hline$\Phi_{43}$ & - $-\bullet 0$-vertical & 231,000 \\
\hline$\Phi_{44}$ & $\bullet-\bullet \bullet \bullet$ & $1,386,000$ \\
\hline$\Phi_{45}$ & $\bullet-\bullet \bullet \bullet-\bullet$ & 154,000 \\
\hline$\Phi_{46}$ & $\bullet-\bullet-\bullet \bullet$ & 924,000 \\
\hline$\Phi_{47}$ & $\bullet-\bullet-\bullet-\bullet$ & 369,600 \\
\hline$\Phi_{48}$ & & 154,000 \\
\hline$\Phi_{49}$ & $\bullet<>\bullet$ & 28,875 \\
\hline
\end{tabular}


Proof. Since the Higman-Sims graph is triangle-free $(\lambda=0)$ it is readily shown that all the vectors $x \in W_{i}(F \Omega)(1 \leqslant i \leqslant 4)$ belong to one of the types shown in the table. The known facts about the action of $G_{\alpha} \cong M_{22}([16,22])$ are now sufficient to establish the proposition.

It is fairly easy to compute the weight of the images $x v \in H_{22}$ of the vectors $x$ given in (3.1). Of course, the weight depends only on the $G$-orbit.

\section{(3.2) Lemma.}

The Hamming weight $w(x v)$ of the vectors $x v$ for $x \in W_{i}(F \Omega)(1 \leqslant i \leqslant 4)$ is given by the following table where $x$ is contained in the indicated $G$-orbit.

\begin{tabular}{|c|cccccc|}
\hline orbit & $\Phi_{1}$ & $\Phi_{21}$ & $\Phi_{22}$ & $\Phi_{31}$ & $\Phi_{32}$ & $\Phi_{33}$ \\
$w(x v)$ & 22 & 44 & 32 & 38 & 42 & 54 \\
\hline
\end{tabular}

\begin{tabular}{|c|ccccccccc|}
\hline orbit & $\Phi_{41}$ & $\Phi_{42}$ & $\Phi_{43}$ & $\Phi_{44}$ & $\Phi_{45}$ & $\Phi_{46}$ & $\Phi_{47}$ & $\Phi_{48}$ & $\Phi_{49}$ \\
$w(x v)$ & 32 & 40 & 48 & 44 & 40 & 48 & 52 & 60 & 64 \\
\hline
\end{tabular}

Proof. If $x=\sum x_{\beta} \beta$ then $x v=\sum\left\{\Delta(\beta) \mid x_{\beta} \neq 0\right\}$. Elementary calculations in the Higman-Sims graph easily give the weights.

As a consequence of (3.2) we immediately get the minimum weight vectors of $H_{79}$ and $H_{78}$.

(3.3) Proposition. (1) $m\left(H_{79}\right)=m\left(H_{78}\right)=6$.

(2) $W_{6}\left(H_{79}\right)=W_{6}\left(H_{78}\right)$ is the set of all $\beta$-hexads, $\beta \in \Omega$.

(3) $\left|W_{6}\left(H_{79}\right)\right|=\left|W_{6}\left(H_{78}\right)\right|=3,850$.

Proof. From (2.7) we have $H_{78} \leqslant H_{79} \leqslant H_{99}=\{x \in F \Omega \mid w(x)$ even $\}$. Since $H_{78}$ is generated by all $\beta$-hexads, we also have $m\left(H_{78}\right) \leqslant 6$. Now suppose $0 \neq x \in H_{79}$ and $w(x) \leqslant 6$. Since $H_{79}$ is mapped by $v$ onto $H_{1}=F \mathbb{1}$ it follows $w(x v) \in\{0,100\}$. From (3.2) follows that $w(x)=6$, hence (1) holds. Since $\mathcal{G}_{100}$ is triangle-free there exist $x_{1} \in \Phi_{22}$ and $x_{2} \in W_{4}(F \Omega)$ such that $x=x_{1}+x_{2}$, hence $x v=x_{1} v+x_{2} v$. It follows by (3.2) that $w(x v) \leqslant w\left(x_{1} v\right)+w\left(x_{2} v\right) \leqslant 32+64=96<100$, thus necessarily $x v=0$, so $x \in \operatorname{Ker} v=H_{78}$. So we also have $x_{1} v=x_{2} v$ and $32=w\left(x_{1} v\right)=w\left(x_{2} v\right)$, hence $x_{2} \in \Phi_{41}$ according to (3.2). Consequently there exist distinct $\gamma, \beta \in \Omega$ such that $\operatorname{supp}\left(x_{2}\right) \subseteq \Delta(\beta) \cap \Delta(\gamma)$. Since $m\left(H_{78}\right)=6$ and $y=\Delta(\beta) \Delta(\gamma)$ (written with pointwise multiplication in $F \Omega$ ) is a $\beta$-hexad belonging to $H_{78}$ we have necessarily $x=y$, so also (2) holds. (3) follows from (2).

We shall also need a classification of weight 8 vectors in $H_{78}$. For this purpose and for later use it is convenient to introduce the following notation for vectors $x \in F \Omega$ and $0 \leqslant i \leqslant 22$ :

$$
\Lambda_{i}(x)=\{\beta \in \Omega|| \operatorname{supp}(x) \cap \Delta(\beta) \mid=i\} \text { and } \lambda_{i}(x)=\left|\Lambda_{i}(x)\right| .
$$

Call two vectors $x, y \in F \Omega \mathcal{G}_{100}$-disjoint iff $\operatorname{supp}(x) \cap \operatorname{supp}(y)=\emptyset$ and $\mathcal{G}_{100}$ has no edge joining a vertex in $\operatorname{supp}(x)$ and a vertex in $\operatorname{supp}(y)$. 
(3.4) Proposition. (1) $w_{8}\left(H_{78}\right)=119,625$.

(2) $W_{8}\left(H_{78}\right): G=\left\{\Phi_{81}, \Phi_{82}\right\}$ where $\left|\Phi_{81}\right|=33,000$ and $\left|\Phi_{82}\right|=86,625$. $\Phi_{81}$ is the set of $\beta$-octads, $\beta \in \Omega$. The elements of $\Phi_{82}$ are called skew octads; they are sums of two $\mathcal{G}_{100}$-disjoint vectors in $\Phi_{49}$ and any such sum is a skew octad.

(3) The stabilizer in $G$ of a skew octad is a Sylow 2-subgroup of $G$.

Proof. (1) and (3) are straightforward consequences of (2). Let $\Phi_{81}$ denote the set of all $\beta$-octads, $\beta \in \Omega$. Then clearly $\left|\Phi_{81}\right|=100 \cdot 330=33,000$, and $\Phi_{81}$ is a $G$-orbit in $W_{8}\left(H_{78}\right)$. Let $\Phi_{82}:=W_{8}\left(H_{78}\right) \backslash \Phi_{81}$ and call the elements of $\Phi_{82}$ skew octads.

Let $z$ be a skew octad. We set $\lambda_{i}=\lambda_{i}(z)$. Counting the edges of the Higman-Sims graph between then vertices in $\operatorname{supp}(z)$ and $\Omega$ gives the equations

$$
\lambda_{0}+\lambda_{2}+\lambda_{4}+\lambda_{6}+\lambda_{8}=100 \text { and } 2 \lambda_{2}+4 \lambda_{4}+6 \lambda_{6}+8 \lambda_{8}=8 \cdot 22=176 .
$$

(i) $\lambda_{8}=\lambda_{6}=0$.

For, if $\lambda_{8} \neq 0$ then $z$ would be a $\beta$-octad for some $\beta \in \Lambda_{8}(z)$ against the choice of $z$. If $\lambda_{6} \neq 0$ there existed a $\gamma \in \Lambda_{6}(z)$; taking the trace of $z v$ on $\Delta(\gamma)$ we see that $z v \neq 0$ against $z \in H_{78}=\operatorname{Ker} v$.)

(ii) The Higman-Sims graph does not induce the null graph on $\operatorname{supp}(z)$, i.e. $\operatorname{supp}(z) \nsubseteq$ $\Lambda_{0}(z)$.

Otherwise from (1.8) we deduce that for every $\xi \in \operatorname{supp}(z)$ the set $\Delta(\xi)$ is the disjoint union of te 10-set $\Delta(\xi) \cap \Lambda_{4}(z)$ and the 12-set $\Delta(\xi) \cap \Lambda_{2}(z)$. Consequently $\lambda_{4}=(8 \cdot 10) / 4=20, \lambda_{2}=(8 \cdot 12) / 2=48$ and $\lambda_{0}=32$. We consider the $\mathcal{G}_{100}$-edges between $\Lambda_{4}(z)$ and $\operatorname{supp}(z)$. Call a subset of $\operatorname{supp}(z)$ hexadic iff it is contained in the support of a $\beta$-hexad, $\beta \in \Omega$. Any 3 -subset $T$ of $\operatorname{supp}(z)$ is contained in exactly one $\beta$-hexad where $\beta \in \Lambda_{4}(z)$ since $\Delta(\beta) \cap \operatorname{supp}(z) \geqslant 3$; therefore $T$ is contained in exactly one hexadic 4-subset $\Delta(\beta) \cap \Delta(\gamma)$ where $\beta, \gamma \in \Lambda_{4}(z)$. Any hexadic 4-subset contains exactly 4 distinct 3 -subsets. Double counting now gives for the number $h$ of all hexadic 4 -subsets of $\operatorname{supp}(z)$ that $4 h=\left(\begin{array}{l}8 \\ 3\end{array}\right) \cdot 1=56$, so $h=14$. For each hexadic 4-subset $H=\Delta\left(\beta_{H}\right) \cap \Delta\left(\gamma_{H}\right)$ there are distinct $\beta_{H}, \gamma_{H} \in \Lambda_{4}(z)$ such that $\Delta\left(\beta_{H}\right) \cap \operatorname{supp}(z)=\Delta\left(\gamma_{H}\right) \cap \operatorname{supp}(z)=H$; moreover the map $H \mapsto\left\{\beta_{H}, \gamma_{H}\right\}$ has the property that $\left\{\beta_{H}, \gamma_{H}\right\} \cap\left\{\beta_{H^{\prime}}, \gamma_{H^{\prime}}\right\}=\emptyset$ if $H \neq H^{\prime}$. So we may deduce the absurdity $28=2 \cdot 14 \leqslant 20$. Therefore the Higman-Sims graph does not induce the null graph on $\operatorname{supp}(z)$.

(iii) $\operatorname{supp}(z) \cap \Lambda_{4}(z)=\emptyset$.

If there were a $\beta \in \operatorname{supp}(z) \cap \Lambda_{4}(z)$ we would obtain a contradiction from (1.5) by considering the trace of $z v$ on $\Delta(\beta)$. 
(iv) From (ii) and (iii) it follows that $\operatorname{supp}(z) \subseteq \Lambda_{0}(z) \cup \Lambda_{2}(z)$ and $\Lambda_{2}(z) \cap \operatorname{supp}(z) \neq \emptyset$. Without loss we may assume that $\alpha \in \Lambda_{2}(z) \cap \operatorname{supp}(z)$. From (1.5) we now derive that $z$ is the sum of two vectors in $\Phi_{49}$ which are $\mathcal{G}_{100}$-disjoint. Let $z=x+y$ be the sum of two $\mathcal{G}_{100}$-disjoint vectors $x, y \in \Phi_{49}$. From (1.5) we infer that $\operatorname{supp}(z v) \cap$ $\Delta(\beta)=\emptyset$ for all $\beta \in \operatorname{supp}(z)$. Since $\operatorname{supp}(z v) \subseteq \bigcup\{\Delta(\beta) \mid \beta \in \operatorname{supp}(z)\}$ it follows $z v=0$, hence $z \in H_{78}$ is a skew octad.

From the properties of $\mathcal{G}_{100}$ resp. $\mathcal{W}_{22}$ we deduce via (1.5) that the support of any element in $\Phi_{49}$ is contained in the support of exactly 6 distinct skew octads. Since the support of each skew octad contains exactly two different 4-sets which are supports of vectors in $\Phi_{49}$ we have

$$
\left|\Phi_{82}\right|=\left(6\left|\Phi_{49}\right|\right) / 2=3 \cdot 28,875=86,825
$$

which is also the index of a Sylow 2-subgroup in $G=\mathrm{HS}$. To complete the proof it now suffices to prove that the stabilizer $G_{z}$ in $G$ of a skew octad $z$ is a 2-group. Let $K$ be the largest normal subgroup of $G_{z}$ fixing every vertex in $\operatorname{supp}(z)$. Then $G_{z} / K$ is isomorphic to a subgroup of $Z_{2}$ wr $D_{8}$, hence $\left|G_{z}: K\right|$ is a power of 2 . But it is straightforward from the definition of the Higman-Sims graph that $K$ is a 2-group (see $(1.5)\left(\right.$ iii)). Therefore $G_{z}$ is a Sylow 2-subgroup and $\Phi_{82}$ is a $G$-orbit. The proof is complete.

Remark. The arguments in the preceding proofs show that there are precisely $\left|\Phi_{49}\right| / 7=4,125$ distinct elements $u \in W_{28}(F \Omega)$ such that $u$ is the sum of 7 pairwise $\mathcal{G}_{100^{-}}$ disjoint elements of $\Phi_{49}$. Of course, such a $u$ does not belong to $H_{79}$ since $u$ is congruent to an element of $\Phi_{49}$ modulo $H_{78}$. In fact $u v=x v$ for any $x \in \Phi_{49}$ with $\operatorname{supp}(x) \subseteq \operatorname{supp}(u)$ and $\operatorname{supp}(x) \subseteq \operatorname{supp}(u)$ holds for any $x \in \Phi_{49}$ such that $u v=x v$.

\section{(3.5) Theorem.}

The weight distribution of $H_{21}$ and the orbits of $G=\mathrm{HS}$ in $H_{21}$ are as described in the following table.

\begin{tabular}{|c|r|l|}
\hline$i$ & $w_{i}\left(H_{21}\right)$ & length of $G$-orbits in $W_{i}\left(H_{21}\right)$ \\
\hline $0 / 100$ & 1 & 1 \\
$32 / 68$ & 3,850 & 3,850 \\
$36 / 64$ & 4,125 & 4,125 \\
$40 / 60$ & 92,400 & 15,400 and 77,000 \\
$44 / 56$ & 347,600 & 1,100 and 346,500 \\
$48 / 52$ & 600,600 & 231,000 and 369,600 \\
\hline
\end{tabular}

In particular $m\left(H_{21}\right)=32$ and $G$ has precisely 18 orbits in $H_{21}$.

Proof. Since $\mathbb{1} \in H_{21}$ vectors and also $G$-orbits occur in complementary pairs belonging to weights $i$ and $100-i$. From (2.7) we infer that $x v \in H_{21}$ for all $x \in H_{99}$, therefore $x v \in H_{21}$ for all $x \in \Phi_{2 j} \cup \Phi_{4 k}$ by (3.2). We recall that $x v=y v$ if and only if $x+y \in H_{78}=\operatorname{Ker} v$. 
Of course, $v$ maps $G$-orbits to $G$-orbits.

It immediately follows that $\Psi_{1}=\left\{x v \mid x \in \Phi_{21}\right\}$ is a $G$-orbit in $W_{44}\left(H_{21}\right)$ of length $\left|\Psi_{1}\right|=$ 1,100 and that $\Psi_{2}=\left\{x v \mid x \in \Phi_{22}\right\}$ is a $G$-orbit in $W_{32}\left(H_{21}\right)$ of length $\left|\Psi_{2}\right|=3,850$. In counting with the orbits $\Phi_{4 j}$ we have to take care of multiple preimages when using Propositions (3.3) and (3.4). From Proposition (3.4) and the remark following its proof we infer that $\Psi_{3}=\left\{x v \mid x \in \Phi_{49}\right\}$ has exactly $\left|\Phi_{49}\right| / 7=4,125$ elements and by (3.2) we have $\Psi_{3} \subseteq W_{64}\left(H_{21}\right)$. The support of any skew octad contains also exactly 16 distinct 4 -subsets of type $\bullet-\bullet \quad \bullet \quad$ which are supports of vectors in $\Phi_{44}$. From (3.1) it hence follows that the support of any $x \in \Phi_{44}$ is contained in the support of exactly one skew octad. An elementary consideration shows that for any $x \in \Phi_{44}$ there exist also two distinct $\beta$-hexads $z, \beta \in \Omega$, such that $z=x+y$ where $y \in \Phi_{44}$. Hence from (3.3) and (3.4) it follows that $\Psi_{6}=\left\{x v \mid x \in \Phi_{44}\right\}$ has exactly $\left|\Phi_{44}\right| / 4=346,500$ elements and we have $\Psi_{6} \subseteq W_{44}\left(H_{21}\right)$ by $(3.2)$.

The support of every skew octad contains 32 distinct 4 -subsets of type $\bullet-\bullet-\bullet \bullet$ which are supports of vectors in $\Phi_{46}$. Hence the support of any $x \in \Phi_{46}$ is contained in the support of exactly 3 distinct skew octads. From (3.3) and (3.4) it now follows that $\Psi_{7}=\left\{x v \mid x \in \Phi_{46}\right\}$ has exactly $\left|\Phi_{46}\right| / 4=231,000$ elements and we have $\Psi_{7} \subseteq W_{48}\left(H_{21}\right)$ by $(3.2)$.

From (3.3) and (3.4) it also follows that $\Psi_{8}=\left\{x v \mid x \in \Phi_{47}\right\}$ has exactly $\left|\Phi_{47}\right|=369,600$ elements and from (3.2) we have $\Psi_{8} \subseteq W_{52}\left(H_{21}\right)$.

Taking into account that $\operatorname{dim} H_{21}=21$ the remarks at the beginning of the proof now show that the weight distribution of $H_{21}$ and the $G$-orbits in $H_{21}$ are as asserted in the theorem.

In order to determine the weight distribution of $H_{23}$ and its remaining subcodes it will be useful to get some information about the projection of $H_{23}$ to the neighborhoods $\Delta(\beta), \beta \in \Omega$. Since $G$ acts transitively on $\Omega$ we may restrict our attention to $\Delta(\alpha)=P_{22}$. We recall from Section 1 that $G_{12}$ denotes the shortened Golay code, $G_{10}=G_{12}^{\perp}$ its dual and $G_{11}, G_{11}^{\prime}$ and $G_{11}^{\prime \prime}$ are the subcodes between $G_{10}$ and $G_{12}$ where $G_{11}=G_{10}+F \mathbb{1}_{22}$ is generated by all hexads of $\mathcal{W}_{22}$.

\section{(3.6) Proposition.}

Let $p_{\alpha}: x \mapsto x \Delta(\alpha)$ denote the coordinate restriction of $H_{23}$ to $\Delta(\alpha)=P_{22}$ and let $K=\operatorname{Ker} p_{\alpha}$. Then the following hold:

(1) The sequence $0 \longrightarrow K \stackrel{\text { incl }}{\longrightarrow} H_{23} \stackrel{p_{\alpha}}{\longrightarrow} G_{12} \longrightarrow 0$ is exact.

(2) $K$ is contained in $H_{22}$, but not in $H_{21}$. $p_{\alpha}$ maps $H_{21}$ and $H_{22}$ onto $G_{11}$ and $H_{22}^{\prime}, H_{22}^{\prime \prime}, H_{23}$ onto $G_{12}$.

(3) $K$ has the weight distribution

\begin{tabular}{|c||c|c|c|c|}
\hline$i$ & $0 / 78$ & $22 / 56$ & $32 / 46$ & $38 / 40$ \\
\hline$w_{i}(K)$ & 1 & 22 & 231 & 770 \\
\hline
\end{tabular}

Proof. Since $H_{22}$ is generated by all adjacency vectors $\Delta(\beta), \beta \in \Omega$, it follows from (1.3) that $p_{\alpha}$ maps $H_{22}$ onto $G_{11}$. Furthermore $x(m) p_{\alpha}=m \in G_{12} \backslash G_{11}$ for any heptad $m$ of 
$\mathcal{W}_{22}$. Therefore $p_{\alpha}$ maps $H_{23}$ onto $G_{12}$ and (1) is established. Since $H_{22}$ is mapped by $p_{\alpha}$ onto a proper subspace of $G_{12}, K$ must be contained in $H_{22}$. Evidently $K$ contains the vector $\mathbb{1}+\Delta(\alpha)$ of weight $78 \not \equiv 0 \bmod 4$. Hence $K$ is not contained in $H_{21}$. The rest of (2) now easily follows. It remains to determine the weight distribution of $K$. We use the fact that $K \subseteq H_{22}=\operatorname{Im} v$. If $x v=y v$ then $(x+y) v=0$, i.e. $x+y \in \operatorname{Ker} v=H_{78}$. From this remark it follows by (3.2) that $K$ contains 22 vectors $\beta v=\Delta(\beta), \beta \in P_{22}$, of weight $22,\left(\begin{array}{c}22 \\ 2\end{array}\right)=231$ vectors $x v$ of weight 32 where $\operatorname{supp}(x)$ is a 2 -subset of $P_{22}$ and $\left(\begin{array}{c}22 \\ 3\end{array}\right) / 2=770$ vectors $y v$ of weight 28 where $\operatorname{supp}(y)$ is a 3 -subset of $P_{22}$. Since $\mathbb{1}+\Delta(\alpha)$ is the only vector in $K$ of weight 78 , assertion (3) now follows from $\operatorname{dim} K=11$.

\section{(3.7) Corollary.}

Let $x \in H_{22}$ and $\beta \in \Omega$. Then $w(x \Delta(\beta)) \in\{0,6,8,10,12,14,16,22\}$.

Proof. The assertion follows from (3.6) and (1.2) since $G$ acts transitively on $\Omega$.

\section{(3.8) Lemma.}

Let $u \in \Phi_{49}$. Then $G_{u}$ induces the dihedral group $D_{8}$ on the 4 vertices in $\operatorname{supp}(u)$.

Proof. The largest subgroup of $G_{u}$ fixing every vertex in $\operatorname{supp}(u)$ has index 8 in $G_{u}$ by (3.1).

\section{(3.9) Proposition.}

Let $x \in W_{36}\left(H_{21}\right)$ and $\bar{x}=x+\mathbb{1}$ its complementary vector.

Then $\Omega: G_{x}=\left\{\Lambda_{6}(x), \Lambda_{14}(x), \Lambda_{8}(x)\right\}$ where $\operatorname{supp}(x)=\Lambda_{6}(x) \cup \Lambda_{14}(x), \operatorname{supp}(\bar{x})=\Lambda_{8}(x)$ and $\lambda_{6}(x)=8, \lambda_{14}(x)=28, \lambda_{8}(x)=64$. The matrix of the Higman-Sims graph with respect to $G_{x}$ is

$$
\left[\begin{array}{ccc}
0 & 14 & 8 \\
4 & 2 & 16 \\
1 & 7 & 14
\end{array}\right] .
$$

Moreover, $\left(\sum \Lambda_{6}(x)\right) v=\bar{x}$ holds.

Proof. From (3.5) and (3.2) it follows that there exists a $u_{0} \in \Phi_{49}$ such that $u_{0} v=x$, and one easily checks that $u_{0} \cap \operatorname{supp}(\bar{x})=\emptyset$. From the remark following the proof of (3.4) we infer that the union of all $\operatorname{supp}(u), u \in \Phi_{49}$ such that $u v=x$, forms a set $B$ of cardinality 28 , disjoint from $\operatorname{supp}(\bar{x})$ and therefore contained in $\operatorname{supp}(x)$.

Clearly $\left(\sum B\right) v=\bar{x}$ holds. Set $A=\operatorname{supp}(x) \backslash B$ and $\Gamma=\operatorname{supp}(\bar{x})$. Obviously, $A, B$ and $\Gamma$ are fixed by $G_{x}$; we show that $G_{x}$ acts transitively on $A, B$ and $\Gamma$. The elements of order 7 in $G$ have exactly 2 fixed-points in $\Omega$. Since $\left|G_{x}\right|$ does not divide $\left|M_{22}\right|$ the subgroup $G_{x}$ has no fixed-point in $\Omega$. From 7||$G_{x} \mid$ we readily conclude that $G_{x}$ acts 2transitively on $A$ and that $G_{x}$ permutes transitively the 7 elements of $\Phi_{49}$ whose supports are contained in $B$. Let $u \in \Phi_{49}$ be such that $\operatorname{supp}(u)$ is contained in $B$. Since $v$ is a $G$-homomorphism $G_{u} \leqslant G_{u v}=G_{\bar{x}}=G_{x}$. So from (3.8) it follows that $G_{x}$ acts transitively on $B$.

Let $G_{\dot{u}}$ denote the largest subgroup of $G_{u}$ fixing every vertex in $\operatorname{supp}(u)$. From the fundamental properties of the Higman-Sims graph it follows that $G_{\dot{u}}$ has exactly 4 orbits 
of length 16 which are conjugate under $G_{u}$ by (3.8). Hence $G_{u}$ acts transitively on $\Gamma$. Since $G_{x}$ acts doubly-transitively on $A$ the Higman-Sims graph induces on $A$ the null graph. Moreover, the preceding discussion shows that for $\beta \in B$ we have $|\Delta(\beta) \cap B|=2$ and $|\Delta(\beta) \cap \Gamma|=16$. It easily follows that the matrix of the Higman-Sims graph with respect to $G_{x}$ is as asserted; in particular, $A=\Lambda_{14}(x), B=\Lambda_{6}(x)$ and $\Gamma=\Lambda_{8}(x)$.

(3.10) Proposition. (1) $w_{i}\left(H_{22}\right)=0$ for $0<i<32$ and $i \neq 22,30$.

(2) $w_{22}\left(H_{22}\right)=100$ and $w_{30}\left(H_{22}\right)=1,100$.

(3) $W_{22}\left(H_{22}\right)=\{\Delta(\gamma) \mid \gamma \in \Omega\}$ is a $G$-orbit; $G_{\Delta(\gamma)}=G_{\gamma}$ for $\gamma \in \Omega$.

(4) $W_{30}\left(H_{22}\right)$ is a $G$-orbit. For $x \in W_{30}\left(H_{22}\right)$ we have $\Omega: G_{x}=\left\{\Lambda_{8}(x), \Lambda_{6}(x)\right\}$ where $\operatorname{supp}(x)=\Lambda_{8}(x), \operatorname{supp}(x+\mathbb{1})=\Lambda_{6}(x)$. In particular, $\lambda_{8}(x)=30$ and $\lambda_{6}(x)=70$.

Proof. $H_{22} \leqslant H_{99}=\langle\mathbb{1}\rangle^{\perp}$ implies that $w_{i}\left(H_{22}\right)=0$ for every odd $i$. Let $0 \neq x \in H_{22}$ such that $w(x)<32$. From (3.5) it follows that either $w(x)=30$ or $w(x) \leqslant 26$. Set $\lambda_{j}=\lambda_{j}(x)$.

(i) Suppose that $w(x) \neq 30$. We claim that in this case $w(x)=22$ and $x=\Delta(\gamma)$ for some $\gamma \in \Omega$.

Assume the contrary. We may choose $x$ as a counterexample of minimal weight. If $\lambda_{22} \neq 0$ there would exist a $\gamma \in \Lambda_{22}(x)$ and then $w(x) \geqslant 22$ and $w(x+\Delta(\gamma)) \leqslant 4$, consequently $x+\Delta(\gamma)=0$ and $x=\Delta(\gamma)$ by (3.3), contrary to the assumption. If $\lambda_{0} \neq 0$ we would have also $\lambda_{22} \neq 0$ by (3.6). Hence we may suppose that $\lambda_{22}=0=\lambda_{0}$. We have $w(x+\Delta(\beta)) \geqslant w(x)$ for all $\beta \in \Omega$. For, otherwise, $x+\Delta(\beta)=\Delta(\gamma)$ by the minimal choice of $x$, so $x=\Delta(\beta)+\Delta(\gamma)=(\beta+\gamma) v$ which contradicts (3.5) because of $\beta+\gamma \in \Phi_{21} \cup \Phi_{22}$, see (3.2). For $\beta \in \Lambda_{j}(x)$ we therefore have $w(x)+22-2 j \geqslant w(x)$ and $j \leqslant 11$ follows. Therefore $\lambda_{j} \neq 0$ implies $j \in\{6,8,10\}$ by (3.7). Counting the edges of the Higman-Sims graph between $\operatorname{supp}(x)$ and $\Omega$ now yields the equations

$$
22 w(x)=6 \lambda_{6}+8 \lambda_{8}+10 \lambda_{10} \text { and } 100=\lambda_{6}+\lambda_{8}+\lambda_{10} .
$$

From these equations we deduce $11 w(x)=300+\lambda_{8}+2 \lambda_{10} \geqslant 300$ and $w(x)>27$, again a contradiction.

So the claim is proved and (1) holds. By the arguments above we also have obtained that $W_{22}\left(H_{22}\right)$ is precisely the set of all adjacency vectors $\Delta(\beta), \beta \in \Omega$. Hence (3) holds and $w_{22}\left(H_{22}\right)=100$.

(ii) Suppose $w(x)=30$. Our first claim is that $\lambda_{8}=30$ and $\lambda_{6}=70$.

Since $w_{8}\left(H_{22}\right)=0$ by (1) we have $\lambda_{22}=0$. From (3.6) we infer that $\lambda_{0}=0$. Furthermore, for all $\beta \in \Omega$ we have $w(x+\Delta(\beta)) \geqslant 30$ since otherwise $x+\Delta(\beta)=$ $\Delta(\gamma)$ for some $\gamma \in \Omega$ and $x \in H_{21}$ which is impossible. Therefore we have again 
$\lambda_{j} \neq 0$ only possibly for $j \in\{6,8,10\}$ by (3.7). We assert that also $\lambda_{10}=0$. For, if $\beta \in \Lambda_{10}(x)$ we had $w(x+\Delta(\beta))=32$, hence $x+\Delta(\beta)=\Delta(\gamma)+\Delta(\delta)$ for suitable $\gamma, \delta \in \Omega$ by (3.5) and (3.7), which entails that

$$
x=\Delta(\beta)+\Delta(\gamma)+\Delta(\delta)=(\beta+\gamma+\delta) v,
$$

a contradiction against (3.2). Counting edges now yields the equations

$$
6 \lambda_{6}+8 \lambda_{8}=22 \cdot 30=660 \text { and } \lambda_{6}+\lambda_{8}=100
$$

which have the unique solution $\lambda_{6}=70$ and $\lambda_{8}=30$.

(iii) For every $x \in W_{30}\left(H_{22}\right)$ and every $\beta \in \Lambda_{8}(x)$ we have $x+\Delta(\beta) \in W_{36}\left(H_{22}\right)=$ $W_{36}\left(H_{21}\right)$ which is a $G$-orbit of length 4,125 . On the other hand for every $z \in$ $W_{36}\left(H_{22}\right)=W_{36}\left(H_{21}\right)$ and $\gamma \in \Lambda_{14}(z)$ we have $z+\Delta(\gamma) \in W_{30}\left(H_{22}\right)$. Hence $W_{30}\left(H_{22}\right) \neq \emptyset$. We consider the incidence structure

$$
\mathcal{I}=\left(W_{30}\left(H_{22}\right), W_{36}\left(H_{22}\right), \mathrm{I}\right)
$$

where $\mathrm{I}=\left\{(x, z) \mid x \in W_{30}\left(H_{22}\right), z \in W_{36}\left(H_{22}\right)\right.$ and $\left.x+z \in W_{22}\left(H_{22}\right)\right\}$. It follows from (3.9) that $G$ acts transitively on I. Since $G$ acts transitively on $W_{36}\left(H_{21}\right)$ we conclude that also $W_{30}\left(H_{22}\right)$ is a $G$-orbit and that $G_{x}$ acts transitively on $\Lambda_{8}(x)$ for $x \in W_{36}\left(H_{22}\right)$. Double counting of I then gives

$$
w_{30}\left(H_{22}\right) \cdot 30=|\mathrm{I}|=w_{36}\left(H_{22}\right) \cdot 8=4,125 \cdot 8=33,000,
$$

hence $w_{30}\left(H_{22}\right)=1,100$. From (3.9) we may deduce that for $x \in W_{30}\left(H_{22}\right)$ we have $\Lambda_{8}(x) \cap \operatorname{supp}(x+\mathbb{1})=\emptyset$, hence $\Lambda_{8}(x)=\operatorname{supp}(x)$ and $\Lambda_{6}(x)=\operatorname{supp}(x+\mathbb{1})$. One checks by inspection that for $z \in W_{36}\left(H_{22}\right)$ and $\beta \in \Lambda_{14}(z)$ the subgroup $G_{z, \beta}$ has an orbit of length 14 in $\operatorname{supp}(z+\Delta(\beta)+\mathbb{1})$. It again follows by counting of edges that for $x \in W_{30}\left(H_{22}\right)$ the subgroup $G_{x}$ acts transitively on $\operatorname{supp}(x+\mathbb{1})=\Lambda_{6}(x)$.

Remark. It will be shown in (4.9) that $G_{x} \cong \Sigma_{8}$ for $x \in W_{30}\left(H_{22}\right)$.

The results we have yet obtained are sufficient to determine the weight distribution of the code $\mathrm{H}_{22}$ via the MacWilliams identities.

\section{(3.11) Theorem.}

The weight distribution of $H_{22}$ and the orbits of $G$ in $H_{22}$ are as described in Table 1. In particular, $G$ has precisely 34 orbits in $H_{22}$. Complementary vectors of weight 50 are in the same $G$-orbit.

Proof. Let $a_{i}=w_{i}\left(H_{22}\right)$ and $b_{i}=w_{i}\left(H_{78}\right)$. We recall that $H_{78}=H_{22}{ }^{\perp}$. Hence the families $\left(a_{i}\right)$ and $\left(b_{i}\right)$ are related to each other by the MacWilliams identities.

We have $H_{22} \leqslant H_{78} \leqslant H_{99}$; hence $a_{i}=b_{i}=0$ holds for every odd $i$. Since $\mathbb{1} \in H_{22}$ we have $a_{i}=a_{100-i}$ for all $i$. From (3.10) we have the information that

$$
a_{i}=0 \text { for } 0<i<32 \text { with the exceptions } a_{22}=100, a_{30}=1,100 .
$$




\begin{tabular}{|c|r|c|}
\hline$i$ & $w_{i}\left(H_{22}\right)$ & length of $G$-orbits in $W_{i}\left(H_{22}\right)$. \\
\hline $0 / 100$ & 1 & 1 \\
$22 / 78$ & 100 & 100 \\
$30 / 70$ & 1,100 & 1,100 \\
$32 / 68$ & 3,850 & 3,850 \\
$36 / 64$ & 4,125 & 4,125 \\
$38 / 62$ & 38,500 & 38,500 \\
$40 / 60$ & 92,400 & 15,400 and 77,000 \\
$42 / 58$ & 193,600 & 61,600 and 132,000 \\
$44 / 56$ & 347,600 & 1,100 and 346,500 \\
$46 / 54$ & 485,100 & 23,100 and 462,000 \\
$48 / 52$ & 600,600 & 231,000 and 369,600 \\
50 & 660,352 & 44,352 and 616,000 \\
\hline
\end{tabular}

Table 1: The weight distribution of $H_{22}$ and the orbits of $G$ in $H_{22}$.

Furthermore $a_{0}=a_{100}=1$ trivially holds, and from $(3.5)$ every $a_{i}$ with $i \equiv 0(\bmod 4)$ is known. So the only unknown weight numbers of $H_{22}$ with $i \leqslant 50$ are

$$
a_{34}, a_{38}, a_{42}, a_{46} \text { and } a_{50} .
$$

On the other hand we know from (3.3) and (3.4) the values

$$
b_{0}=1, b_{2}=b_{4}=0, b_{6}=3,850, b_{8}=119,625 .
$$

It follows from the general theory of MacWilliams identities that the weight distribution $\left(a_{i}\right)$ is uniquely determined by the known values of the $a_{i}$, and $b_{j}$, see e.g. [28]. Explicit calculations yield the values asserted in the theorem.

It remains to determine the $G$-orbits in $H_{22} \backslash H_{21}$. It is convenient to consider first the factor module $H_{22} / H_{1}$ which consists of pairs $\{x, x+\mathbb{1}\}=x+H_{1}$ of complementary vectors, $x \in H_{22}$. Because of $H_{79}=H_{21}^{\perp}$ we clearly have $H_{22} / H_{1} \cong_{F G} H_{100} / H_{79} \cong_{F G} H_{21}{ }^{*}$.

Since $F=\mathbb{F}_{2}$, by Lemma (1.2) $G$ has the same number of orbits in $H_{21}^{*}$ as in $H_{21}$. By (3.5) therefore $G$ has exactly 18 orbits in $H_{21} / H_{1}$. By (3.5) $G$ also has 9 orbits in $H_{21} / H_{1}$, so $G$ has exactly 9 orbits in $H_{22} / H_{1} \backslash H_{21} / H_{1}$.

For $x+H_{1} \in F \Omega / H_{1}$ we define the weight $w\left(x+H_{1}\right)=\{w(x), w(x+\mathbb{1})\}$. From (3.10) it follows that $G$ has in $H_{22} / H_{1}$ one orbit of elements of weight $\{22,78\}$ of length 100 and one orbit of elements of weight $\{30,70\}$ and length 1,100. From (3.1), (3.2), and (3.3) it follows that $G$ has in $H_{22} / H_{1}$ an orbit of elements of weight $\{38,62\}$ and length 38,500 . The weight distribution of $H_{22}$ which we have determined tells us that all remaining pairs $x+H_{1}$ have weight $\{i, 100-i\}$ where $i \in\{42,46,50\}$. From (3.1), (3.2) and (3.3) we also obtain that $G$ has in $H_{22} / H_{1}$ an orbit of elements of weight $\{42,58\}$ and length 61,600 and an orbit of elements of weight $\{46,54\}$ and length 23,100 . Since $a_{50} / 2=330,176$ is not a divisor of the order of $G$ we have at least two $G$-orbits of elements of weight $\{50,50\}$ in $H_{22} / H_{1}$. Since $G$ has exactly 9 orbits in $H_{22} / H_{1} \backslash H_{21} / H_{1}$ it follows that 
$G$ has precisely two more orbits: one of length 132, 000 consisting of elements of weight $\{42,58\}$ and one of length 462,000 consisting of elements of weight $\{46,54\}$. To complete the proof of the theorem we have to consider vectors of weight 50 . We claim that there are even only $2 G$-orbits in $W_{50}\left(H_{22}\right)$, i.e. that complementary vectors in $W_{50}\left(H_{22}\right)$ are in the same $G$-orbit. This assertion and the length of the two $G$-orbits in $W_{50}\left(H_{22}\right)$ are obtained in the following lemma.

\section{(3.12) Lemma.}

Let $P, Q$ be two distinct elements of $\Delta(\alpha)$ and $h, k, \ell \in \Delta_{2}(\alpha)$ such that

$$
Q \in h, Q \notin k \cup \ell, h \cap k=\emptyset, h \cap \ell \neq \emptyset \text { and } P \in k \cap \ell .
$$

Set $u=\alpha+P+Q+h, u_{1}=u+k$ and $u_{2}=u+\ell$ and let $50_{i}$ denote the $G$-orbit containing $x_{i}=u_{i} v(i=1,2)$. Then the following hold.

(1) $W_{50}\left(H_{22}\right)=50_{1} \cup 50_{2}$.

(2) $\left|50_{1}\right|=44,352$ and $\left|50_{2}\right|=616,000$.

Proof. Set $\bar{x}=u v$ and $x=\bar{x}+1$. By construction $u \in \Phi_{47}$ and from (3.2) it follows that $x \in 48_{2}$ where $48_{2}$ denotes the $G$-orbit in $W_{48}\left(H_{22}\right)$ of length 369,600 as above. Furthermore, we have $G_{x}=G_{\bar{x}}=G_{u} \cong \Sigma_{5}$ (by considering a hexad stabilizer). Elementary considerations show that $\Lambda_{10}(x)$ splits into a unique $G_{x}$-orbit $\Psi_{1}$ of length 6 and its complement $\Psi_{2}$ of cardinality 30 . Moreover, $k \in \Psi_{1}$ and $\ell \in \Psi_{2}$.

It is easy to show that $G_{x_{1}}$ acts transitively on $\operatorname{supp}\left(x_{1}\right)=\Lambda_{12}\left(x_{1}\right)$. We therefore can count the incidences in the incidence structure $\mathcal{I}_{1}=\left(48_{2}, 50_{1}, \mathrm{I}_{1}\right)$ where

$$
\mathrm{I}_{1}=\left\{(w, z) \mid w \in 48_{2}, z \in 50_{1} \text { and } w+\Delta(\gamma)=z \text { such that }\left|\gamma G_{w}\right|=6\right\},
$$

obtaining $369,600 \cdot 6=\left|50_{1}\right| \cdot 50$, hence $\left|50_{1}\right|=44,352$. (It easily follows that $G_{x_{1}}$ acts transitively also on $\operatorname{supp}\left(\overline{x_{1}}\right)=\Lambda_{10}\left(x_{1}\right)$ where $\overline{x_{1}}=x_{1}+\mathbb{1}$ and that $x_{1}$ and $\overline{x_{1}}$ are in the same $G$-orbit.)

The preceding arguments also show that $50_{2} \neq 50_{1}$. Since $G$ has exactly 2 orbits of vectors of weight $\{50,50\}$ in $H_{22} / H_{1}$ we have either $\left|50_{2}\right|=616,000$ or $\left|50_{2}\right|=308,000$. In order to decide this alternative we consider $\Lambda_{16}\left(x_{2}\right)$ and we find $\left|\Lambda_{16}\left(x_{2}\right)\right|=2$ by direct examination. If $\gamma \in \Lambda_{16}\left(x_{2}\right)$ then $x_{2}+\Delta(\gamma) \in W_{40}\left(H_{22}\right)=40_{1} \cup 40_{2}$ where $40_{1}$ denotes the $G$-orbit of length 15,400 and $40_{2}$ denotes the $G$-orbit of length 77,000, see (3.11). From (3.5) we infer that for $y \in 40_{2}$ the set $\Lambda_{6}(y)$ is a $G_{y}$-orbit of length 16 and that $\Lambda_{6}(z)=\emptyset$ for any $z \in 40_{1}$. Therefore we consider the incidence structure

$$
\mathcal{I}_{2}=\left(40_{1}, 50_{2}, \mathrm{I}_{2}\right) \text { where } \mathrm{I}_{2}=\left\{(w, z) \mid w \in 40_{1}, z \in 50_{2} \text { and } w+\Delta(\gamma)=z \text { for some } \gamma\right\}
$$

Counting incidences gives $\left|40_{1}\right| \cdot 16=\left|50_{2}\right| \cdot 2$, hence $\left|50_{2}\right|=\left|40_{1}\right| \cdot 8=616,000$ which completes the proof of the lemma. 
The argumentation in (3.12) (and in (3.9)) may be extended to all $G$-orbits in $H_{22}$ in order to obtain results concerning the "set connectivity" in the Higman-Sims graph. We list the results in the following proposition, omitting proofs which tend to be tedious calculations.

$G$-orbits consisting of vectors of weight $k$ are denoted by $k$ or $k_{1}, k_{2}$ using the convention that $\left|k_{1}\right|<\left|k_{2}\right|$. We give the relevant information in the graph matrix of the $G$-orbits in $H_{22}$ which is defined as follows:

The row and colomn indices are the $G$-orbits in $H_{22}$. The entry $m ; n$ belonging to the ordered index pair $(k, \ell)$ tells that there are exactly $m$ vertices $\beta \in \Omega$ such that $x+\Delta(\beta) \in \ell$ for any $x \in k$ and that there are exactly $n$ vertices $\gamma \in \Omega$ such that $y+\Delta(\gamma) \in k$ for any $y \in \ell$. The entry $0 ; 0$ is replaced by -- for better readability.

We give the graph matrix in a reduced form from which the complete matrix is easily derived by considering complementary vectors.

(3.13) Proposition.

The graph matrix of the $G$-orbits in $H_{22}$ is given by the following submatrix.

\begin{tabular}{|c|ccccccccc|}
\hline & 22 & 30 & 38 & $42_{1}$ & $42_{2}$ & $46_{1}$ & $46_{2}$ & $50_{1}$ & $50_{2}$ \\
\hline 0 & $100 ; 1$ & -- & -- & -- & -- & -- & -- & -- & -- \\
32 & $2 ; 77$ & -- & $60 ; 6$ & $32 ; 2$ & -- & -- & -- & -- & -- \\
68 & -- & -- & -- & -- & -- & $6 ; 1$ & -- & -- & -- \\
36 & -- & $8 ; 30$ & -- & -- & $64 ; 2$ & $28 ; 5$ & -- & -- & -- \\
$40_{1}$ & -- & -- & -- & $40 ; 10$ & -- & -- & $60 ; 2$ & -- & -- \\
$40_{2}$ & -- & $1 ; 70$ & $16 ; 32$ & -- & $24 ; 14$ & $6 ; 20$ & $36 ; 6$ & -- & $16 ; 2$ \\
$60_{2}$ & -- & -- & $1 ; 2$ & -- & -- & -- & -- & -- & $16 ; 2$ \\
$44_{1}$ & $2 ; 22$ & -- & -- & $56 ; 1$ & -- & -- & -- & -- & -- \\
$56_{1}$ & -- & -- & -- & -- & -- & $42 ; 2$ & -- & -- & -- \\
$44_{2}$ & -- & -- & $4 ; 36$ & $8 ; 45$ & $16 ; 42$ & -- & $32 ; 24$ & -- & $32 ; 18$ \\
$56_{2}$ & -- & -- & -- & -- & -- & -- & $8 ; 6$ & -- & $32 ; 18$ \\
$48_{1}$ & -- & -- & $4 ; 24$ & $8 ; 30$ & -- & -- & $32 ; 16$ & -- & $32 ; 12$ \\
$52_{1}$ & -- & -- & -- & -- & $8 ; 14$ & $4 ; 40$ & $12 ; 6$ & -- & $32 ; 12$ \\
$48_{2}$ & -- & -- & -- & -- & $10 ; 28$ & $2 ; 32$ & $30 ; 24$ & $6 ; 50$ & $30 ; 18$ \\
$52_{2}$ & -- & -- & -- & $2 ; 12$ & -- & -- & $20 ; 16$ & $6 ; 50$ & $30 ; 18$ \\
\hline
\end{tabular}

Proof. Omitted.

From (3.13) (or other elementary considerations) we can derive for any $G$-orbit $k$ the minimum weight $m(k)$ of a vector $u \in F \Omega$ such that $u v \in k$.

\section{(3.14) Corollary.}

The values $m(k)$ for the $G$-orbits $k$ in $H_{22}$ are given in Table 2. Moreover, every $x \in F \Omega$ is congruent to a vector of weight at most 8 modulo $H_{78}$ and to a vector of weight at most 5 modulo $H_{79}$. Hence every coset leader of $H_{78}$ has weight at most 8.

Now we may use the MacWilliams transformation to obtain the weight distributions of the codes $H_{78}=H_{22}{ }^{\perp}$ and $H_{79}=H_{21}{ }^{\perp}$. (This computation has been carried out first 


\begin{tabular}{|cc|cc|}
\hline$k$ & $m(k)$ & $k$ & $m(k)$ \\
\hline 0 & 0 & 100 & 8 \\
32 & 2 & 68 & 6 \\
36 & 6 & 64 & 4 \\
$40_{1}$ & 4 & $60_{1}$ & 4 \\
$40_{2}$ & 4 & $60_{2}$ & 4 \\
$44_{1}$ & 2 & $56_{1}$ & 6 \\
$48_{1}$ & 4 & $52_{1}$ & 6 \\
$48_{2}$ & 6 & $52_{2}$ & 4 \\
& & & \\
22 & 1 & 78 & 7 \\
30 & 5 & 70 & 5 \\
38 & 3 & 62 & 5 \\
$42_{1}$ & 3 & $58_{1}$ & 7 \\
$42_{2}$ & 5 & $5_{2}$ & 5 \\
$46_{1}$ & 5 & $54_{1}$ & 3 \\
$46_{2}$ & 5 & $54_{2}$ & 5 \\
$50_{1}$ & 5 & $50_{2}$ & 5 \\
\hline
\end{tabular}

Table 2: The values $m(k)$ for the $G$-orbits $k$ in $H_{22}$.

in 1980 by F.H Florian at the Rechenzentrum of Tübingen University using the ALDES program for computing with large numbers; nowadays it is easy to obtain the result by suitable computer algebra software like e.g. GAP [10].)

\section{(3.15) Proposition.}

The weight distributions of $H_{78}$ and of $H_{79}$ are as given in Table 3.

Proof. The assertion follows from (3.11) and (3.5) by the MacWilliams transformation.

Our next purpose is to determine the weight distribution and the $G$-orbits of the codes $H_{22}^{\prime}$ and $H_{22}^{\prime \prime}$. These codes are conjugate under the permutation group $\bar{G} \cong \operatorname{Aut}(G)$; hence they have the same weight distribution and the same $G$-orbit structure so that they can be discussed simultaneously. Since $H_{23}=H_{22} \cup H_{22}^{\prime} \cup H_{22}^{\prime \prime}$ we obtain all information also about $H_{23}$.

At first, we complete the classification of vectors in $W_{8}\left(H_{79}\right)$.

\section{(3.16) Lemma.}

For every $x \in H_{79} \backslash H_{78}$ and every $\beta \in \Omega w(x \Delta(\beta))=|\operatorname{supp}(x) \cap \Delta(\beta)|$ is odd.

Proof. This is an immediate consequence of (2.7)(8).

\section{(3.17) Proposition.}

$W_{8}\left(H_{79}\right) \backslash W_{8}\left(H_{78}\right)$ consists precisely of all vectors $\beta+m$ where $m$ is a $\beta$-heptad. In 


\begin{tabular}{|c|r|r|}
\hline$i$ & $w_{i}\left(H_{78}\right)$ & $w_{i}\left(H_{79}\right)$ \\
\hline $0 / 100$ & 1 & 1 \\
$6 / 94$ & 3,850 & 3,850 \\
$8 / 92$ & 119,625 & 154,825 \\
$10 / 90$ & $8,625,540$ & $16,387,140$ \\
$12 / 88$ & $504,741,475$ & $1,003,835,875$ \\
$14 / 86$ & $21,060,732,550$ & $42,133,634,950$ \\
$16 / 84$ & $641,604,305,375$ & $1,283,480,881,375$ \\
$18 / 82$ & $14,622,264,133,400$ & $29,244,271,163,800$ \\
$20 / 80$ & $255,578,801,503,795$ & $511,152,645,567,795$ \\
$22 / 78$ & $3,496,197,414,021,950$ & $6,992,403,401,202,750$ \\
$24 / 76$ & $38,040,184,865,580,975$ & $76,080,408,035,945,775$ \\
$26 / 74$ & $333,583,288,959,605,300$ & $667,166,480,352,256,500$ \\
$28 / 72$ & $2,383,620,258,950,558,925$ & $4,767,240,353,068,238,925$ \\
$30 / 70$ & $14,005,822,677,643,540,370$ & $28,011,646,019,961,809,810$ \\
$32 / 68$ & $68,193,674,451,079,227,050$ & $136,387,349,145,968,724,650$ \\
$34 / 66$ & $276,907,651,030,419,444,000$ & $553,815,299,356,210,253,600$ \\
$36 / 64$ & $942,804,612,331,379,390,725$ & $1,885,609,223,857,102,552,325$ \\
$38 / 62$ & $2,703,690,041,528,811,696,900$ & $5,407,380,102,022,140,311,300$ \\
$40 / 60$ & $6,554,715,235,199,646,035,290$ & $13,109,430,428,316,832,071,770$ \\
$42 / 58$ & $13,474,850,115,575,617,584,200$ & $26,949,700,280,870,672,877,000$ \\
$44 / 66$ & $23,545,377,618,939,915,393,150$ & $47,090,755,183,602,450,042,750$ \\
$46 / 64$ & $35,033,702,002,644,035,359,900$ & $70,067,404,105,299,389,919,900$ \\
$48 / 52$ & $44,444,350,668,327,576,562,750$ & $88,888,701,152,233,082,111,550$ \\
50 & $48,108,741,996,656,177,342,352$ & $96,217,484,223,130,147,456,656$ \\
\hline
\end{tabular}

Table 3: The weight distributions of $H_{77}$ and $H_{78}$.

particular $w_{8}\left(H_{79}\right)=w_{8}\left(H_{78}\right)+35,2000$ and $G$ has exactly two orbits $\Phi_{8}^{\prime}$ and $\Phi_{8}^{\prime \prime}$ of length 17,600 in $W_{8}\left(H_{79}\right) \backslash W_{8}\left(H_{78}\right)$. These $G$-orbits are conjugate under $\bar{G} \cong \operatorname{Aut}(G)$.

Choosing suitable notation we have $\Phi_{8}^{\prime} \subseteq H_{78}^{\prime}$ and $\Phi_{8}^{\prime \prime} \subseteq H_{78}^{\prime \prime}$.

Proof. Let $m$ be a $\beta$-heptad, $\beta \in \Omega$. Then we have $(\beta+m) v=\mathbb{1}$, as follows from (1.9). Hence $\beta+m \in W_{8}\left(H_{79}\right) \backslash H_{78}$. From (1.3) we infer that $G_{\alpha} \cong M_{22}$ has two orbits on the set of $\alpha$-heptads, the orbits being conjugate under the action of $\bar{G}_{\alpha} \cong \operatorname{Aut}\left(M_{22}\right)$. It follows that $G$ has two orbits $\Phi_{8}^{\prime}$ and $\Phi_{8}^{\prime \prime}$ of length 17,600 in $W_{8}\left(H_{79}\right) \backslash W_{8}\left(H_{78}\right)$ which are conjugate under $\bar{G} \cong \operatorname{Aut}(G)$, where $\Phi_{8}^{\prime}$ and $\Phi_{8}^{\prime \prime}$ consist of vectors $\beta+m$ where $m$ is a $\beta$-heptad.

If $m$ is an $\alpha$-heptad we have $\langle\alpha+m, x(m)\rangle=0$ for the Higman vector $x(m)=$ $\alpha+m+B_{1}(m)$, see (1.10) and (2.7). Hence we conclude from (2.7) that we may choose the notation so that $\Phi_{8}^{\prime} \subseteq H_{78}^{\prime}$ and $\Phi_{8}^{\prime \prime} \subseteq H_{78}^{\prime \prime}$. From (3.15) we have $w_{8}\left(H_{79}\right)=w_{8}\left(H_{78}\right)+$ 35,200 and the assertion follows.

However, it is easy to avoid the use of (3.15) in order to obtain the result. We give a 
sketch of a short direct proof:

Let $y \in W_{8}\left(H_{79}\right) \backslash H_{78}$ and let $k(y)=\max \{|\Delta(\xi) \cap \operatorname{supp}(y)| \mid \xi \in \operatorname{supp}(y)\}$. Then $k(y) \in\{1,3,5,7\}$ by (3.16). The possibility $k(y) \in\{1,3,5\}$ is ruled out by easy contradictions. Therefore $k(y)=7$ and it follows that $y=\beta+m$ for a vector $\mathrm{m}$ of weight 7 such that $\operatorname{supp}(m) \subseteq \Delta(\beta)$. Without loss we may assume $\beta=\alpha$; then we obtain from (1.3) that $m$ is a heptad in $\mathcal{W}_{22}$ from the fact that $\langle y, \Delta(\gamma)\rangle=1$ for every $\gamma \in \Delta_{2}(\alpha)$.

\section{(3.18) Lemma.}

Let $x \in H_{23} \backslash H_{22}$ and $\beta \in \Omega$. Then $w(x \Delta(\beta))=|\operatorname{supp}(x) \cap \Delta(\beta)| \in\{7,11,15\}$.

Proof. The assertion follows from (3.6) and (1.3) since $G$ acts transitively on $\Omega$.

\section{(3.19) Proposition.}

The code $H_{22}^{\prime}$ has minimum weight 32.

Proof. $H_{21}$ is the subcode of $H_{22}^{\prime}$ consisting of all vectors of weight divisible by 4 . Since 32 is the minimum weight of $H_{21}$ by (3.5) it suffices to show that $w_{i}\left(H_{22}^{\prime}\right)=0$ for all $0<i<32$. Let $x \in W_{i}\left(H_{22}^{\prime}\right)$ where $0<i \leqslant 32$. Let $\lambda_{j}=\lambda_{j}(x)$ for $0 \leqslant j \leqslant 22$. Counting the edges of the Higman-Sims graph between $\operatorname{supp}(x)$ and $\Omega$ gives by (3.18) the equations

$$
\begin{array}{cccc}
w(x) \cdot 22 & = & 7 \lambda_{7}+11 \lambda_{11}+15 \lambda_{15} \\
100 & =\lambda_{7}+\lambda_{11}+\lambda_{15} .
\end{array}
$$

It follows $w(x) \cdot 22=700+4 \lambda_{11}+8 \lambda_{15} \geqslant 700$ and $w(x)>31$, hence $w(x)=32$.

We are now in a position to obtain the weight distributions of $H_{22}^{\prime}$ and $H_{23}$ using the MacWilliams or Pless identities.

\section{(3.20) Theorem.}

The weight distribution of $H_{22}^{\prime}$ and the orbits of $G$ in $H_{22}^{\prime}$ are as described in the following table.

\begin{tabular}{|c|r|c|}
\hline$i$ & $w_{i}\left(H_{22}^{\prime}\right)$ & length of $G$-orbits in $W_{i}\left(H_{22}^{\prime}\right)$. \\
\hline $0 / 100$ & 1 & 1 \\
$32 / 68$ & 3,850 & 3,850 \\
$34 / 66$ & 5,600 & 5,600 \\
$36 / 64$ & 4,125 & 4,125 \\
$38 / 62$ & 38,500 & 38,500 \\
$40 / 60$ & 92,400 & 15,400 and 77,000 \\
$42 / 58$ & 387,200 & 17,600 and 369,600 \\
$44 / 56$ & 347,600 & 1,100 and 346,500 \\
$48 / 52$ & 600,600 & 231,000 and 369,600 \\
50 & $1,311,552$ & $352,123,200,264,000$ and 924,000 \\
\hline
\end{tabular}

In particular, $G$ has precisely 30 orbits in $H_{22}^{\prime}$. Complementary vectors of weight 50 are in the same $G$-orbit. 
Proof. Let $a_{i}=w_{i}\left(H_{22}^{\prime}\right)$ and $b_{i}=w_{i}\left(H_{78}^{\prime}\right)$. We recall that $H_{78}^{\prime}=\left(H_{22}^{\prime}\right)^{\perp}$. Hence the families $\left(a_{i}\right)$ and $\left(b_{i}\right)$ are related to each other by the MacWilliams identities.

We have $H_{22}^{\prime} \leqslant H_{78}^{\prime} \leqslant H_{99}$, as follows from (2.7); hence for every odd $i$ we have $a_{i}=$ $b_{i}=0$. Since $\mathbb{1} \in H_{22}^{\prime}$ we have $a_{i}=a_{100-i}$ for all $i$. Moreover, $\left\{x \mid x \in H_{22}^{\prime}\right.$ and $w(x) \equiv 0$ $(\bmod 4)\}=H_{21}$. In view of $(3.19)$ we therefore have the following information:

$$
\begin{aligned}
a_{i} & =0 \text { for } 0<i<32 \text { and } 78<i<100, \\
a_{0}=a_{100} & =1 \text { and from }(3.5) \text { every } a_{i} \text { with } i \equiv 0 \quad(\bmod 4) \text { is known. }
\end{aligned}
$$

So the only unknown weight numbers of $H_{22}^{\prime}$ with $i \leqslant 50$ are

$$
a_{34}, a_{38}, a_{42}, a_{46} \text { and } a_{50}
$$

On the other hand we know from (3.3) and (3.18) the values

$$
b_{0}=1, b_{2}=b_{4}=b_{6}=0 \text { and } b_{8}=17,600 .
$$

It follows from the general theory of MacWilliams identities that the weight distribution $\left(a_{i}\right)$ is uniquely determined by the known values of the $a_{i}$ and $b_{j}$, see e.g. [28]. Explicit calculations yield the values asserted in the theorem.

It remains to determine the $G$-orbits in $H_{22}^{\prime} \backslash H_{21}$. This will be done in a sequence of lemmas, as some detailed investigations are required. Note that the fact that all $G$-orbits in $W_{50}\left(H_{22}^{\prime}\right)$ have different length implies that complementary vectors of weight 50 are in the same $G$-orbit.

Recall from Section 2 that the permutation group $\bar{G} \cong \operatorname{Aut}(G)$ interchanges the codes $H_{22}^{\prime}$ and $H_{22}^{\prime \prime}$ and leaves $H_{21}$ invariant. Therefore we have a natural involutory correspondence between the $G$-orbits in $H_{22}^{\prime} \backslash H_{21}$ and those in $H_{22}^{\prime \prime} \backslash H_{21}$. We agree that $X^{\prime}$ and $X^{\prime \prime}$ will always denote $G$-orbits corresponding in this sense.

We start by considering the Higman vectors $x(m)=\alpha+m+B_{1}(m)$ which we know to belong to $H_{23} \backslash H_{22}$ from (2.7). Recall that we denote the $G_{\alpha}$-orbits $\mathcal{M}^{\prime}$ and $\mathcal{M}^{\prime \prime}$ on the heptads of $\mathcal{W}_{22}$ in such a way that $x(m) \in H_{22}^{\prime}$ if and only if $m \in \mathcal{M}^{\prime}$.

(3.21) Proposition.

Let $X_{0}^{\prime}=\left\{x(m) \mid m \in \mathcal{M}^{\prime}\right\} \cup\left\{x(m)+\mathbb{1} \mid m \in \mathcal{M}^{\prime}\right\}$. Then the following hold.

(1) $X_{0}^{\prime}$ is a $G$-orbit in $W_{50}\left(H_{22}^{\prime}\right) \backslash H_{21}$.

(2) $G_{x(m)} \cong \operatorname{PSU}\left(3,5^{2}\right)=\mathrm{U}_{3}(5)$ acts as a rank 3 group on the supports of $x(m)$ and $x(m)+\mathbb{1}$; the supports of $x(m)$ and $x(m)+\mathbb{1}$ are nonisomorphic $G_{x(m)}$-spaces. $G_{\{x(m), x(m)+\mathbb{1}\}} \cong \mathrm{P} \Sigma \mathrm{U}\left(3,5^{2}\right)$ acts transitively on $\Omega$.

(3) $\operatorname{supp}(x(m))=\Lambda_{7}(x(m))$ and $\operatorname{supp}(x(m)+\mathbb{1})=\Lambda_{15}(x(m))$.

(4) The mapping $m \mapsto\{x(m), x(m)+\mathbb{1}\}$ of $\mathcal{M}^{\prime}$ onto $X_{0}^{\prime} / H_{1}$ is bijective and a $G_{\alpha^{-}}$ morphism. In particular $\left|X_{0}^{\prime}\right|=2 \cdot 176=352$. 
Proof. It follows from (2.7) and (1.9) that $X_{0}^{\prime} / H_{1}=\left\{\{x, x+\mathbb{1}\} \mid x \in W_{50}\left(H_{22}^{\prime}\right)\right.$ and the Higman-Sims graph induces on $\operatorname{supp}(x)$ and on $\operatorname{supp}(x+\mathbb{1})$ a strongly regular graph of valency 7$\}$. Therefore $X_{0}^{\prime} / H_{1}$ and $X_{0}^{\prime}$ are invariant under $G$. It is immediate that $m \mapsto\{x(m), x(m)+\mathbb{1}\}$ is a bijective morphism of $G_{\alpha}$-spaces. It follows that $\left|X_{0}^{\prime} / H_{1}\right|=176$ and that $G$ acts transitively on $X_{0}^{\prime}$. From (1.3) and (1.9) we infer that $G_{x(m), \beta} \cong A_{7}$ for all $\beta \in \Omega$ and that $G_{x(m), \beta}$ acts as a rank 3 group on the supports of $x(m)$ and $x(m)+\mathbb{1}$, the supports being nonisomorphic $G_{x(m)}$-spaces. From D.G. Higman's result [14, 6.1], we may conclude that $G_{\{x(m), x(m)+\mathbb{1}\}} \cong \mathrm{P} \Sigma \mathrm{U}\left(3,5^{2}\right)$. The rest of the assertion easily follows.

Remark. Note that $G_{\{x(m), x(m)+1\}} \cong \mathrm{P} \Sigma \mathrm{U}\left(3,5^{2}\right)$ does not leave invariant the strongly regular graphs induced on $x(m)$ and $x(m)+\mathbb{1}$, but interchanges them blockwise. It follows from (2.7) that $\bar{G}_{\{x(m), x(m)+\mathbb{1}\}}=G_{\{x(m), x(m)+\mathbb{1}\}}$ where $\bar{G} \cong \operatorname{Aut}(G)$ is the automorphism group of the Higman-Sims graph. Hence even $\bar{G}$ induces on the supports of $x(m)$ and of $x(m)+\mathbb{1}$ only the group $\operatorname{PSU}\left(3,5^{2}\right)$, and not the complete automorphism group $\cong$ $\mathrm{P} \Sigma \mathrm{U}\left(3,5^{2}\right)$ of the strongly regular graph of valency 7 on 50 vertices.

By the convention above there is a $G$-orbit $X_{0}^{\prime \prime}$ in $W_{50}\left(H_{22}^{\prime \prime} \backslash H_{21}\right)$ which shares analogous properties. $X_{0}^{\prime}$ and $X_{0}^{\prime \prime}$ are interchanged by $\bar{G} \cong \operatorname{Aut}(G)$.

It is more complicated to deal with the remaining $G$-orbits. We use the connections in the Higman-Sims graph as a guide.

\section{(3.22) Lemma.}

Let $x=x(m) \in X_{0}^{\prime} ;$ then $\alpha \in \Lambda_{15}(x+\mathbb{1})$. We have $y=(x+\mathbb{1})+\Delta(\alpha) \in W_{42}\left(H_{22}^{\prime \prime}\right)$ and $y$ has the following properties.

(1) $G_{y}=G_{x, \alpha} \cong A_{7}$.

(2) $G_{y}$ has the following orbits in $\Omega$ :

$$
\begin{array}{lllll}
\Phi_{0}= & \{\alpha\} & = & \operatorname{supp}(x) \cap \Lambda_{7}(y), & \left|\Phi_{0}\right|=1 \\
\Phi_{1}= & \operatorname{supp}(m) & = & \operatorname{supp}(x) \cap \Lambda_{15}(y), & \left|\Phi_{1}\right|=7 \\
\Phi_{2}= & B_{1}(m) & = & \operatorname{supp}(x) \cap \Lambda_{11}(y), & \left|\Phi_{2}\right|=42 \\
\Phi_{3}=\Delta(\alpha)+m= & = & \operatorname{supp}(y+\mathbb{1}) \cap \operatorname{supp}(x+\mathbb{1}) \subseteq \Lambda_{7}(y), & \left|\Phi_{3}\right|=15 \\
\Phi_{4}= & B_{3}(m) & = & \operatorname{supp}(y) \cap \Lambda_{7}(y), & \left|\Phi_{4}\right|=35
\end{array}
$$

(3) The matrix of the Higman-Sims graph with respect to $\left(\Phi_{i}\right)_{0 \leqslant i \leqslant 4}$ is

$$
\left[\begin{array}{ccccc}
0 & 7 & 0 & 15 & 0 \\
1 & 0 & 6 & 0 & 15 \\
0 & 1 & 6 & 5 & 10 \\
1 & 0 & 14 & 0 & 7 \\
0 & 3 & 12 & 3 & 4
\end{array}\right] .
$$

Proof. The assertion follows from (1.13) since $G_{y}$ must leave invariant $\operatorname{supp}(y)$ and all $\Lambda_{i}(y)$. 


\section{(3.23) Proposition.}

Let $x=x(m) \in X_{0}^{\prime}$ and $y=(x+\mathbb{1})+\Delta(\alpha)$. Denote by $Y_{0}^{\prime \prime}$ the G-orbit containing $y$. Then $\left|Y_{0}^{\prime \prime}\right|=17,600$.

Proof. It follows from (3.21) that $\left|Y_{0}^{\prime \prime}\right|=\left|G: G_{y}\right|=352 \cdot 50=17,600$.

Note that every vector in $Y_{0}^{\prime \prime}$ (and $Y_{0}^{\prime}$ ) shares the properties of $y$ described in (3.22). In particular $\Lambda_{15}(y)$ is a unique $G_{y}$-orbit of length 7 for any $y \in Y_{0}^{\prime \prime}$. If $y \in Y_{0}^{\prime \prime}$ and $\beta \in \Lambda_{15}(y)$ then $z=y+\Delta(\beta) \in W_{34}\left(H_{22}^{\prime}\right)$ and $G_{z}$ contains a subgroup isomorphic to $A_{6}$. We show that $G$ acts transitively on $W_{34}\left(H_{22}^{\prime}\right)$ by arguments independent of the preceding discussion.

\section{(3.24) Lemma.}

Let $z \in W_{34}\left(H_{22}^{\prime}\right)$ and $\lambda_{i}=\left|\Lambda_{i}(z)\right|$. Then the following hold.

(1) $\lambda_{11}=12$ and $\lambda_{7}=88$.

(2) $G_{z}$ does not fix any point in $\Omega$.

Proof. $\lambda_{15}=0$ holds since $H_{22}^{\prime}$ has minimum weight 32 . The canonical equations by counting edges

$$
\begin{array}{ccc}
34 \cdot 22 & =7 \lambda_{7}+11 \lambda_{11} \\
100 & =\lambda_{7}+\lambda_{11}
\end{array}
$$

yield (1).

Assume that $G_{z}$ fixes a point $\beta \in \Omega$. From the first part of Theorem (3.20) follows $5,600 \geqslant\left|G: G_{z}\right|=\left|G: G_{\beta}\right|\left|G_{\beta}: G_{z}\right|=100\left|G_{\beta}: G_{z}\right|$ and $\left|G_{\beta}: G_{z}\right| \leqslant 56$. Since $G_{\beta} \cong M_{22}$ either $G_{\beta}=G_{z}$ or $G_{z}=G_{\beta \gamma}$ for some point $\beta \neq \gamma$. By considering the action of $G_{\beta}$ and of $G_{\beta \gamma}$ on $\Omega$ we see that $\left|\Lambda_{11}(z)\right|=12$ is impossible, a contradiction against (1). Thus (2) holds.

\section{(3.25) Proposition.}

$G$ acts transitively on $W_{34}\left(H_{22}^{\prime}\right)=Z^{\prime}$. Let $z \in Z^{\prime}$. Then the following hold.

(1) $G_{z} \cong M_{11}$, the simple group of order 7,920 .

(2) $\Lambda_{11}(z) \subseteq \operatorname{supp}(z)$ and $G_{z}$ acts triply-transitively on $\Lambda_{11}(z)$. If $\beta \in \Lambda_{11}(z)$ then $G_{z, \beta} \cong \operatorname{PSL}(2,11)$. The orbits of $G_{z}$ in $\Omega$ are $\Phi_{0}=\Lambda_{11}(z), \Phi_{1}=\operatorname{supp}(z) \cap \Lambda_{7}(z)$ and $\Phi_{2}=\operatorname{supp}(z+\mathbb{1})$ of lengths 12,22 and 66 .

(3) The matrix of the Higman-Sims graph with respect to $\left(\Phi_{i}\right)_{0 \leqslant i \leqslant 2}$ is

$$
\left[\begin{array}{ccc}
0 & 11 & 11 \\
6 & 1 & 15 \\
2 & 5 & 15
\end{array}\right] .
$$


Proof. (i) From the first part of Theorem (3.20) we have $w_{34}\left(H_{22}^{\prime}\right)=5,600 \not \equiv 0$ $(\bmod 11)$. Therefore there exists $z \in W_{34}\left(H_{22}^{\prime}\right)$ such that 11 divides $\left|G_{z}\right|$. From (3.24) we infer that any element of $G_{z}$ of order 11 fixes exactly one point in $\Lambda_{11}(z)$ and acts fixed-point-freely on $\Lambda_{7}(z)$. Since $G_{z}$ does not fix any point in $\Omega$ by (3.24) it follows that $G_{z}$ acts doubly-transitively on $\Lambda_{11}(z)$.

(ii) Let $\beta \in \Lambda_{11}(z)$. Then $\left|G_{z}: G_{z, \beta}\right|=12$ and we obtain that $\left|G_{z, \beta}\right|=|G| /(12 \mid G$ : $\left.G_{z} \mid\right) \geqslant 44,352,000 /(12 \cdot 5,600)=660$. On the other hand it follows from (1.3) that $G_{z, \beta}$ is isomorphic to a subgroup of $\operatorname{PSL}(2,11)$ whose order is 660 . Hence $G_{z, \beta} \cong \operatorname{PSL}(2,11)$. Consequently $G_{z}$ acts faithfully and triply-transitively on $\Lambda_{11}(z)$ and $G_{z} \cong M_{11}$ by [31]. Another consequence is $\left|G: G_{z}\right|=5,600$ which implies that $Z^{\prime}=W_{34}\left(H_{22}^{\prime}\right)$ is an orbit of $G$.

(iii) Since any element of $G_{z}$ of order 11 fixes exactly one point in $\Omega$ it follows that $\Lambda_{11}(z) \subseteq \operatorname{supp}(z)$. From the properties of the Higman-Sims graph we infer that $G_{z}$ acts transitively on $\operatorname{supp}(z) \cap \Lambda_{7}(z)$. Without loss we may assume that $\alpha \in \Lambda_{11}(z)$. Using the notation of (1.12) we then may conclude that for the endecad $e=\Delta(\alpha) z$ we have $B_{3}(e) \subseteq \operatorname{supp}(z+\mathbb{1})$. Since $G_{z, \alpha}$ acts transitively on $B_{3}(e)$, it now easily follows that $G_{z}$ acts transitively on $\operatorname{supp}(z+\mathbb{1})$ of cardinality 66 . The graph matrix in the assertion is now obtained simply by counting.

Remark. A vector $z \in Z^{\prime}$ may be constructed explicitly as follows: Let $e$ be an endecad such that $e$ and any heptad $m \in \mathcal{M}^{\prime}$ generate the same $M_{22}$-invariant subcode of the (shortened) Golay code of length 22. Then $z=\alpha+e+B_{1}(e)+B_{5}(e) \in Z^{\prime}$ and $z+\mathbb{1}=(e+\Delta(\alpha))+B_{3}(e)$, see $(1.12)$.

It is now quite obvious how the $G$-orbits $Z^{\prime}$ and $Y_{0}^{\prime \prime}$ are linked by the Higman-Sims graph:

$$
\begin{aligned}
Y_{0}^{\prime \prime} & =\left\{z+\Delta(\beta) \mid z \in Z^{\prime} \text { and } \beta \in \operatorname{supp}(z) \cap \Lambda_{7}(z)\right\} \text { and } \\
Z^{\prime} & =\left\{y+\Delta(\gamma) \mid y \in Y_{0}^{\prime \prime} \text { and } \gamma \in \Lambda_{15}(y)\right\} .
\end{aligned}
$$

It is clear that we may construct a second orbit $Y_{1}^{\prime \prime}$ in $W_{42}\left(H_{22}^{\prime \prime}\right)$ starting from $Z^{\prime}$ by making use of the $G_{z}$-orbit $\operatorname{supp}(z+\mathbb{1})$ instead of $\operatorname{supp}(z) \cap \Lambda_{7}(z)$.

\section{(3.26) Proposition.}

Let $z \in Z^{\prime}=W_{34}\left(H_{22}^{\prime}\right)$ and let $\gamma \in \operatorname{supp}(z+\mathbb{1})$. Then $y=z+\Delta(\gamma) \in W_{42}\left(H_{22}^{\prime \prime}\right)$. Denote by $Y_{1}^{\prime \prime}$ the G-orbit containing $y$. The following assertions hold.

(1) $G_{y}=G_{z, \gamma} \cong \Sigma_{5}$.

(2) $\left|Y_{1}^{\prime \prime}\right|=369,600$. 
(3) $G_{y}$ has exactly 9 orbits $\left(\Psi_{i}\right)_{0 \leqslant i \leqslant 8}$ in $\Omega$ which are defined as follows:

$$
\begin{array}{rlrl}
\Psi_{0} & =\Lambda_{11}(z) \cap \Delta(\gamma) ; & & \left|\Psi_{0}\right|=2 \text { and } \Psi_{0} \subseteq \Lambda_{11}(y) . \\
\Psi_{1} & =\Lambda_{11}(z) \backslash \Psi_{0} ; & & \left|\Psi_{1}\right|=10 \text { and } \Psi_{1} \subseteq \Lambda_{11}(y) . \\
\Psi_{2} & =\Lambda_{7}(z) \cap \operatorname{supp}(z) \cap \Delta(\gamma) ; & & \left|\Psi_{2}\right|=5 \text { and } \Psi_{2} \subseteq \Lambda_{7}(y) . \\
\Psi_{3}, \Psi_{4} & \subseteq \Lambda_{7}(z) \cap \operatorname{supp}(z) \backslash \Delta(\gamma) \text { such that } & & \left|\Psi_{3}\right|=5 \text { and }\left|\Psi_{4}\right|=12 ; \\
& & \Psi_{3} \subseteq \Lambda_{7}(y) \text { and } \Psi_{4} \subseteq \Lambda_{11}(y) \\
\Psi_{5} & =\{\gamma\}=\Lambda_{15}(y) . & & \\
\Psi_{6} & =\operatorname{supp}(z+\mathbb{1}) \cap \Delta(\gamma) ; & & \left|\Psi_{6}\right|=15 \text { and } \Psi_{6} \subseteq \Lambda_{7}(y) . \\
\Psi_{7}, \Psi_{8} & \subseteq \operatorname{supp}(z+\mathbb{1}) \backslash \Delta(\gamma) \text { such that } & & \left|\Psi_{7}\right|=20 \text { and }\left|\Psi_{8}\right|=30 \\
& & \Psi_{7} \subseteq \Lambda_{7}(y) \text { and } \Psi_{8} \subseteq \Lambda_{11}(y) .
\end{array}
$$

(4) $\operatorname{supp}(y)=\Psi_{1} \cup \Psi_{3} \cup \Psi_{4} \cup \Psi_{6}$ and $\Lambda_{7}(y)=\Psi_{2} \cup \Psi_{3} \cup \Psi_{6} \cup \Psi_{7}$.

(5) The matrix of the Higman-Sims graph with respect to $\left(\Psi_{i}\right)_{0 \leqslant i \leqslant 8}$ is

$$
\left[\begin{array}{ccccccccc}
0 & 0 & 0 & 5 & 6 & 1 & 0 & 10 & 0 \\
0 & 0 & 3 & 2 & 6 & 0 & 3 & 2 & 6 \\
0 & 6 & 0 & 1 & 0 & 1 & 0 & 8 & 6 \\
2 & 4 & 1 & 0 & 0 & 0 & 3 & 0 & 12 \\
1 & 5 & 0 & 0 & 1 & 0 & 5 & 5 & 5 \\
2 & 0 & 5 & 0 & 0 & 0 & 15 & 0 & 0 \\
0 & 2 & 0 & 1 & 4 & 1 & 0 & 4 & 10 \\
1 & 1 & 2 & 0 & 3 & 0 & 3 & 3 & 9 \\
0 & 2 & 1 & 2 & 2 & 0 & 5 & 6 & 4
\end{array}\right]
$$

Proof. It follows from (3.25) and [7, Table 3], that $G_{z, \gamma} \cong \Sigma_{5}$. Using the character table of $M_{11}$ we obtain that $G_{z, \gamma}$ has the nine orbits $\left(\Psi_{i}\right)_{0 \leqslant i \leqslant 8}$ in $\Omega$ as described in assertion (3). From (3.25) we derive the graph matrix given in (5) and it follows that $\Lambda_{15}(y)=\{\gamma\}=\Psi_{5}$. Therefore $G_{y}=G_{z, \gamma}$ and the rest of the proposition easily follows.

Since $w_{42}\left(H_{22}^{\prime \prime}\right)=17,600+369,600$ by the first part of Theorem (3.20) only the remaining $G$-orbits in $W_{50}\left(H_{22}^{\prime}\right)$ have to be determined. We use for the construction of these orbits the same ideas as for the construction of $Y_{1}^{\prime \prime}$. At first we prove a general lemma.

\section{(3.27) Lemma.}

Let $x \in W_{50}\left(H_{22}^{\prime}\right)$ and $\lambda_{i}=\left|\Lambda_{i}(x)\right|$. Then $\lambda_{7}=\lambda_{15}$.

Proof. Counting the edges of the Higman-Sims graph between $\operatorname{supp}(x)$ and $\Omega$ yields the equations

$$
\begin{aligned}
1,100=50 \cdot 22 & =7 \lambda_{7}+11 \lambda_{11}+15 \lambda_{15} \\
100 & =\lambda_{7}+\lambda_{11}+\lambda_{15}
\end{aligned}
$$

It follows $\lambda_{7}=\lambda_{15}$. 
Next we consider the incidence structure

$$
\mathcal{I}=\left(W_{42}\left(H_{22}^{\prime \prime}\right), W_{50}\left(H_{22}^{\prime}\right), \mathrm{I}\right)
$$

where $\mathrm{I}=\left\{(y, x) \mid y \in W_{42}\left(H_{22}^{\prime \prime}\right)\right.$ and there exists a $\beta \in \Omega$ such that $\left.x+y=\Delta(\beta)\right\}$. Note that $(x, y) \in \mathrm{I}$ implies that $x+y=\Delta(\beta)$ where $\beta \in \Lambda_{7}(y) \cap \Lambda_{15}(x)$.

It is obvious from the definition of $\mathcal{I}$ that $G$ acts on $\mathcal{I}$ as a group of automorphisms. We know that $G$ has exactly 2 orbits $Y_{0}^{\prime \prime}$ and $Y_{1}^{\prime \prime}$ in $W_{42}\left(H_{22}^{\prime \prime}\right)$ of lengths 17,600 and 369,600 respectively. It is our goal to determine the $G$-orbits in $W_{50}\left(H_{22}^{\prime}\right)$ via the $G$-orbits in I.

\section{(3.28) Proposition.}

$G$ has precisely 7 orbits in I:

$$
\begin{aligned}
& \mathrm{I}_{00}=\left\{(y, y+\Delta(\beta)) \mid y \in Y_{0}^{\prime \prime} \text { and } \beta \in \Phi_{0}(y)\right\}, \\
& \mathrm{I}_{01}=\left\{(y, y+\Delta(\beta)) \mid y \in Y_{0}^{\prime \prime} \text { and } \beta \in \Phi_{3}(y)\right\}, \\
& \mathrm{I}_{02}=\left\{(y, y+\Delta(\beta)) \mid y \in Y_{0}^{\prime \prime} \text { and } \beta \in \Phi_{4}(y)\right\}, \\
& \mathrm{I}_{10}=\left\{(y, y+\Delta(\beta)) \mid y \in Y_{1}^{\prime \prime} \text { and } \beta \in \Psi_{2}(y)\right\}, \\
& \mathrm{I}_{11}=\left\{(y, y+\Delta(\beta)) \mid y \in Y_{1}^{\prime \prime} \text { and } \beta \in \Psi_{3}(y)\right\}, \\
& \mathrm{I}_{12}=\left\{(y, y+\Delta(\beta)) \mid y \in Y_{1}^{\prime \prime} \text { and } \beta \in \Psi_{6}(y)\right\}, \\
& \mathrm{I}_{13}=\left\{(y, y+\Delta(\beta)) \mid y \in Y_{1}^{\prime \prime} \text { and } \beta \in \Psi_{7}(y)\right\} .
\end{aligned}
$$

(Here we write $\Phi_{i}(y)=\Phi_{i}$ in the sense of (3.22) and $\Psi_{i}(y)=\Psi_{i}$ in the sense of (3.26) for the sake of clarity.)

The orbit lengths are

$$
\begin{aligned}
& \left|\mathrm{I}_{00}\right|=17,600 \cdot 1=17,600, \\
& \left|\mathrm{I}_{01}\right|=17,600 \cdot 15=264,000, \\
& \left|\mathrm{I}_{02}\right|=17,600 \cdot 35=616,000, \\
& \left|\mathrm{I}_{10}\right|=369,600 \cdot 5=1,848,000, \\
& \left|\mathrm{I}_{11}\right|=369,600 \cdot 5=1,848,000, \\
& \left|\mathrm{I}_{12}\right|=369,600 \cdot 15=5,544,000, \\
& \left|\mathrm{I}_{13}\right|=369,600 \cdot 20=7,392,000 .
\end{aligned}
$$

Proof. The assertion is a straightforward consequence of (3.22) and (3.26). Note that for $y \in W_{42}\left(H_{22}^{\prime \prime}\right)$ we have $y+\Delta(\beta) \in W_{50}\left(H_{22}^{\prime}\right)$ if and only if $\beta \in \Lambda_{7}(y)$.

An immediate consequence of (3.28) is that there are at most $7 G$-orbits in $W_{50}\left(H_{22}^{\prime}\right)$. We know one of these $G$-orbits, $X_{0}^{\prime}$, from (3.21). $X_{0}^{\prime}$ corresponds uniquely to I00, as follows from (3.21). From the first part of Theorem (3.20) we know that $w_{50}\left(H_{22}^{\prime}\right)=1,311,552$. We construct now the remaining $G$-orbits by considering some particular vectors.

\section{(3.29) Proposition.}

Let $y=(x(m)+\mathbb{1})+\Delta(\alpha) \in Y_{0}^{\prime \prime}$ as defined in (3.22), let $\beta \in \Phi_{3}(y)=\Phi_{3}$ and let $x=y+\Delta(\beta)$. Set $X_{1}^{\prime}=\{x g \mid g \in G\}$. Then the following hold.

$$
\text { (1) } G_{x}=G_{y, \beta} \cong \operatorname{PSL}(2,7) \text {. }
$$


(2) $\left|X_{1}^{\prime}\right|=264,000$.

(3) $G_{x}$ has precisely 8 orbits $\left(\Theta_{i}\right)_{0 \leqslant i \leqslant 7}$ in $\Omega$.

$$
\begin{aligned}
& \Theta_{0}=\{\alpha\}, \quad\left|\Theta_{0}\right|=1 \text { and } \Theta_{0} \subseteq \Lambda_{7}(x) ; \\
& \Theta_{1}=\operatorname{supp}(m), \quad\left|\Theta_{1}\right|=7 \text { and } \Theta_{1} \subseteq \Lambda_{15}(x) \text {; } \\
& \Theta_{2}=\operatorname{supp}(y) \cap \Delta(\beta) \backslash\{\alpha\}, \quad\left|\Theta_{2}\right|=14 \quad \text { and } \quad \Theta_{2} \subseteq \Lambda_{11}(x) \text {; } \\
& \Theta_{3}=B_{1}(m) \backslash \Delta(\beta), \quad\left|\Theta_{3}\right|=28 \text { and } \Theta_{3} \subseteq \Lambda_{11}(x) \text {; } \\
& \Theta_{4}=\{\beta\}, \quad\left|\Theta_{4}\right|=1 \text { and } \Theta_{4} \subseteq \Lambda_{15}(x) \text {; } \\
& \Theta_{5}=\Delta(\alpha) \backslash \operatorname{supp}(m) \backslash\{\beta\}, \quad\left|\Theta_{5}\right|=14 \text { and } \Theta_{5} \subseteq \Lambda_{11}(x) \text {; } \\
& \Theta_{6} \quad=\quad \Delta(\beta) \cap \operatorname{supp}(y), \quad\left|\Theta_{6}\right|=7 \quad \text { and } \quad \Theta_{6} \subseteq \Lambda_{7}(x) \text {; } \\
& \Theta_{7}=B_{3}(m) \backslash \Delta(\beta), \quad\left|\Theta_{7}\right|=28 \text { and } \Theta_{7} \subseteq \Lambda_{11}(x) \text {. } \\
& \operatorname{supp}(x)=\Theta_{0} \cup \Theta_{1} \cup \Theta_{2} \cup \Theta_{7} \text {. }
\end{aligned}
$$

(4) The matrix of the Higman-Sims graph with respect to $\left(\Theta_{i}\right)_{0 \leqslant i \leqslant 7}$ is

$$
\left[\begin{array}{cccccccc}
0 & 7 & 0 & 0 & 1 & 14 & 0 & 0 \\
1 & 0 & 2 & 4 & 0 & 0 & 3 & 12 \\
0 & 1 & 0 & 6 & 1 & 4 & 0 & 10 \\
0 & 1 & 3 & 3 & 0 & 5 & 3 & 7 \\
1 & 0 & 14 & 0 & 0 & 0 & 7 & 0 \\
1 & 0 & 4 & 10 & 0 & 0 & 1 & 6 \\
0 & 3 & 0 & 12 & 1 & 2 & 0 & 4 \\
0 & 3 & 5 & 7 & 0 & 3 & 1 & 3
\end{array}\right]
$$

Proof. We make use of the results of (3.22): $G_{y} \cong A_{7}$ and $\left|G_{y}: G_{y, \beta}\right|=15$, hence $G_{y, \beta} \cong \operatorname{PSL}(2,7)$. Using the information given in (3.22) we easily obtain that $G_{y, \beta}$ has the orbits $\left(\Theta_{i}\right)_{0 \leqslant i \leqslant 7}$ in $\Omega$. Counting the edges of the Higman-Sims graph yields the graph matrix in (4) and shows that every $\Theta_{i}$ is left invariant by $G_{x}$. It follows $G_{x}=G_{y, \beta}$, and hence $\left|X_{1}^{\prime}\right|=264,000$. The rest of the assertion is now obvious.

Remark. It can be deduced from (3.21) and (3.22) that $x$ and $x+\mathbb{1}$ are in the same $G$-orbit $X_{1}^{\prime}$. This will follow also by simple numerical reasons when the proof of Theorem (3.20) is complete. It should be noted that the Higman-Sims graph induces on $\Theta_{1} \cup \Theta_{6}$ the incidence graph of the projective plane of order 2, displaying in this way the well known isomorphy $\operatorname{PSL}(2,7) \cong \operatorname{PSL}(3,2)$ and $\operatorname{PGL}(2,7)$ acting as correlation group of the projective plane of order 2 .

\section{(3.30) Lemma.}

Let $y=(x(m)+\mathbb{1})+\Delta(\alpha) \in Y_{0}^{\prime \prime}$ as defined in (3.22), let $\gamma \in \Phi_{4}(y)=\Phi_{4}$ and let $x=y+\Delta(\gamma)$. Set $X_{2}^{\prime}=\{x g \mid g \in G\}$. Let $a=\left|G_{x}: G_{y, \gamma}\right|$. Then the following hold.

$$
\begin{aligned}
& \text { (1) } G_{y, \gamma} \cong \Sigma_{4}{ }^{2} \wedge \Sigma_{3} . \\
& \text { (2) }\left|X_{2}^{\prime}\right|=616,000 / a
\end{aligned}
$$


(3) $G_{y, \gamma}$ has precisely 12 orbits $\left(\Xi_{i}\right)_{0 \leqslant i \leqslant 11}$ in $\Omega$ :

$\Xi_{0}=\{\alpha\}, \quad\left|\Xi_{0}\right|=1$ and $\Xi_{0} \subseteq \Lambda_{7}(x)$;

$\Xi_{1}$ and $\Xi_{2}$ are the orbits of $G_{y, \gamma}$ in $m$ such that $\left|\Xi_{1}\right|=4$ and $\left|\Xi_{2}\right|=3$;

$\Xi_{1} \cup \Xi_{2} \subseteq \Lambda_{15}(x)$;

$\Xi_{3}=\Delta(\gamma) \cap B_{1}(m),\left|\Xi_{3}\right|=12$ and $\Xi_{3} \subseteq \Lambda_{11}(x)$;

$\Xi_{4}$ and $\Xi_{5}$ are the orbits of $G_{y, \gamma}$ in $B_{1}(m) \backslash \Delta(\gamma)$ such that $\left|\Xi_{4}\right|=12$ and $\left|\Xi_{5}\right|=18$;

$\Xi_{6}$ and $\Xi_{7}$ are the orbits of $G_{y, \gamma}$ in $\Delta(\alpha) \backslash m$ such that $\left|\Xi_{6}\right|=12$ and $\left|\Xi_{7}\right|=3$;

$\Xi_{8}=\{\gamma\},\left|\Xi_{8}\right|=1$ and $\Xi_{8} \subseteq \Lambda_{15}(x)$;

$\Xi_{9}=\Delta(\gamma) \cap B_{3}(m),\left|\Xi_{9}\right|=4$ and $\Xi_{9} \subseteq \Lambda_{7}(x)$;

$\Xi_{10}$ and $\Xi_{11}$ are the orbits of $G_{y, \gamma}$ in $B_{3}(m) \backslash(\gamma \cup \Delta(\gamma))$ such that

$$
\left|\Xi_{10}\right|=12 \text { and }\left|\Xi_{11}\right|=18 \text {; }
$$

$\operatorname{supp}(x)=\Xi_{1} \cup \Xi_{3} \cup \Xi_{7} \cup \Xi_{8} \cup \Xi_{10} \cup \Xi_{11}$.

(4) The matrix of the Higman-Sims graph with respect to $\left(\Xi_{i}\right)_{0 \leqslant i \leqslant 11}$ is

$$
\left[\begin{array}{cccccccccccc}
0 & 4 & 3 & 0 & 0 & 0 & 12 & 3 & 0 & 0 & 0 & 0 \\
1 & 0 & 0 & 3 & 3 & 0 & 0 & 0 & 0 & 3 & 3 & 9 \\
1 & 0 & 0 & 0 & 0 & 6 & 0 & 0 & 1 & 0 & 8 & 6 \\
0 & 1 & 0 & 0 & 3 & 3 & 5 & 0 & 1 & 0 & 3 & 6 \\
0 & 1 & 0 & 3 & 0 & 3 & 3 & 2 & 0 & 1 & 3 & 6 \\
0 & 0 & 1 & 2 & 2 & 2 & 4 & 1 & 0 & 2 & 4 & 4 \\
1 & 0 & 0 & 5 & 3 & 6 & 0 & 0 & 0 & 1 & 3 & 3 \\
1 & 0 & 0 & 0 & 8 & 6 & 0 & 0 & 1 & 0 & 0 & 6 \\
0 & 0 & 3 & 12 & 0 & 0 & 0 & 3 & 0 & 4 & 0 & 0 \\
0 & 3 & 0 & 0 & 3 & 9 & 3 & 0 & 1 & 0 & 3 & 0 \\
0 & 1 & 2 & 3 & 3 & 6 & 3 & 0 & 0 & 1 & 0 & 3 \\
0 & 2 & 1 & 4 & 4 & 4 & 2 & 1 & 0 & 0 & 2 & 2
\end{array}\right] .
$$

(5) $\Lambda_{15}(x) \cap \operatorname{supp}(x)=\Xi_{1} \cup \Xi_{8}$ has 5 points. $\Lambda_{15}(x) \cap \operatorname{supp}(x+\mathbb{1})=\Xi_{2} \cup \Xi_{4}$ has 15 points. $G_{x}$ has at least 2 orbits in $\Lambda_{15}(x)$.

(6) $\left|\Lambda_{7}(x)\right|=\left|\Lambda_{15}(x)\right|=20$ and $\left|\Lambda_{11}(x)\right|=60$.

Proof. (1) follows from (3.22) since $\gamma \in \Phi_{4}(y)$. (2) follows from (1). The orbits $\Xi_{i}$ and the graph matrix are obtained by studying the action of $G_{y, \gamma} \cong \Sigma_{4}{ }^{2} \lambda \Sigma_{3}$ using (3.22). The remaining part of the assertion follows by inspection.

\section{(3.31) Lemma.}

Let $y \in Y_{1}^{\prime \prime}$ as defined in (3.26) and let $\delta \in \Psi_{6}(y)=\Psi_{6}$. Let $b=\left|G_{x}: G_{y, \delta}\right|$. Set $x=y+\Delta(\delta)$ and $X_{3}^{\prime}=\{x g \mid g \in G\}$. Then the following hold.

$$
\text { (1) } G_{y, \delta} \cong D_{8}
$$


(2) $\left|X_{3}^{\prime}\right|=5,544,000 / b$.

(3) $G_{y, \delta}$ has precisely 26 orbits in $\Omega, 13$ in $\operatorname{supp}(x)$ and 13 in $\operatorname{supp}(x+\mathbb{1})$.

(4) $x+\mathbb{1} \in X_{3}^{\prime}$.

(5) $\left|\Lambda_{15}(x) \cap \operatorname{supp}(x)\right|=6$ and $\left|\Lambda_{15}(x) \cap \operatorname{supp}(x+\mathbb{1})\right|=8,\left|\Lambda_{7}(x)\right|=\left|\Lambda_{15}(x)\right|=$ 14 and $\left|\Lambda_{11}(x)\right|=72$. $G_{x}$ has at least 2 orbits in $\Lambda_{15}(x)$.

Proof. It follows from (3.26) that $G_{y, \delta} \cong D_{8}$; hence (1) holds and because of $G_{y, \delta} \leqslant G_{x}$ we have also (2).

From (3.26) we also infer that $G_{y, \delta}$ has 13 orbits (of lengths 1, 1, 2, 2, 4, 4, 4, 4, 4, 4, 4, 8, 8) in $\operatorname{supp}(x)$ and 13 orbits (with the same lengths) in $\operatorname{supp}(x+\mathbb{1})$. One easily checks that $\Lambda_{7}(x) \cap \operatorname{supp}(x) \neq \emptyset$ and $\Lambda_{7}(x) \cap \operatorname{supp}(x+\mathbb{1}) \neq \emptyset$. Therefore $G_{x}$ has at least 2 orbits in $\Lambda_{7}(x)$. It now follows from (3.28) that $G$ has exactly the orbits $X_{0}^{\prime}, X_{1}^{\prime}, X_{2}^{\prime}$ and $X_{3}^{\prime}$ in $W_{50}\left(H_{22}^{\prime}\right)$ and that every $x \in W_{50}\left(H_{22}^{\prime}\right)$ is in the same $G$-orbit as its complementary vector $x+\mathbb{1}$. One checks with the help of (3.26) that $\Lambda_{7}(x) \cap \operatorname{supp}(x)$ contains two $G_{y, \delta}$-orbits of length 4 each and that $\Lambda_{15}(x) \cap \operatorname{supp}(x)$ contains $3 G_{y, \delta}$-orbits of lengths $1,1,4$. All remaining $G_{y, \delta}$-orbits in $\operatorname{supp}(x)$ are in $\Lambda_{11}(x)$. Since $x+\mathbb{1} \in X_{3}^{\prime}$ we obtain (5).

Remark. For the proof of (3.31) it is not necessary to compute completely the matrix of the Higman-Sims Graph with respect to the orbits of $G_{y, \delta}$; indirect arguments are sufficient.

As a side result of the proof of (3.31) we note the following:

\section{(3.32) Proposition.}

The $G$-orbits in $W_{50}\left(H_{22}^{\prime}\right)$ are $X_{0}^{\prime}, X_{1}^{\prime}, X_{2}^{\prime}, X_{3}^{\prime}$. For all $x \in W_{50}\left(H_{22}^{\prime}\right)$ the complementary vectors $x$ and $x+\mathbb{1}$ are in the same $G$-orbit.

It remains to determine the lengths of the orbits $X_{2}^{\prime}$ and $X_{3}^{\prime}$ and the structure of the corresponding point stabilizers.

\section{(3.33) Proposition.}

$\left|X_{3}^{\prime}\right|=924,000$ and $G_{x} \cong \mathrm{GL}(2,3)$ for $x \in X_{3}^{\prime}$. The center $Z\left(G_{x}\right) \cong Z_{2}$ is spanned by an involution with fixed-points, thus $G_{x}$ is contained in the centralizer in $G=$ HS of a unique involution which has 10 fixed-points in $\operatorname{supp}(x)$ and 10 fixed-points in $\operatorname{supp}(x+\mathbb{1})$.

$G_{x}=G_{x+\mathbb{1}}$ has exactly 2 orbits in $\Lambda_{15}(x)$, namely $\Lambda_{15}(x) \cap \operatorname{supp}(x)$ of length 6 and $\Lambda_{15}(x) \cap \operatorname{supp}(x+\mathbb{1})$ of length 8 , correspondingly exactly 2 orbits in $\Lambda_{7}(x)$, namely $\Lambda_{7}(x) \cap$ $\operatorname{supp}(x)$ of length 8 and $\Lambda_{7}(x) \cap \operatorname{supp}(x+\mathbb{1})$ of length 6 . The orbit lengths of $G_{x}$ in $\Lambda_{11}(x) \cap \operatorname{supp}(x)$ resp. in $\Lambda_{11}(x) \cap \operatorname{supp}(x+\mathbb{1})$ are $4,8,24$.

Proof. Let $x \in X_{3}^{\prime}$ as defined in (3.31). It follows from (3.28) and the known properties of $Y_{i}^{\prime \prime}$ and $X_{j}^{\prime}$ that $G_{x}$ has the orbits $\Lambda_{15}(x) \cap \operatorname{supp}(x)$ of length 6 and $\Lambda_{15}(x) \cap \operatorname{supp}(x+\mathbb{1})$ of length 8 in $\Lambda_{15}(x)$. It follows that $b=\left|G_{x}: G_{y, \delta}\right|=6$, therefore $\left|X_{3}^{\prime}\right|=924,000$ and $\left|G_{x}\right|=48$. (Note that $\delta \in \Lambda_{15}(x) \cap \operatorname{supp}(x)$, hence $G_{y, \delta}=G_{x, \delta}$.)

An explicit computation of a vector $x \in X_{3}^{\prime}$ which may also be carried out with GAP [10] verifies the remaining assertions. Note that an involution in $G$ which is not fixed-point free has exactly 20 fixed-points by the character table of $G=$ HS, see the ATLAS [1]. 


\section{(3.34) Proposition.}

$\left|X_{2}^{\prime}\right|=123,200$ and $G_{x} \cong \Sigma_{5}{ }^{2} \lambda \Sigma_{3}$ for $x \in X_{2}^{\prime}$. $G_{x}$ has exactly 2 orbits in $\Lambda_{15}(x)$, namely $\Lambda_{15}(x) \cap \operatorname{supp}(x)$ of length 5 and $\Lambda_{15}(x) \cap \operatorname{supp}(x+\mathbb{1})$ of length 15.

Proof. We may assume that $x \in X_{2}^{\prime}$ is as defined in (3.30). The assertion is now easily obtained using (3.28), (3.30) and (3.32).

Remark. It is not hard to show that in (3.34) the stabilizer $G_{x}$ has the orbits

$$
\begin{gathered}
\Lambda_{15}(x) \cap \operatorname{supp}(x) \text { of length } 5, \\
\Lambda_{7}(x) \cap \operatorname{supp}(x) \text { of length } 15, \\
\Lambda_{11}(x) \cap \operatorname{supp}(x) \text { of length } 30, \\
\Lambda_{7}(x) \cap \operatorname{supp}(x+\mathbb{1}) \text { of length } 5, \\
\Lambda_{15}(x) \cap \operatorname{supp}(x+\mathbb{1}) \text { of length } 15 \text { and } \\
\Lambda_{11}(x) \cap \operatorname{supp}(x+\mathbb{1}) \text { of length } 30 .
\end{gathered}
$$

The matrix of the Higman-Sims graph with respect to these $G_{x}$-orbits (in the given order) is

$$
\left[\begin{array}{cccccc}
0 & 3 & 12 & 4 & 3 & 0 \\
1 & 0 & 6 & 1 & 8 & 6 \\
2 & 3 & 6 & 0 & 3 & 8 \\
4 & 3 & 0 & 0 & 3 & 12 \\
1 & 8 & 6 & 1 & 0 & 6 \\
0 & 3 & 8 & 2 & 3 & 6
\end{array}\right]
$$

One may check that this assertion agrees with Lemma (3.30).

The proof of Theorem (3.20) is now complete.

We conclude this discussion by a diagram (Figure 4) which displays the $G$-invariant relations between the orbits $Z^{\prime}, Y_{i}^{\prime \prime}$ and $X_{j}^{\prime}$ given by addition of adjacency vectors $\Delta(\xi), \xi \in$ $\Omega$. The strokes indicate the $G$-invariant relations; the numbers at the end of the strokes indicate the length of the stabilizer orbit belonging to the relation orbit of $G$. Note that $Y_{1}^{\prime \prime}$ is joined to $X_{2}^{\prime}$ via the stabilizer orbit $\Psi_{2}$, see (3.26) and (3.28).

\section{(3.35) Corollary.}

The weight distribution of $\mathrm{H}_{23}$ is as described in the following table:

\begin{tabular}{|c|c|c|c|c|c|c|c|}
\hline$i$ & $0 / 100$ & $22 / 78$ & $30 / 70$ & $32 / 68$ & $34 / 66$ & $36 / 64$ & $38 / 62$ \\
\hline$w_{i}\left(H_{23}\right)$ & 1 & 100 & 1,100 & 3,850 & 11,200 & 4,125 & 38,500 \\
\hline \hline$i$ & $40 / 60$ & $42 / 58$ & $44 / 56$ & $46 / 54$ & $48 / 52$ & 50 & \\
\hline$w_{i}\left(H_{23}\right)$ & 92,400 & 968,000 & 347,600 & 485,100 & 600,600 & $3,283,456$ & \\
\hline
\end{tabular}

Proof. The assertion follows from the known structure of $H_{23} / H_{21}$ together with (3.11) and (3.20). 


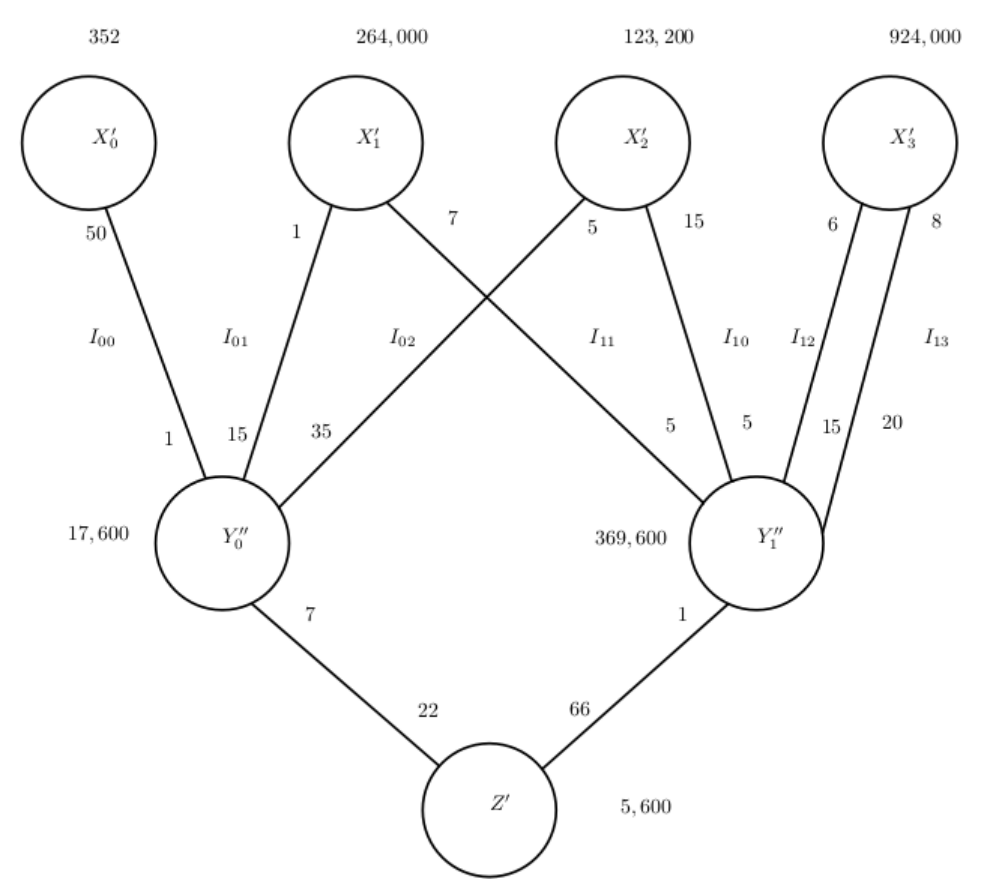

Figure 4: The $G$-invariant relations between $Z^{\prime}, Y_{i}^{\prime \prime}$ and $X_{j}^{\prime}$.

The weight enumerators of $H_{77}, H_{78}^{\prime}$ and $H_{78}^{\prime \prime}$ finally can be determined by MacWilliams transformation. (These computations have been carried out first in 1980 by F.H Florian at the Rechenzentrum of Tübingen University using the ALDES program for computing with large numbers; nowadays it is easy to obtain the result by suitable computer algebra software like e.g. GAP [10].)

\section{(3.36) Proposition.}

The weight distributions of $H_{77}, H_{78}^{\prime}$ and $H_{78}^{\prime \prime}$ are as given in Table 4 .

Proof. The assertion follows from (3.35) and (3.20) by the MacWilliams transformation.

Remark. From (2.7) it directly follows that there exist $G$-invariant linear forms $f^{\prime}$, $f^{\prime \prime}$ of $H_{79}$ such that $x f^{\prime} \neq 0 \neq x f^{\prime \prime}$ for all $x \in H_{78} \backslash H_{77}$ and all $x \in H_{22} \backslash H_{21}$. The results of Section 3 therefore imply that we may obtain by adding two "parity checks" a (binary) $(102,78)$-code of minimum weight 8 and a $(102,22)$-code of minimum weight 24 .

\section{A model of G. Higman's geometry}

In this section we consider the embedding of the Higman vectors $x(m)=\alpha+m+B_{1}(m)$ in the code $H_{23}$ (where $m$ is a heptad of $\mathcal{W}_{22}$ ) and derive in this way a natural model of G. Higman's geometry [17] on which the Higman-Sims group $G=$ HS acts as a group 


\begin{tabular}{|c|r|r|}
\hline$i$ & $w_{i}\left(H_{77}\right)$ & $w_{i}\left(H_{78}^{\prime}\right)=w_{i}\left(H_{78}^{\prime \prime}\right)$ \\
\hline $0 / 100$ & 1 & 1 \\
$8 / 92$ & 119,625 & 137,225 \\
$10 / 90$ & $3,351,040$ & $7,231,840$ \\
$12 / 88$ & $262,194,275$ & $511,741,475$ \\
$14 / 86$ & $10,460,595,200$ & $20,997,046,400$ \\
$16 / 84$ & $321,165,892,575$ & $642,104,180,575$ \\
$18 / 82$ & $7,309,692,544,000$ & $14,620,696,059,200$ \\
$20 / 80$ & $127,793,807,058,995$ & $255,580,729,090,995$ \\
$22 / 78$ & $1,748,088,230,732,800$ & $3,496,191,224,323,200$ \\
$24 / 76$ & $19,020,111,451,577,775$ & $38,040,223,036,760,175$ \\
$26 / 74$ & $166,791,619,843,340,800$ & $333,583,215,539,666,400$ \\
$28 / 72$ & $1,191,810,146,845,445,325$ & $2,383,620,193,904,285,325$ \\
$30 / 70$ & $7,002,911,342,735,052,800$ & $14,005,823,013,894,187,520$ \\
$32 / 68$ & $34,096,837,242,289,671,850$ & $68,193,674,589,734,420,650$ \\
$34 / 66$ & $138,453,825,199,499,980,800$ & $276,907,649,363,395,385,600$ \\
$36 / 64$ & $471,402,307,704,520,229,125$ & $942,804,613,467,381,809,925$ \\
$38 / 62$ & $1,351,845,015,778,272,153,600$ & $2,703,690,046,024,936,460,800$ \\
$40 / 60$ & $3,277,357,630,135,281,557,850$ & $6,554,715,226,694,274,576,090$ \\
$42 / 58$ & $6,737,425,031,963,982,617,600$ & $13,474,850,114,631,510,262,000$ \\
$44 / 66$ & $11,772,688,854,024,011,418,750$ & $23,545,377,636,355,278,743,550$ \\
$46 / 64$ & $17,516,850,935,961,851,443,200$ & $35,003,701,987,289,528,723,200$ \\
$48 / 52$ & $22,222,175,416,214,614,898,750$ & $44,444,350,658,167,367,673,150$ \\
50 & $24,054,370,909,850,203,084,800$ & $48,108,742,023,087,188,141,952$ \\
\hline
\end{tabular}

Table 4: The weight distributions of $H_{77}, H_{78}^{\prime}$ and $H_{78}^{\prime \prime}$.

of automorphisms. We thereby obtain an easy direct proof that G. Higman's simple group [17] is in fact isomorphic to the Higman-Sims group. Former proofs of this well known fact involve computer calculations [30], the use of the Leech lattice [7] or rather complicated combinatorial investigations, see [32, 33]; for another elementary proof of the isomorphy see [7]. The code theoretic construction of G. Higman's geometry also provides for a simple explanation of G. Higman's "natural correspondence" between the unordered pairs of points and quadrics, not induced by a bijection.

We shall consider all possible sums $x\left(m_{1}\right)+x\left(m_{2}\right)$ of Higman vectors. Recall that the $M_{22}$-orbits on the set of heptads of $\mathcal{W}_{22}$ are denoted by $\mathcal{M}^{\prime}$ and $\mathcal{M}^{\prime \prime}$ and that the notation for the codes is chosen so that $x(m) \in H_{22}^{\prime}$ if and only if $m \in \mathcal{M}^{\prime}$. The additive structure of $H_{23} / H_{21}$ leads to the following fact.

\section{(4.1) Lemma.}

Let $m_{1}, m_{2} \in \mathcal{M}^{\prime} \cup \mathcal{M}^{\prime \prime}$. Then the following hold.

(1) $x\left(m_{1}\right)+x\left(m_{2}\right) \in H_{21}$ if and only if $\left|\left\{m_{1}, m_{2}\right\} \cap \mathcal{M}^{\prime}\right|$ is even. 
(2) $x\left(m_{1}\right)+x\left(m_{2}\right) \in H_{22} \backslash H_{21}$ if and only if $\left|\left\{m_{1}, m_{2}\right\} \cap \mathcal{M}^{\prime}\right|=1$.

Proof. The assertions follow from (2.7).

More precise information is given by computing the weights:

(4.2) Lemma.

Let $m_{1}, m_{2} \in \mathcal{M}^{\prime} \cup \mathcal{M}^{\prime \prime}$ and let $d=w\left(m_{1} m_{2}\right)=\left|\operatorname{supp}\left(m_{1}\right) \cap \operatorname{supp}\left(m_{2}\right)\right|$. Then $w=$ $w\left(x\left(m_{1}\right)+x\left(m_{2}\right)\right)$ is given by the following table.

\begin{tabular}{|c|cccccc|}
\hline$d$ & 0 & 1 & 2 & 3 & 4 & 7 \\
\hline$w$ & 70 & 60 & 50 & 40 & 30 & 0 \\
\hline
\end{tabular}

Proof. The assertion easily follows from the definition of $x\left(m_{i}\right)$ by using the Leech triangle, [7, p.226]. (Observe that the heptads may be considered as shortened octads of the (extended) Golay code of length 24.)

The results in (4.2) become more symmetrical when we pass to the factor space $H_{23} / H_{1}$ of complementary vectors. For convenience of notation let $\widehat{x}=\{x, x+\mathbb{1}\} \in H_{23} / H_{1}$ for $x \in H_{23}$ and let $\widehat{x}(m)=\widehat{x(m)}$. As before in Section 3 the weight $w(\widehat{x})$ of $\widehat{x}$ is defined by $w(\widehat{x})=\{w(x), w(x+\mathbb{1})\}$.

\section{(4.3) Corollary.}

Let $m_{1}, m_{2} \in \mathcal{M}^{\prime} \cup \mathcal{M}^{\prime \prime}$. Then the weight $\widehat{w}=w\left(\widehat{x}\left(m_{1}\right)+\widehat{x}\left(m_{2}\right)\right)$ as a function of $d=w\left(m_{1} m_{2}\right)$ is given by the following table

\begin{tabular}{|c|ccc|ccc|}
\hline$d$ & 0 & 4 & 2 & 1 & 3 & 7 \\
\hline$\widehat{w}$ & $\{30,70\}$ & $\{30,70\}$ & $\{50,50\}$ & $\{40,60\}$ & $\{40,60\}$ & $\{0,100\}$ \\
\hline
\end{tabular}

Proof. The assertion is essentially a restatement of (4.2) using an ordering according to (4.1).

In (4.3) we see that the cases $d=0$ and $d=4$ (resp. $d=1$ and $d=3$ ) yield the same weights. In the following we shall use the $G$-orbits structure known from Section 3 to explain this observation.

From Section 3 we know that $G$ has exactly one orbit $X$ in $H_{23} / H_{1}$ of elements of weights $\{30,70\}$ of length $|X|=1,100$ and that $G$ has exactly 2 orbits in $H_{23} / H_{1}$ of elements of weight $\{40,60\}$, one of them - say $Y$ - of length 15,400 , the other of length 77,000 . Both orbits are in fact contained in $H_{22} / H_{1}$. Moreover, $G$ has exactly 2 orbits in $H_{22} / H_{1}$ of elements of weight $\{50,50\}$, one of them - say $Z$ - of length $|Z|=22,176$, the other of length 308,000 . All these orbits are also $\bar{G}$-invariant.

In addition we set $X^{\prime}=X_{0}^{\prime} / H_{1}$ and $X^{\prime \prime}=X_{0}^{\prime \prime} / H_{1}$ where $X_{0}$ and $X_{0}^{\prime \prime}$ are the $G$-orbits of Higman vectors such that $X_{0}^{\prime} \subseteq H_{22}^{\prime}$ and $X_{0}^{\prime \prime} \subseteq H_{22}^{\prime \prime}$, the modulo notation / $H_{1}$ having the obvious meaning. Then $\left|X^{\prime}\right|=\left|X^{\prime \prime}\right|=176$. Note that $G$ acts transitively on $X^{\prime}$ and $X^{\prime \prime}$ and that the stabilizer in $G$ of an element of $X^{\prime} \cup X^{\prime \prime}$ is isomorphic to $\operatorname{P\Sigma U}\left(3,5^{2}\right)$. $\bar{G} \cong \operatorname{Aut}(G)$ acts transitively on $X^{\prime} \cup X^{\prime \prime}$. 


\section{(4.4) Lemma.}

Let $x_{1}, x_{2} \in X^{\prime} \cup X^{\prime \prime}$. Then $x_{1}+x_{2} \in X \cup Y \cup Z \cup\{0\}$.

Proof. In view of Corollary (4.3) we only have to show that $x_{1}+x_{2}$ does not belong to the orbit of length 77,000 of elements of weight $\{40,60\}$ and not to the orbit of length 308,000 of elements of weight $\{50,50\}$. But this claim follows from (1.3) and (4.3), as $176(105+70)<77,000$ and $176 \cdot 126<308,000$.

As a consequence of (4.4) we may study the ternary relation $R=\left\{\left(x_{1}, x_{2}, x_{1}+x_{2}\right) \mid\right.$ $\left.x_{1}, x_{2} \in X^{\prime} \cup X^{\prime \prime}\right\} \subseteq\left(X^{\prime} \cup X^{\prime \prime}\right)^{2} \times(X \cup Y \cup Z \cup\{0\})$ in some detail. Of course, this relation is $\bar{G}$-invariant.

(4.5) Proposition. (1) $R^{\prime}=\left\{\left(x_{1}, x_{2}, x_{1}+x_{2}\right) \mid x_{1}, x_{2} \in X^{\prime}\right.$ and $\left.x_{1} \neq x_{2}\right\}$ is a G-orbit in $\left(X^{\prime}\right)^{2} \times Y$ of length $176 \cdot 175=30,800=2 \cdot 15,400$.

(2) $R^{\prime \prime}=\left\{\left(x_{1}, x_{2}, x_{1}+x_{2}\right) \mid x_{1}, x_{2} \in X^{\prime \prime}\right.$ and $\left.x_{1} \neq x_{2}\right\}$ is a G-orbit in $\left(X^{\prime \prime}\right)^{2} \times Y$ of length $176 \cdot 175=30,800=2 \cdot 15,400$.

(3) $X^{\prime}$ and $X^{\prime \prime}$ are interchanged by $\bar{G}$.

Proof. The assertion follows from (4.4), (3.11) and (3.20).

(4.6) Corollary.

$G$ acts doubly-transitively on $X^{\prime}$ and $X^{\prime \prime}$.

Proof. $G$ acts transitively on $Y$. From (4.5) it follows that $G$ is 2-homogeneous on $X^{\prime}$ and $X^{\prime \prime}$. Therefore, since $G$ is even, $G$ is doubly-transitive.

\section{(4.7) Corollary.}

There is a G-invariant natural correspondence $\Theta: X^{\prime\{2\}} \rightarrow X^{\prime \prime}\{2\}$ given by $\left\{x_{1}, x_{2}\right\} \Theta=$ $\left\{y_{1}, y_{2}\right\}$ where $y_{1}+y_{2}=x_{1}+x_{2}$.

Proof. The assertion follows also from (4.5).

Note that $\Theta$ is not induced by a bijection $X^{\prime} \rightarrow X^{\prime \prime}$ since $X^{\prime}$ and $X^{\prime \prime}$ are nonisomorphic $G$-sets.

Another particular $G$-orbit in $R$ can be used to construct a model of G. Higman's geometry.

(4.8) Proposition. (1) $R_{1}=\left\{\left(x_{1}, x_{2}, x\right) \mid x_{1} \in X^{\prime}, x_{2} \in X^{\prime \prime}, x \in X\right.$ and $\left.x=x_{1}+x_{2}\right\}$ is a $G$-orbit in $X^{\prime} \times X^{\prime \prime} \times X$ of length $176 \cdot 50=8,800=8 \cdot 1,100 . R_{2}=\left\{\left(x_{1}, x_{2}, x\right) \mid\right.$ $x_{1} \in X^{\prime \prime}, x_{2} \in X^{\prime}, x \in X$ and $\left.x=x_{1}+x_{2}\right\}$ is a $G$-orbit in $X^{\prime \prime} \times X^{\prime} \times X$ of length $176 \cdot 50=8,800=8 \cdot 1,100$.

(2) $R_{1}$ and $R_{2}$ are interchanged by $\bar{G}$.

Proof. The assertion follows from (4.4) and Section 3. 


\section{(4.9) Corollary.}

The stabilizer in $G$ of an element $x \in X$ is isomorphic to the symmetric group $\Sigma_{8}$.

Proof. $|X|=1,100$ implies $\left|G_{x}\right|=40,320=8 !=\left|\Sigma_{8}\right|$. The assertion now follows from (4.8).

We are now in a position to define a model of G. Higman's geometry: Call the elements of $X^{\prime}$ points, the elements of $X$ conics and the elements of $X^{\prime \prime}$ quadrics.

The $G$-invariant relation $R_{1}$ (or equivalently $R_{2}$ ) induces the following incidence structures by coordinate restriction:

$$
\begin{aligned}
& \mathcal{I}^{\prime}=\left(X^{\prime}, X, \mathrm{I}_{\text {ind }}\right) \quad \text { (point-conic structure) } \\
& \mathcal{I}^{\prime \prime}=\left(X^{\prime \prime}, X, \mathrm{I}_{\text {ind }}\right) \quad \text { (quadric-conic structure) } \\
& \mathcal{I}=\left(X^{\prime}, X^{\prime \prime}, \mathrm{I}_{\text {ind }}\right) \quad \text { (point-quadric structure) }
\end{aligned}
$$

where $\mathrm{I}_{\text {ind }}$ denotes in each case the incidence relation induced by $R_{1}\left(\right.$ or $\left.R_{2}\right)$ in the obvious sense.

(4.10) Theorem. (1) $\mathcal{I}^{\prime}$ and $\mathcal{I}^{\prime \prime}$ are $2-(176,8,2)$ designs on which $G$ acts as a group of automorphisms. $\bar{G}$ induces naturally an isomorphism between $\mathcal{I}^{\prime}$ and $\mathcal{I}^{\prime \prime}$.

(2) $\mathcal{I}$ is a symmetric $2-(176,50,14)$ design on which $G$ acts as a group of automorphisms. $\bar{G}$ acts on $\mathcal{I}$ as a group of correlations interchanging points and quadrics.

(3) $\left(X^{\prime}, X^{\prime \prime}, X ; R_{1}\right)$ provides for a model of $G$. Higman's geometry defined in [17]. G acts on this model as a group of automorphisms, $\bar{G}$ acts on $\mathcal{I}$ as a group of correlations interchanging points and quadrics and leaving the set of conics invariant.

Proof. Since $G$ acts doubly-transitively on $X^{\prime}$ and $X^{\prime \prime}$ and transitively on $X$ we immediately obtain that $\mathcal{I}^{\prime}$ is a $2-\left(176, k^{\prime}, \lambda^{\prime}\right)$ design, that $\mathcal{I}^{\prime \prime}$ is a $2-\left(176, k^{\prime \prime}, \lambda^{\prime \prime}\right)$ design and that $\mathcal{I}$ is a $2-(176, k, \lambda)$ design. From (4.8) it follows that $k^{\prime}=k^{\prime \prime}=8$ and that $k=50$. The canonical equations for the design parameters now yield $\lambda^{\prime}=2=\lambda^{\prime \prime}$ and $\lambda=14$. It is clear from the definition that $G$ acts on $\mathcal{I}^{\prime}, \mathcal{I}^{\prime \prime}$ and $\mathcal{I}$ as a group of automorphisms. It follows also from (4.8) that $\bar{G}$ induces an isomorphism between $\mathcal{I}^{\prime}$ and $\mathcal{I}^{\prime \prime}$ and acts as a group of correlations (inducing a polarity). It is now straightforward to verify that the "axioms" of G. Higman's geometry are fulfilled, see [17]. (Note that the mapping $\Theta$ of (4.7) is intimately related to the "conic correspondence" required in G. Higman's property (vi) in an obvious way.)

The assertion of (4.10) is illustrated by the following diagram, following the conventions used in Section 3. 


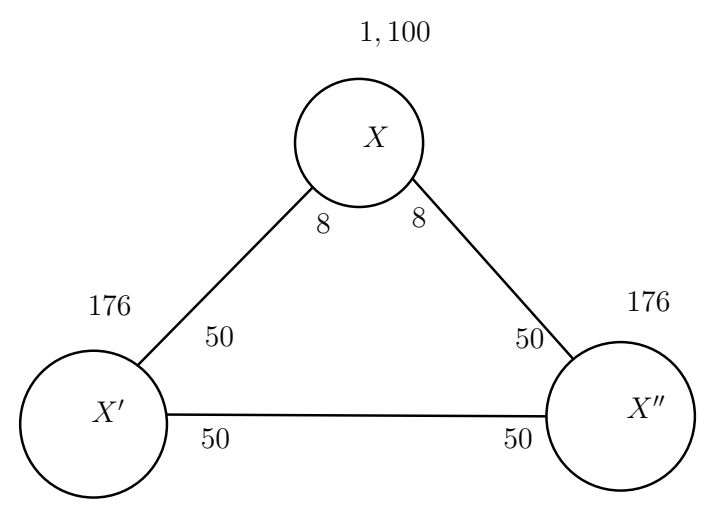

(4.11) Corollary.

The Higman-Sims group $G=$ HS is isomorphic to the automorphism group of G. Higman's geometry.

Proof. By (4.10) $G$ is isomorphic to a subgroup of the automorphism group of G. Higman's geometry. Comparison of group orders now gives the result.

Remark. It is plainly clear that - as a general principle of construction - the additive structure of a subquotient of a linear code left pointwise invariant by a group $G$ of code automorphisms may be used to define incidence structures admitting $G$ acting as a group of automorphisms.

\section{$5 \quad$ Subgroups of $\mathrm{G}=\mathrm{HS}$ given by the code $\mathrm{H}_{23}$}

It has been shown by Conway [7] and Curtis [8] that the major part of the maximal subgroups of the maximal subgroups of the Mathieu group $M_{24}$ may be described in terms of the binary Golay code of length 24. In this section we will show that the code $H_{23}$ serves for this purpose as well in the case of the Higman-Sims group.

We start with the following general concept.

\section{(5.1) Definition.}

Let a group $G$ act on a set $X$.

(1) A subgroup $U$ of $G$ is called an $X$-subgroup if and only if $U=G_{x}$ for some $x \in X$.

(2) The set of all $X$-subgroups of $G$ is denoted by $\operatorname{sub}_{X}(G)$. sub ${ }_{X}$ is a union of conjugacy classes of subgroups of $G$.

(3) $\operatorname{sub}_{X}(G)$ is partially ordered under inclusion. A subgroup $U$ of $G$ is called $X$ maximal if and only if $U \neq G$ and $U \leqslant V \in \operatorname{sub}_{X}(G)$ implies $V \in\{U, G\}$.

the set of all $X$-maximal subgroups of $G$ is denoted by $\max _{X}(G)$.

Note that $\max _{X}(G)=\emptyset$ if and only if $G$ acts trivially on $X$.

In the following we retain the notation of the previous sections. In particular $G=\mathrm{HS}$ denotes the Higman-Sims simple group and $F=\mathbb{F}_{2}$. We consider the action of $G$ on the FG-module $H_{23}$. 


\section{(5.2) Theorem.}

Every $\mathrm{H}_{23}$-maximal subgroup of $G$ is conjugate to exactly one subgroup in the following list.

(a) $G_{\alpha} \cong M_{22}$ of index 100 ;

(b) $G_{\{\alpha, \beta\}} \cong \mathrm{P} \Sigma \mathrm{L}(3,4)$ of index 1,100 where $\alpha$ and $\beta$ are joined in the Higman-Sims graph;

(c) $G_{\{\alpha, \gamma\}} \cong E_{16} \Sigma_{6}$ of index 3,850 where $\alpha$ and $\gamma$ are not joined in the Higman-Sims graph;

(d) $G_{x_{30}} \cong \Sigma_{8}$ of index 1,100 where $x_{30} \in W_{30}\left(H_{23}\right)$, a conic stabilizer in $G$. Higman's geometry;

(e) $G_{x_{36}} \cong 2^{6} \mathrm{GL}(3,2)$ of index 4,125 where $x_{36} \in W_{36}\left(H_{23}\right)$;

(f) $G_{x_{40}} \cong Z_{2} \times \operatorname{P\Gamma L}(2,9)$ of index 15,400 where $x_{40} \in 40_{1}$, the centralizer in $G$ of a fixed-point free involution;

(g') $G_{x_{34}^{\prime}} \cong M_{11}$ of index 5,600 where $x_{34}^{\prime} \in W_{34}\left(H_{22}^{\prime}\right)$;

$\left(g^{\prime \prime}\right) G_{x_{34}^{\prime \prime}} \cong M_{11}$ of index 5,600 where $x_{34}^{\prime \prime} \in W_{34}\left(H_{22}^{\prime \prime}\right)$;

( $\left.h^{\prime}\right) G_{x_{50}^{\prime}} \cong \operatorname{PSU}\left(3,5^{2}\right)$ of index 352 where $x_{50}^{\prime} \in X_{0}^{\prime}$;

$\left(h^{\prime \prime}\right) G_{x_{50}^{\prime \prime}} \cong \operatorname{PSU}\left(3,5^{2}\right)$ of index 352 where $x_{50}^{\prime \prime} \in X_{0}^{\prime \prime}$.

Proof. The assertion of the theorem follows essentially from (3.11), (3.20), (3.21) and its proofs. Some arguments of the omitted proof of (3.13) are also required concerning the orbits in $\Omega$ of the stabilizers $G_{x}, x \in H_{22}$. Since these arguments are elementary and tedious we omit the details.

Note that $\bar{G} \cong \operatorname{Aut}(G)$ fuses the conjugacy classes $\left(\mathrm{g}^{\prime}\right)$ and $\left(\mathrm{g}^{\prime \prime}\right)$, resp. $\left(\mathrm{h}^{\prime}\right)$ and $\left(\mathrm{h}^{\prime \prime}\right)$.

It can be shown that all $H_{23}$-maximal subgroups of $G=\mathrm{HS}$ are in fact maximal subgroups of $G$ with exception of the cases $\left(\mathrm{h}^{\prime}\right)$ and $\left(\mathrm{h}^{\prime \prime}\right)$. The $H_{23}$-maximal subgroups of $G$ of types $\left(\mathrm{h}^{\prime}\right)$ and $\left(\mathrm{h}^{\prime \prime}\right)$ are contained with index 2 in maximal subgroups isomorphic to $\mathrm{P} \Sigma \mathrm{U}\left(3,5^{2}\right)$ as easily follows from Section 4 . From (3.21) we know that these maximal subgroups are $H_{23} / H_{1}$-groups. More precisely we have the following result.

\section{(5.3) Theorem.}

Every $H_{23} / H_{1}$-maximal subgroup of $G$ is either $H_{23}$-maximal or a maximal subgroup of index 176 conjugate to a point-stabilizer or a quadric-stabilizer in G. Higman's geometry.

Proof. The assertion follows from (5.2), (3.20), (3.21) and Section 4, in particular Theorem (4.10). 
It is not difficult to show that the subgroups of types $(\mathrm{a}) \ldots\left(\mathrm{g}^{\prime \prime}\right)$ are in fact maximal subgroups of $G$. Of course, this follows from Magliveras [25] where reference is given to his unpublished dissertation [24]. We give an example for an independent direct argument.

\section{(5.4) Lemma.}

Let $x \in W_{30}\left(H_{23}\right)$. Then $G_{x} \cong \Sigma_{8}$ is a maximal subgroup of $G$.

Proof. Suppose $G_{x}<H \leqslant G$. Then $G_{x}$ has just 2 orbits in $\Omega$ : $\operatorname{supp}(x)=\Lambda_{8}(x)$ and $\Lambda_{6}(x)=\operatorname{supp}(x+\mathbb{1})$, see $(3.10)$. So we conclude that $H$ acts transitively on $\Omega$.

It follows from (3.10) that $\left(G_{x}\right)_{\beta}$ has orbits of length 8 and 14 in $\Delta(\beta)$ if $\beta \in \Lambda_{8}(x)$ and that $\left(G_{x}\right)_{\gamma}$ has orbits of length 16 and 6 in $\Delta(\gamma)$ if $\gamma \in \Lambda_{6}(x)$. Moreover, $\left(G_{x}\right)_{\gamma}$ contains a Sylow 7-subgroup of $G$ for $\gamma \in \Lambda_{6}(x)$. We easily conclude that $H_{\xi}$ acts primitively on $\Delta(\xi)$ for $\xi \in \Omega$. By a theorem of Wielandt [38, 31.1], the subgroup $H_{\xi}$ acts 2-transitively on $\Delta(\xi)$ (and it follows that $H_{\xi}$ has the orbits $\{\xi\}, \Delta(\xi)$ and $\Delta \circ \Delta(\xi)=\Delta_{2}(\xi)$ in $\Omega$ ) since $M_{22}$ has no proper subgroup acting doubly-transitively on 22 points. Hence $H=G$.

It is a result of Magliveras $[24,25]$ that $G$ has only two conjugacy classes of maximal subgroups which are not $H_{23} / H_{1}$-maximal:

(i) The centralizer of an involution with fixed-points (induced by an elation in $\operatorname{PSL}(3,4)$ $=M_{21}$ ), of index 5,775 with structure $2^{6} \Sigma_{5}$, acting intransitively on $\Omega$ with two orbits of lengths 20 and 80 .

(ii) The normalizer of the cyclic group generated by an element of order 5 whose centralizer in $G$ is of order 300, of index 36,960 and acting transitively on $\Omega$ with a system of imprimitivity of type $20^{5}$, in ATLAS notation: $5: 4 \times S_{5}$. see [1].

We recall that by Proposition (3.33) the groups in class (i) contain $H_{23}$-subgroups isomorphic to $\mathrm{GL}(2,3)$ with index 160.

It is easy to show that the groups in the class (i) are $H_{78}$-maximal subgroups of $G$. Note that $H_{79}$ is the inverse image under $v$ of $H_{1}=\langle\mathbb{1}\rangle$ and that $H_{22}=\operatorname{Im} v \leqslant H_{79}$, hence a forteriori every intransitive subgroup of $G$ fixes a vector in $H_{79} \backslash H_{1}$.

\section{References}

[1] ATLAS of finite groups by J. H. Conway, R.T. Curtis, S.P. Norton, R.A. Parker, R.A. Wilson. with computational assistance from J.G. Thackray. Clarendon Press, Oxford 1985.

[2] An ATLAS of Brauer Characters by C. Jansen, K. Lux, R. Parker, R. Wilson. Clarendon Press, Oxford 1995.

[3] Brauer, R.D. : On the connections between the ordinary and the modular characters of groups of finite order. Ann. Math. 42, 926-935 (1941).

[4] Brouwer, A. E. and van Eijl, C.A. : On the p-rank of the adjacency matrices of strongly regular graphs. J. Alg. Combinatorics, vol. 1, 329-346 (1992). 
[5] Brouwer, A. E. "Bounds on linear Codes" in : V.S. Pless and W.C. Huffman (Eds.) Handbook of Coding Theory, pp 295-461, Elsevier 1998.

[6] Calderbank, A.R. and Wales, D.B. : A global code invariant under the Higman-Sims group. J. Algebra 75, 233-260 (1982).

[7] Conway, J.H. : Three lectures on Exceptional Groups. In : Finite Simple Groups (Powell and Higman editors), Academic Press 1971.

[8] Curtis, R.T. : The maximal subgroups of $M_{24}$. Math. Proc. Camb. Phil. Soc. 81, 185-192 (1977).

[9] Dembowski, P. : Finite Geometries, Springer 1968.

[10] GAP4. The GAP Group, GAP - Groups, Algorithms, and Programming, Version 4.6.4; 2013. http://www.gap-system.org

[11] Griess, R.L. : A sufficient condition for a finite group of even order to have a nontrivial Schur multiplicator. Notices A.M.S. 17, 644 (1970).

[12] Haemers, Willem H., Peeters, René and van Ruckevorsel, Jeroem M. : Binary codes of strongly regular graphs. Designs Codes and Cryptography 17, 187-209 (1999).

[13] Hafner, P. R. : On the graphs of Hoffman-Singleton and Higman-Sims, Electronic Journal of Combinatorics 11 (2004) \# R77

[14] Higman, D.G. : Primitive rank 3 groups with a prime subdegree. Math. Z. 91, 70-86 (1966).

[15] Higman, D.G. : Intersection Matrices for finite permutation groups. J. Algebra 6, 22-42 (1967).

[16] Higman, D.G. and Sims, C.C. : A simple group of order 44,352,000. Math. Z. 105, 110-113 (1968).

[17] Higman, G. : On the simple group of D.G. Higman and C.C. Sims. Illinois J. Math. 13, 74-80 (1969).

[18] Huppert, B. : Darstellungstheorie endlicher Gruppen III. Vorlesungsausarbeitung, Universität Mainz 1974.

[19] James, G.D. : The modular characters of the Mathieu groups. J. Algebra 27, 57-111 (1973).

[20] Knapp, W. and Schmid, P. : Codes with prescribed permutation group. J. Algebra 67, 415-435 (1980).

[21] Loebich, N. : Rang 3-Gruppen mit dreiecksfreiem Graphen. Wissenschaftliche Arbeit, Universität Tübingen, 1981.

[22] Lüneburg, H. : Transitive Erweiterungen endlicher Permutationsgruppen. Lecture Notes in Mathematics 84, Springer 1969.

[23] MacWilliams, F.J. and Sloane, N.J.A. : The Theory of Error-Correcting Codes, I and II. North-Holland 1977.

[24] Magliveras, S.S. : The subgroup structure of the Higman-Sims simple group. Ph.D. Thesis, Birmingham University, England 1970. 
[25] Magliveras, S.S. : The subgroup structure of the Higman-Sims simple group. Bulletin A.M.S. 77, 535-539 (1971).

[26] Mazet, P. : Sur le mutiplicateur de Schur du group de Mathieu M22. C.R. Acad. Sci. Paris Sér. A 289, 659-661 (1979).

[27] McKay, J. and Wales, D. : The multiplier of the Higman-Sims simple group. Bull. London Math. Soc. 3, 283-285 (1971).

[28] Pless, V. : Power Moment Identities on Weight distributions in Error Correcting Codes. Information and Control 6, 147-152 (1963).

[29] Rudvalis, A. : Characters of the Covering Group of the Higman-Sims Group. J. Algebra 33, 135-143 (1975).

[30] Sims, C.C. : On the isomorphism of two groups of order 44,352,000. In: Theory of Finite Groups, a Symposium, ed. by R. Brauer and C. Sah. W.A. Benjamin 1969.

[31] Sims, C.C. : Computational Methods in the study of permutation groups. In: Computational problems in abstract algebra, John Leech (Editor). Pergamon Press 1970.

[32] Smith, M.S. : A Combinatorial Configuration Associated with the Higman-Sims Simple Group. J. Algebra 41, 175-195 (1976).

[33] Smith, M.S. : On the isomorphism of two groups of order 44,352,000. J. Algebra 41, 172-174 (1976).

[34] Stein, W.A. et al. : Sage Mathematics Software (Version 6.4.1), The Sage Development Team, 2014. http://www. sagemath.org

[35] Thackray, J. : private Communication, 1980.

[36] Thompson, J. G. : Vertices and Sources. J. Algebra 6, 1-6 (1967).

[37] Tonchev, V.D. : Binary codes derived from the Hoffman-Singleton and Higman-Sims graphs. IEEE Transactions of Information Theory 43 (3), 1021-1025 (1997).

[38] Wielandt, H. : Finite Permutation Groups. Academic Press 1964.

[39] Witt, E. : Die 5-fach transitiven Gruppen von Mathieu. Abh. Math. Sem. Univ. Hamb. 12, 256-264 (1938).

[40] Witt, E. : Über Steinersche Systeme. Abh. Math. Sem. Univ. Hamb. 12, 265-275 (1938). 